Stochastic density ratio estimation and its application to feature selection 



\title{
Stochastic density ratio estimation and its application to feature selection ${ }^{1}$
}

\author{
Ígor Assis Braga
}

Advisor: Profa. Dra. Maria Carolina Monard Co-advisor: Prof. Dr. Vladimir Naumovich Vapnik

Doctoral dissertation submitted to the Instituto de Ciências Matemáticas e de Computação - ICMC-USP, in partial fulfillment of the requirements for the degree of the Doctorate Program in Computer Science and Computational Mathematics. FINAL VERSION.

\section{USP - São Carlos}

December 2014

\footnotetext{
${ }^{1}$ This work is supported by grant \#2009/17773-7, São Paulo Research Foundation (FAPESP).
} 
Ficha catalográfica elaborada pela Biblioteca Prof. Achille Bassi e Seção Técnica de Informática, ICMC/USP, com os dados fornecidos pelo(a) autor(a)

Braga, Ígor Assis
Stochastic density ratio estimation and its
application to feature selection / Ígor Assis
Braga; orientadora Maria Carolina Monard; co-
orientador Vladimir Vapnik. -- São Carlos, 2014.
139 p.
Tese (Doutorado - Programa de Pós-Graduação em
Ciências de Computação e Matemática Computacional) --
Instituto de Ciências Matemáticas e de Computação,
Universidade de São Paulo, 2014.
1. density ratio estimation. 2. mutual
information estimation. 3. feature selection. 4.
SVM parameter selection. 5. RLS parameter
selection. I. Monard, Maria Carolina, orient. II.
Vapnik, Vladimir, co-orient. III. Título.




\title{
Estimação estocástica da razão de densidades e sua aplicação em seleção de atributos ${ }^{1}$
}

\author{
Ígor Assis Braga
}

Orientadora: Profa. Dra. Maria Carolina Monard Coorientador: Prof. Dr. Vladimir Naumovich Vapnik

Tese apresentada ao Instituto de Ciências Matemáticas e de Computação - ICMC-USP, como parte dos requisitos para obtenção do título de Doutor em Ciências - Ciências de Computação e Matemática Computacional. VERSÃO REVISADA.

\footnotetext{
${ }^{1}$ Apoio processo no 2009/17773-7, Fundação de Amparo à Pesquisa do Estado de São Paulo (FAPESP).
} 



\section{Acknowledgments}

This work is the product of a series of fortunate events, which granted me the support of the right people in the right places at the right time.

To begin with, the constant supervision of Prof. Maria Carolina Monard, who made every effort so that this work could take place, guiding me through the perils of graduate school.

The co-supervision of Prof. Vladimir Vapnik, to whom I am indebted for the immersion in the science of Empirical Inference during my research internship abroad and for having opened my eyes to the multitude of things I still do not know.

The support of the Sao Paulo Research Foundation - FAPESP — , which not only provided for me and my research in the graduate years, but was also sensible enough in dealing with bureaucratic procedures related to my research internship, in which case FAPESP scientific director, Prof. Carlos Henrique de Brito Cruz, gave special consideration.

The support of Rauf Izmailov and the suggestions of anonymous reviewers, who have contributed to enriched this work. The effort of ICMC-USP faculty and administrative staff, which makes possible our graduate program. The promptness of the staff at NEC Labs, where my research internship took place, specially when it came from Dr. Hans Peter Graf and Karen Smith.

The companionship of my friends in the Laboratory of Computational Intelligence LABIC - , specially of those who undertook the responsibility of running the lab infrastructure and of those who shared their good sense of humor.

The supervision, in the undergraduate years, of Prof. Marcelo Ladeira, who introduced me to machine learning and advised me to pursue the supervision of Prof. Carolina Monard at ICMC-USP.

The union to my beloved wife, who continuously stabilizes and propels me, and to whom I dedicate my life.

My birth to a family of wonderful parents and sister. Dad, Mom, this work is dedicated to you, to your efforts, and to the achievement of what you dreamed for your cub. 



\section{Agradecimentos}

Este trabalho é o produto de uma série de eventos afortunados, os quais me concederam o apoio das pessoas certas nos lugares certos e nos momentos certos.

Começando pela orientação sempre presente da Profa. Maria Carolina Monard, que não mediu esforços para fazer este trabalho acontecer, guiando-me pelos caminhos difíceis do mestrado e do doutorado.

A coorientação do Prof. Vladimir Vapnik, a quem eu devo pela imersão na ciência da Inferência Empírica durante o estágio de pesquisa no exterior e por ter me abrido os olhos para o quanto eu ainda não sei.

O apoio da Fundação de Amparo à Pesquisa do Estado de São Paulo — FAPESP — , tanto pela bolsa de doutorado que sustentou a mim e a minha pesquisa, quanto pela sensibilidade nos trâmites do estágio de pesquisa no exterior, contando nesse caso com atenção especial de seu diretor científico, Prof. Carlos Henrique de Brito Cruz.

O apoio de Rauf Izmailov e as sugestões dos diversos revisores anônimos, que contribuiram para enriquecer este trabalho. O esforço dos professores e servidores que faz a pós-graduação do ICMC-USP acontecer. A presteza da equipe da NEC Labs, onde realizei o estágio de pesquisa no exterior, em especial a do Dr. Hans Peter Graf e a de Karen Smith.

O convívio dos amigos do Laboratório de Inteligência Computacional - LABIC - , especialmente dos que se prontificaram a manter a infraestrutura do laboratório e dos que compartilharam seu bom humor.

A orientação, ainda na graduação, do Prof. Marcelo Ladeira, que me introduziu ao aprendizado de máquina e me aconselhou a buscar a orientação da Profa. Carolina Monard na pós-graduação do ICMC-USP.

A união com Meu Bem, que me estabiliza e me impulsiona continuamente, e a quem eu dedico a minha vida.

O meu nascimento de uma família de pais e irmã maravilhosos. Pai, Mãe, este trabalho é dedicado a vocês, aos seus esforços e à realização dos seus sonhos para a sua cria. 



\section{Abstract}

The estimation of the ratio of two probability densities is an important statistical tool in supervised machine learning. In this work, we introduce new methods of density ratio estimation based on the solution of a multidimensional integral equation involving cumulative distribution functions. The resulting methods use the novel $V$-matrix, a concept that does not appear in previous density ratio estimation methods. Experiments demonstrate the good potential of this new approach against previous methods.

Mutual Information - MI - estimation is a key component in feature selection and essentially depends on density ratio estimation. Using one of the methods of density ratio estimation proposed in this work, we derive a new estimator - VMI - and compare it experimentally to previously proposed MI estimators. Experiments conducted solely on mutual information estimation show that VMI compares favorably to previous estimators. Experiments applying MI estimation to feature selection in classification tasks evidence that better MI estimation leads to better feature selection performance.

Parameter selection greatly impacts the classification accuracy of the kernel-based Support Vector Machines - SVM. However, this step is often overlooked in experimental comparisons, for it is time consuming and requires familiarity with the inner workings of SVM. In this work, we propose procedures for SVM parameter selection which are economic in their running time. In addition, we propose the use of a non-linear kernel function - the min kernel - that can be applied to both low- and high-dimensional cases without adding another parameter to the selection process. The combination of the proposed parameter selection procedures and the min kernel yields a convenient way of economically extracting good classification performance from SVM.

The Regularized Least Squares - RLS - regression method is another kernel method that depends on proper selection of its parameters. When training data is scarce, traditional parameter selection often leads to poor regression estimation. In order to mitigate this issue, we explore a kernel that is less susceptible to overfitting - the additive INK-splines kernel. Then, we consider alternative parameter selection methods to cross-validation that have been shown to perform well for other regression methods. Experiments conducted on real-world datasets show that the additive INK-splines kernel outperforms both the RBF and the previously proposed multiplicative INK-splines kernel. They also show that the alternative parameter selection procedures fail to consistently improve performance. Still, we find that the Finite Prediction Error method with the additive INK-splines kernel performs comparably to cross-validation. 



\section{Resumo}

A estimação da razão entre duas densidades de probabilidade é uma importante ferramenta no aprendizado de máquina supervisionado. Neste trabalho, novos métodos de estimação da razão de densidades são propostos baseados na solução de uma equação integral multidimensional. Os métodos resultantes usam o conceito de matriz- $V$, o qual não aparece em métodos anteriores de estimação da razão de densidades. Experimentos demonstram o bom potencial da nova abordagem com relação a métodos anteriores.

A estimação da Informação Mútua - IM — é um componente importante em seleção de atributos e depende essencialmente da estimação da razão de densidades. Usando o método de estimação da razão de densidades proposto neste trabalho, um novo estimador - VMI — é proposto e comparado experimentalmente a estimadores de IM anteriores. Experimentos conduzidos na estimação de IM mostram que VMI atinge melhor desempenho na estimação do que métodos anteriores. Experimentos que aplicam estimação de IM em seleção de atributos para classificação evidenciam que uma melhor estimação de IM leva as melhorias na seleção de atributos.

A tarefa de seleção de parâmetros impacta fortemente o classificador baseado em kernel Support Vector Machines - SVM. Contudo, esse passo é frequentemente deixado de lado em avaliações experimentais, pois costuma consumir tempo computacional e requerer familiaridade com as engrenagens de SVM. Neste trabalho, procedimentos de seleção de parâmetros para SVM são propostos de tal forma a serem econômicos em gasto de tempo computacional. Além disso, o uso de um kernel não linear - o chamado kernel min - é proposto de tal forma que possa ser aplicado a casos de baixa e alta dimensionalidade e sem adicionar um outro parâmetro a ser selecionado. A combinação dos procedimentos de seleção de parâmetros propostos com o kernel min produz uma maneira conveniente de se extrair economicamente um classificador SVM com boa performance.

O método de regressão Regularized Least Squares - RLS - é um outro método baseado em kernel que depende de uma seleção de parâmetros adequada. Quando dados de treinamento são escassos, uma seleção de parâmetros tradicional em RLS frequentemente leva a uma estimação ruim da função de regressão. Para aliviar esse problema, é explorado neste trabalho um kernel menos suscetível a superajuste - o kernel INK-splines aditivo. Após, são explorados métodos de seleção de parâmetros alternativos à validação cruzada e que obtiveram bom desempenho em outros métodos de regressão. Experimentos conduzidos em conjuntos de dados reais mostram que o kernel INK-splines aditivo tem desempenho superior ao kernel RBF e ao kernel INK-splines multiplicativo previamente proposto. Os experimentos também mostram que os procedimentos alternativos de seleção de parâmetros considerados não melhoram consistentemente o desempenho. Ainda assim, o método Finite Prediction Error com o kernel INK-splines aditivo possui desempenho comparável à validação cruzada. 



\section{List of Acronyms}

CV Cross-Validation

CDF Cumulative Distribution Function

DRE Density Ratio Estimation

ECDF Empirical Cumulative Distribution Function

FPE Finite Prediction Error

GCV Generalized Cross-Validation

INK Infinite Number of Knots

JMI Joint Mutual Information

KLIEP Kulback Leibler Importance Estimation Procedure

KMM Kernel Mean Matching

LOOCV Leave-One-Out Cross-Validation

LSIF Least Squares Importance Filtering

MI Mutual Information

MLMI Maximum Likelihood Mutual Information

NRMSE Normalized Root Mean Squared Error

PDF Probability Density Function

RBF Radial Basis Function

RKHS Reproducing Kernel Hilbert Space

RLS Regularized Least Squares

RMSE Root Mean Squared Error

SC Schwartz Criterion 
SMS Shibata's Model Selector

SVM Support Vector Machines

VC Vapnik-Chervonenkis 


\section{List of Algorithms}

4.1 Joint Mutual Information - JMI . . . . . . . . . . . . 70

4.2 Feature selection with VMI + JMI . . . . . . . . . . . 71

5.1 Selection of SVM parameter $C \ldots \ldots \ldots$ 



\section{List of Figures}

2.1 Examples of unidimensional CDFs . . . . . . . . . . . . . . 33

2.2 Cumulative distribution functions and its empirical distribution $\ldots \ldots 33$

2.3 Bregman divergence $\ldots \ldots \ldots \ldots \ldots \ldots \ldots \ldots \ldots \ldots$

3.1 Distance between distribution functions . . . . . . . . . . . . . . 49

3.2 PCA transformation of a data set $\ldots \ldots \ldots \ldots \ldots \ldots$

4.1 Plot of functions $\log (z)$ and $z \log (z) \ldots \ldots \ldots \ldots 6$

4.2 Mutual information and data samples divided into two classes . . . . . 69

5.1 Effect of parameter $C$ in linear classification $\ldots \ldots \ldots \ldots$. . . . 81

5.2 SVM decision function using Gaussian RBF kernels . . . . . . . . . . . 82

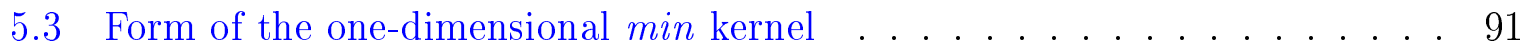

5.4 SVM classifier obtained using the $\min$ kernel . . . . . . . . . . . . . 91

5.5 Good and bad classification tasks for the min kernel . . . . . . . . . . . 92

5.6 Scatter plots for Linear versus RBF and INK-splines versus RBF . . . . 98

$5.7 \quad$ Min kernel versus Linear . . . . . . . . . . . . . . . . . . . . 100

$5.8 \quad$ Min kernel versus INK-splines . . . . . . . . . . . . . . . . . . . . 100

$5.9 \quad$ Min kernel versus RBF . . . . . . . . . . . . . . . . . . . . . 100

6.1 Best results for multiplicative INK-splines . . . . . . . . . . . . . . 119

6.2 Best results for additive INK-splines $\ldots \ldots \ldots \ldots \ldots \ldots$

A.1 Proposed SVM-RBF parameter selection procedure in WEKA . . . . . 130

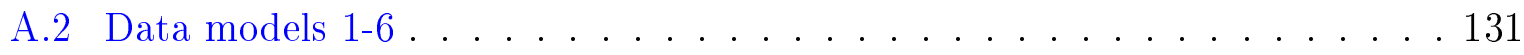

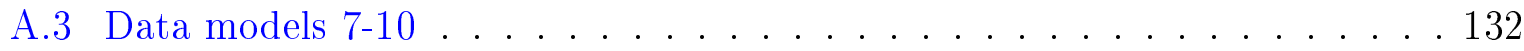





\section{List of Tables}

1.1 Symbol notation $\ldots \ldots \ldots \ldots \ldots \ldots \ldots \ldots \ldots \ldots \ldots$

3.1 Synthetic models . . . . . . . . . . . . . . . . . 60

3.2 Results of density ratio estimation based on the $V$-matrix concept versus

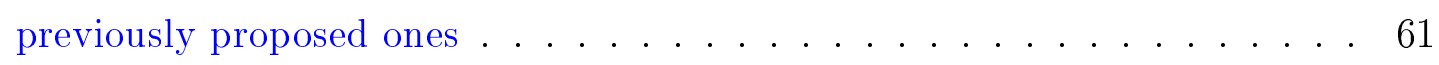

4.1 Synthetic models . . . . . . . . . . . . . . . . . 73

4.2 Mutual information estimation error of several methods . . . . . . . . . 74

4.3 Best and poorest results for MI estimation $\ldots \ldots \ldots \ldots \ldots \ldots$

4.4 Balanced error of an SVM classifier after feature selection according to

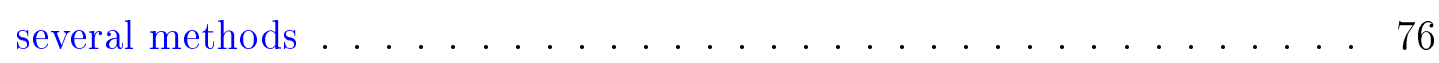

5.1 Classification datasets description $\ldots \ldots \ldots \ldots \ldots \ldots$

5.2 Balanced error evaluation measure $\ldots \ldots \ldots \ldots \ldots$

5.3 Linear kernel results in classification $\ldots \ldots \ldots \ldots \ldots$

5.4 INK-splines kernel results in classification . . . . . . . . . . . 96

$5.5 \quad$ Min kernel results in classification . . . . . . . . . . . . . . . 96

$5.6 \mathrm{RBF}$ inner squared distance distribution $\ldots \ldots \ldots \ldots \ldots$

5.7 RBF kernel results in classification . . . . . . . . . . . 98

5.8 Summary results for classifying high-dimensional datasets . . . . . . . . 101

5.9 Summary results for classifying text datasets . . . . . . . . . . . . 101

5.10 Summary results for classifying low-dimensional datasets . . . . . . . . 101

6.1 Classical complexity penalization $\ldots \ldots \ldots \ldots \ldots \ldots$

6.2 Regression datasets description $\ldots \ldots \ldots \ldots \ldots \ldots$

6.3 Experimental results for the best possible function and the one selected by leave-one-out across different kernel functions. . . . . . . . . . . 117 
6.4 Experimental results for statistical and heuristic parameter selection for the multiplicative INK-splines kernel . . . . . . . . . . . . . . 120

6.5 Experimental results for statistical and heuristic parameter selection for the additive INK-splines kernel . . . . . . . . . . . . . . . . . . 121

6.6 Summary of poor results for each INK-splines kernels . . . . . . . . . . . 122 


\section{Contents}

1 Introduction $\quad 23$

1.1 Density Ratio Estimation . . . . . . . . . . . . . . 23

1.2 Parameter Selection in Kernel Methods . . . . . . . . . . . . . . 25

1.3 Contributions and Organization . . . . . . . . . . . . 26

1.4 Notation and Terminology . . . . . . . . . . . . . . 28

2 Stochastic Theory of Density Ratio Estimation 31

2.1 Basic Concepts of Probability and Statistics . . . . . . . . . . . . 31

2.1.1 Cumulative Distribution Function . . . . . . . . . . . 32

2.1.2 Empirical Cumulative Distribution Function . . . . . . . . . 33

2.1.3 Probability Density Function . . . . . . . . . . . 35

2.2 Ill-Posed Problems . . . . . . . . . . . . . . . . 36

2.2.1 The Regularization Method .............. 37

2.2.2 The Solution of Density Estimation . . . . . . . . . . . . . 38

2.3 Stochastic Density Ratio Estimation . . . . . . . . . . . . . 38

2.4 Divergence-based Density Ratio Estimation . . . . . . . . . . . . . 40

2.4.1 Bregman Divergence .................. 40

2.4.2 Minimizing a Bregman Divergence from an Unknown Density Ratio 41

2.4.3 Previous Methods of Density Ratio Estimation . . . . . . . . . . . . 42

2.5 Final Remarks . . . . . . . . . . . . . . . . 43

3 Algorithms for Stochastic Density Ratio Estimation $\quad 45$

3.1 Distance between ECDF Functions . . . . . . . . . . . . . 46

$3.1 .1 \quad V$-Matrices $\ldots \ldots \ldots \ldots \ldots \ldots \ldots$ 
3.1 .2 More Advanced Distances . . . . . . . . . . . . . . . . 49

3.2 Regularizing Functional $\ldots \ldots \ldots \ldots \ldots \ldots$

3.2.1 Reproducing Kernel Hilbert Space and Its Norm . . . . . . . . . 51

3.2 .2 More Advanced Regularizers . . . . . . . . . . . . . . . . . 52

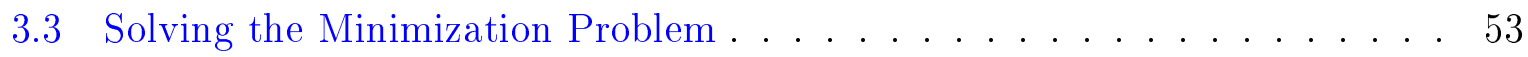

3.3 .1 Solution at Given Points (DRE-V) $\ldots \ldots \ldots \ldots \ldots$

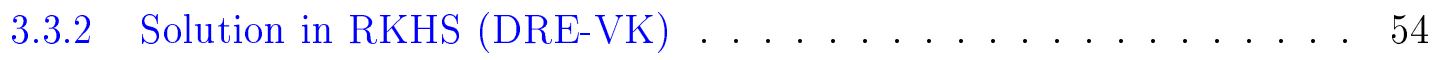

3.4 Selection of the Regularization Constant . . . . . . . . . . . 55

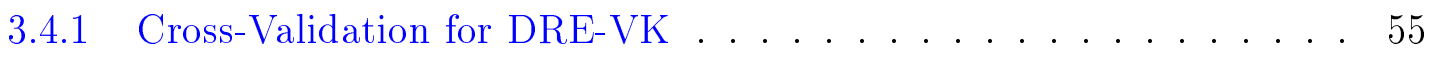

3.4 .2 Cross-Validation for DRE-V $\ldots \ldots \ldots \ldots \ldots$

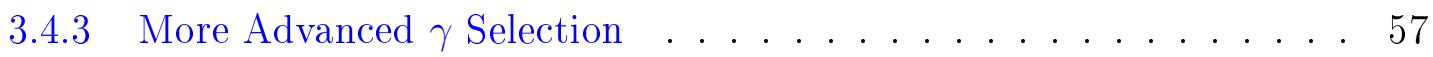

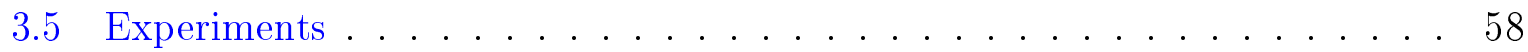

3.6 Final Remarks . . . . . . . . . . . . . . . . . . . . . . . 62

4 Mutual Information Estimation and Feature Selection 63

4.1 Mutual Information Estimation . . . . . . . . . . . . . . 64

4.1 .1 Definition of Mutual Information . . . . . . . . . . . 64

4.1 .2 Estimators of Mutual Information . . . . . . . . . . . 65

4.2 Feature Selection Based on Mutual Information . . . . . . . . . 67

4.2.1 Mutual Information and the Bayes Rule . . . . . . . . . 68

4.2.2 Heuristic Solutions to the Theoretical Problem . . . . . . . . . 68

4.3 Experiments . . . . . . . . . . . . . . . . . . 71

4.4 Final Remarks . . . . . . . . . . . . . . . . . 77

5 Economic Parameter Selection for SVM $\quad 79$

5.1 The Problem of Parameter Selection in SVM . . . . . . . . . . 80

5.1 .1 SVM and its Parameters . . . . . . . . . . 80

5.1 .2 Current Selection Strategies $\ldots \ldots \ldots$. . . . . . . . 82

5.2 Selecting the Generalization Parameter . . . . . . . . . . . 84

5.3 Selecting the Kernel Function $\ldots \ldots \ldots \ldots$. . . . . . 86

5.3 .1 Selecting the RBF Parameter . . . . . . . . . . 88 
5.3.2 Non-linear Parameterless Kernels . . . . . . . . . . . . . . 89

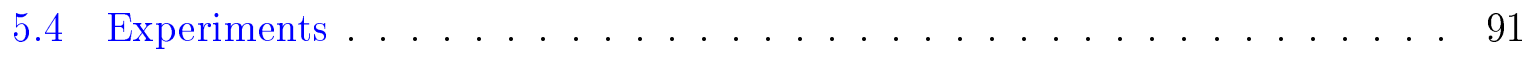

5.4 .1 Selection of $C \ldots \ldots \ldots \ldots$. . . . . . . . . . 94

5.4 .2 Selection of $\sigma \ldots \ldots \ldots \ldots \ldots$. . . . . . . . . . 97

5.4 .3 Performance of the Min Kernel . . . . . . . . . . . . . . 99

$5.5 \quad$ Final Remarks . . . . . . . . . . . . . . . . . 102

6 Statistical and Heuristic Parameter Selection in RLS 103

6.1 Overview of RLS . . . . . . . . . . . . . . . . . 104

6.1.1 Solving the RLS Minimization Problem . . . . . . . . . . . . 104

6.1.2 Challenges in RLS Parameter Selection . . . . . . . . . . . . . 106

6.2 Statistical Parameter Selection . . . . . . . . . . . . . 107

6.2.1 Leave-One-Out Cross-Validation . . . . . . . . . . . . . . 107

6.2.2 Complexity Penalization . . . . . . . . . . . . . 109

6.3 Heuristic Parameter Selection . . . . . . . . . . . . . . . 110

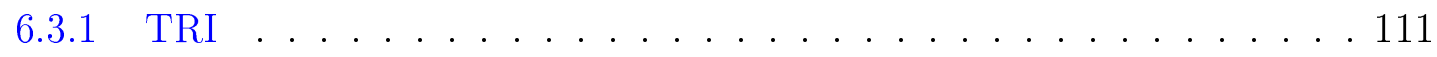

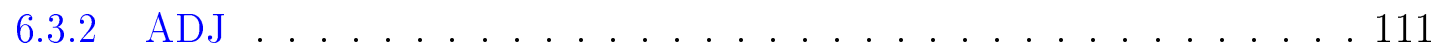

6.4 Choosing a Kernel Function for RLS . . . . . . . . . . . . . 112

6.5 Experiments . . . . . . . . . . . . . . . . . 114

6.5.1 Experimental Setup . . . . . . . . . . . . . . 114

6.5.2 Results and Discussion . . . . . . . . . . . . 116

6.6 Final Remarks . . . . . . . . . . . . . . . . . . 122

$\begin{array}{lll}7 & \text { Conclusion } & 125\end{array}$

$\begin{array}{lr}\text { A Appendix } & 129\end{array}$

A.1 RBF Parameter Selection in WEKA . . . . . . . . . . . 129

A.2 Synthetic Models for the Mutual Information Experiments . . . . . . . . 131

$\begin{array}{ll}\text { References } & 133\end{array}$ 



\section{Chapter 1}

\section{Introduction}

In this work, we deal with a relatively new statistical tool in machine learning: the estimation of the ratio of two probability densities, or density ratio estimation for short. In addition, as a side piece of research that gained its own traction, we tackle the task of parameter selection in learning algorithms based on kernel methods.

This introductory chapter is organized as follows. Sections 1.1 and 1.2 briefly motivate the problems of density ratio estimation and parameter selection in kernel methods, respectively. Section 1.3 summarizes our contributions to these problems and outlines the organization of this work. Finally, Section 1.4 provides notations and terminology used throughout the text.

\subsection{Density Ratio Estimation}

The problem of estimating a probability density function from data is widely recognized in unsupervised machine learning, where hidden structures in the data should be elucidated. In supervised learning, on the other hand, the estimation of statistical objects have been less prominent. The objects of interest in supervised learning are classifiers and regressions. However, this is not to say that classifiers and regressions do not depend on statistical objects, because they certainly do. Indeed, let us assume that $\boldsymbol{x} \in \boldsymbol{R}^{d}$ is a feature vector and $y$ is the target variable. Then, the optimal classifier depends on the conditional probability $P(y \mid \boldsymbol{x})$, whereas the regression function depends on the conditional density $p(y \mid \boldsymbol{x})$.

With the increasing adoption of supervised machine learning in real-world problems, it is high time to recollect some of its statistical links. Among the statistical inference problems that are useful to supervised learning, we find the estimation of the ratio of two probability densities $r(\boldsymbol{x})=\frac{p_{1}(\boldsymbol{x})}{p_{2}(\boldsymbol{x})}$. In what follows, we introduce situations where density ratio estimation naturally arises. 
Covariate-shift adaptation. Under the hood, most of the supervised learning algorithms perform the so-called Empirical Risk Minimization - ERM — principle, which selects a function $f_{n}^{*}$ from a given set of functions $\mathcal{F}$ that minimizes the average loss over a given set of training points $\left\{\left(\boldsymbol{x}_{1}, y_{1}\right), \ldots,\left(\boldsymbol{x}_{n}, y_{n}\right)\right\}$. Formally:

$$
f_{n}^{*}=\underset{f \in \mathcal{F}}{\arg \min } \frac{1}{n} \sum_{i=1}^{n} \operatorname{Loss}\left(f\left(\boldsymbol{x}_{i}\right), y_{i}\right)
$$

Assuming that each $\left(\boldsymbol{x}_{i}, y_{i}\right)$ is independently and identically distributed (i.i.d) according to a distribution $P_{\boldsymbol{x}, y}$ and the set of functions $\mathcal{F}$ has limited capacity (e.g VC-dimension), with increasing $n$, the average loss of $f_{n}^{*}$ converges with probability 1 to the smallest possible expected loss of a function $f \in \mathcal{F}$ (Vapnik, 1998):

$$
\frac{1}{n} \sum_{i=1}^{n} \operatorname{Loss}\left(f_{n}^{*}\left(\boldsymbol{x}_{i}\right), y_{i}\right) \stackrel{n \rightarrow \infty}{\longrightarrow} \min _{f \in \mathcal{F}} \int \operatorname{Loss}(f(\boldsymbol{x}), y) d P_{\boldsymbol{x}, y} .
$$

This key result of Statistical Learning Theory justifies the use of the ERM principle when the obtained function is to be applied to points sampled from the same distribution as the training points.

Now, what happens if, in the real-world, test and training features (the so-called covariates) follow different distributions $P_{\boldsymbol{x}}^{1}$ and $P_{\boldsymbol{x}}^{2}$, respectively? In other words, if the distribution of $\boldsymbol{x}$ changes across training and test sets while $P(y \mid \boldsymbol{x})$ or $p(y \mid \boldsymbol{x})$ is kept intact, should we still use the ERM principle as stated in the previous paragraph? The short answer is no. By doing so, we violate the assumptions that guarantee convergence to the smallest possible expected loss in $\mathcal{F}$ according to distribution $P_{\boldsymbol{x}, y}^{1}$.

Fortunately, there is hope. Let us suppose that the distributions $P_{\boldsymbol{x}}^{1}$ and $P_{\boldsymbol{x}}^{2}$ admit densities $p_{1}$ and $p_{2}$ such that $p_{1}(\boldsymbol{x})>0$ implies $p_{2}(\boldsymbol{x})>0$. Then, the following modified ERM principle

$$
f_{n}^{*}=\underset{f \in \mathcal{F}}{\arg \min } \frac{1}{n} \sum_{i=1}^{n} \frac{p_{1}\left(\boldsymbol{x}_{i}\right)}{p_{2}\left(\boldsymbol{x}_{i}\right)} \operatorname{Loss}\left(f\left(\boldsymbol{x}_{i}\right), y_{i}\right)
$$

guarantees the desired convergence on the test set (Sugiyama et al., 2011):

$$
\frac{1}{n} \sum_{i=1}^{n} \frac{p_{1}\left(\boldsymbol{x}_{i}\right)}{p_{2}\left(\boldsymbol{x}_{i}\right)} \operatorname{Loss}\left(f_{n}^{*}\left(\boldsymbol{x}_{i}\right), y_{i}\right) \stackrel{n \rightarrow \infty}{\longrightarrow} \min _{f \in \mathcal{F}} \int \operatorname{Loss}(f(\boldsymbol{x}), y) d P_{\boldsymbol{x}, y}^{1}
$$

Mutual information estimation and feature selection. Irrelevant features may degrade the performance of a learning algorithm. The problem of feature selection asks for criteria to tell apart relevant and irrelevant features. The information theoretic concept of mutual information is one of these criteria. 
In one of its forms, mutual information is calculated as

$$
I(\boldsymbol{X}, Y)=\int_{Y} \int_{\boldsymbol{X}} p(\boldsymbol{x}, y) \log \frac{p(\boldsymbol{x}, y)}{p(\boldsymbol{x}) p(y)} d \boldsymbol{x} d y
$$

The estimation of the value $I(\boldsymbol{X}, Y)$ depends on the density ratio $r(\boldsymbol{x}, y)=\frac{p(\boldsymbol{x}, y)}{p(\boldsymbol{x}) p(y)}$. Intuitively, mutual information is a way of comparing the densities $p(\boldsymbol{x}, y)$ and $p(\boldsymbol{x}) p(y)$, the former associated to the actual distribution of the data and the later associated to the distribution that assumes independence between the feature vector $\boldsymbol{x}$ and the target variable $y$. The closer $r(\boldsymbol{x}, y)$ is to the unity value for pairs $(\boldsymbol{x}, y)$ distributed according to $P_{\boldsymbol{x}, y}$, the more the feature vector and the target variable are independent of each other, in which case $I(\boldsymbol{X}, Y)=0$.

In this section, we skip the details about mutual information and its connection to feature selection, as there is an entire chapter devoted to this topic.

Density ratios arise in other domains, like change-detection in time series (Sugiyama et al., 2011). Still, covariate-shift adaptation and mutual information estimation are arguably the most important situations where density ratios arise in supervised machine learning. As expected, in both cases the density ratio function is unknown and needs to be estimated using data sampled according to the distribution of the involved densities.

Several approaches have been proposed and studied for the direct solution of the density ratio estimation problem, that is, to estimate the density ratio without going through density estimation (Gretton et al., 2009; Nguyen et al., 2010; Sugiyama et al., 2011, and references therein). By avoiding taking the ratio of two estimated densities, we avoid a dangerous source of estimation error propagation.

\subsection{Parameter Selection in Kernel Methods}

In supervised learning, there is an essential trade-off between training set error and the capacity of the given set of functions $\mathcal{F}$ : one can always be minimized at the expense of the other. Learning algorithms usually make it possible to explore this dilemma through a set of parameters. To obtain the best performance in practice, one needs to investigate candidate solutions in this parameter space.

This research topic stemmed from the main research topic on density ratio estimation and feature selection. Eventually, we realized the initial results fitted a broader purpose, which led us to further investigate the topic.

This way, we investigate in this work the parameter selection stage of Support Vector Machines - SVM - (Cortes and Vapnik, 1995) in classification and the Regularized Least Squares - RLS - method (Wahba, 1990; Girosi, 1997; Rifkin, 2006) in regression. 
Both learning algorithms belong to the category of kernel methods.

In general, SVM have two parameters to be selected: the generalization parameter $C$ and the kernel function $k$. The current practice in choosing these parameters leaves few alternatives. One either

- spends a lot of computational time using a comprehensive set of candidates that includes the best ones; or

- resorts to default parameters of the implementation of choice and risks achieving poor classification results.

Both alternatives are obviously unattractive. In order to avoid having to choose between these two alternatives in the feature selection experiments present in this work, it was important to investigate new alternatives that explore the gap between these two standard choices.

The RLS method has also two parameters: the regularizing constant $\gamma$ and the kernel function $k$. In the case of RLS, we are interested in the performance of parameter selection when training sets are small. The combination of the squared loss function and the regression task is a complicating factor for parameter selection using small training sets. Contrary to SVM, the computational time spent by parameter selection procedures is not an issue. What is relevant is the risk of overfitting due to the cross-validation parameter selection procedure. Therefore, it is important to investigate alternative candidate evaluation procedures. Another important trait of the parameter selection problem in RLS is that we face a similar problem for selecting parameters of the density ratio estimation methods proposed in this work.

\subsection{Contributions and Organization}

In this section, we provide a glimpse into the contributions that lie in the chapters ahead. We return to discussing our contributions at the end of each chapter and in the last chapter, where we also provide indications for future research.

The contributions in this work can be grouped into three categories:

1. Density ratio estimation;

2. Mutual information estimation and feature selection;

3. Parameter selection for SVM and RLS.

Regarding the first category, the approach to density ratio estimation taken in this work is based on unexplored ideas of searching the solution of a stochastic integral equation 
defining the ratio function. In this integral equation, we find the so-called empirical cumulative distribution functions. To the best of our knowledge, there is no attempt to use these functions in the literature of density ratio estimation. The proposed methods based on this approach outperform previous methods, with the advantage that their computational cost is no greater than previous methods. In this work, we cover this contribution in Chapters 2 and 3. This contribution also appears in (Vapnik et al., 2014): Vapnik, V., Braga, I., and Izmailov, R. (2014). A constructive setting for the problem of density ratio estimation. In SDM '14: Proceedings of the 2014 SIAM International Conference on Data Mining, pages 434-442.

Using one of the proposed methods of density ratio estimation, we develop a new mutual information estimation method, which, in turn, is employed in feature selection in classification tasks. Regarding mutual information estimation alone, the new estimator outperforms previous state-of-the-art methods. In feature selection, the resulting algorithm provides results that are comparable to the best ones, while outperforming the popular Relief-F (Kononenko, 1994) feature selection algorithm. In this work, we cover this contribution in Chapter 4. This contribution also appears in (Braga, 2013, 2014):

Braga, I. (2013). The constructive density-ratio approach to mutual information estimation: An experimental comparison. In KDMile '13: Proceedings of 2013 Symposium on Knowledge Discovery, Mining and Learning, pages 1-4.

Braga, I. (2014). A constructive density-ratio approach to mutual information estimation: experiments in feature selection. Journal of Information and Data Management, 5(1):134143.

Regarding parameter selection for SVM, we propose and evaluate easy-to-use and economic procedures that provide reasonable results. In addition, we propose a new kernel, namely the min kernel, which mixes the advantages of linear and non-linear parameterless kernels, making it suitable as a first choice kernel. In this work, we cover this contribution in Chapter 5. Some parts of this contribution also appears in (Braga et al., 2013):

Braga, I., do Carmo, L. P., Benatti, C. C., and Monard, M. C. (2013). A note on parameter selection for support vector machines. In MICAI '13: Proceedings of the 2013 Mexican International Conference on Artificial Intelligence, pages 233-244.

In RLS, we investigate several parameter selection methods that were shown to perform well for other regression methods. Unfortunately, no method is able to consistently outperform cross-validation, though we find situations where some alternative methods perform comparably well. Regarding the kernel function, we propose the use of the additive INK-splines kernel instead of RBF or the multiplicative INK-splines kernel. The proposed kernel function clearly outperforms the other ones in the small sample size regime. In this work, we cover this contribution in Chapter 6. This contribution also appears in (Braga and Monard, 2013, 2015): 
Braga, I. and Monard, M. C. (2013). Statistical and heuristic model selection in regularized least-squares. In BRACIS '13: Proceedings of the 2013 Brazilian Conference on Intelligent Systems, pages 231-236.

Braga, I. and Monard, M. C. (2015). Improving the kernel regularized least squares method for small-sample regression (in print). Neurocomputing.

\subsection{Notation and Terminology}

When the meaning of a particular symbol is not explicitly stated within a chapter, we assume the notation in Table 1.1. Subscripting and/or superscripting a symbol do not change its type.

Table 1.1: Symbol notation

\begin{tabular}{ll}
\hline Type & Notation \\
\hline Number & $a, b$ \\
Vector & $\boldsymbol{a}, \boldsymbol{b}$ \\
Constant & $c$ \\
Dimension & $d$ \\
Integer variable & $i, j, k, \ell, m, n$ \\
Real variable & $x, s, u, t, \alpha, \epsilon, \delta$ \\
Real vector & $\boldsymbol{x}, \boldsymbol{s}, \boldsymbol{u}, \boldsymbol{t}, \boldsymbol{\alpha}$ \\
Random variable & $X$ \\
Random vector & $\boldsymbol{X}$ \\
Set & $\mathcal{X}, \mathcal{Y}, \mathcal{F}$ \\
The real numbers & $\boldsymbol{R}$ \\
Function & $f$ \\
Cumulative distribution function $-\mathrm{CDF}$ & $F$ \\
Probability density function $-\mathrm{PDF}$ & $p$ \\
Indicator (step) function & $\theta$ \\
Ratio of densities function & $r$ \\
Functional & $L$ \\
Operator & $A$ \\
Inverse operator & $A^{-1}$ \\
Function value & $f(x)$ \\
Functional value & $L(f)$ \\
Image of a function through an operator & $A f$ \\
Probability measure (distribution) & $P$ \\
Probability measure of an event $E$ & $P\{E\}$ \\
Metric (distance) & $\rho$ \\
\hline
\end{tabular}

Throughout the text, we deal with different types of mappings between sets. They are traditionally distinguished in the following way. A mapping $f: \mathcal{X} \rightarrow \mathcal{Y}$ is said to be a function when $\mathcal{X}$ and $\mathcal{Y}$ are each a set of numbers (or vectors). A mapping $\mathcal{L}: \mathcal{F} \rightarrow \mathcal{Y}$ is said to be a functional when $\mathcal{F}$ is a set of functions and $\mathcal{Y}$ is a set of numbers. Lastly, a 
mapping $A: \mathcal{F} \rightarrow \mathcal{G}$ is said to be an operator when $\mathcal{F}$ and $\mathcal{G}$ are each a set of functions.

For compactness, we may denote a function value $f\left(x^{1}, \ldots, x^{d}\right)$ simply by $f(x)$. Likewise, we may employ the following notation for multiple integrals:

$$
\begin{gathered}
\int_{s}^{u} f(t) d t \equiv \int_{s^{1}}^{u^{1}} \ldots \int_{s^{d}}^{u^{d}} f\left(t^{1}, \ldots, t^{d}\right) d t^{1}, \ldots, d t^{d} \\
\int_{s}^{u} f(t) d g(t) \equiv \int_{s^{1}}^{u^{1}} \ldots \int_{s^{d}}^{u^{d}} f\left(t^{1}, \ldots, t^{d}\right) d g\left(t^{1}, \ldots, t^{d}\right) .
\end{gathered}
$$

Shall we distinguish single from multiple integrals, a warning note will be included in the text. Additionally, if we want to stress the multidimensional nature of the results, the multiple integral notation will be preferred.

Let us consider two numbers $a$ and $b$; we denote by $a \wedge b$ the function $\min (a, b)$; similarly, we denote by $a \vee b$ the function $\max (a, b)$. Now, consider two $d$-dimensional column vectors $\boldsymbol{a}=\left[a^{1}, \ldots, a^{d}\right]^{\top}$ and $\boldsymbol{b}=\left[b^{1}, \ldots, b^{d}\right]^{\top}$, and let $c$ be a constant. Then, the following expressions read:

- $\boldsymbol{a} \geq \boldsymbol{b}: a^{1} \geq b^{1}, \ldots, a^{d} \geq b^{d}$

- $\boldsymbol{a} \geq c: a^{1} \geq c, \ldots, a^{d} \geq c$

- $\boldsymbol{a} \wedge \boldsymbol{b}=\left[a^{1} \wedge b^{1}, \ldots, a^{d} \wedge b^{d}\right]^{\top}$

- $\boldsymbol{a} \vee \boldsymbol{b}=\left[a^{1} \vee b^{1}, \ldots, a^{d} \vee b^{d}\right]^{\top}$

We use different notations for $L^{p}$ norms and $p$-norms. The former is defined on function spaces, while the latter is defined on vector spaces. For real-valued functions whose domain is the real interval $[a, b]$, the standard $L^{p}$ norm, for a real $p \geq 1$, is denoted/defined by

$$
\|f\|_{L^{p}}=\left(\int_{a}^{b}|f(t)|^{p} d t\right)^{\frac{1}{p}} .
$$

On the other hand, a $p$-norm, for a real $p \geq 1$, is denoted/defined by

$$
\|\boldsymbol{x}\|_{p}=\left(\sum_{k=1}^{d}\left|x^{k}\right|^{p}\right)^{\frac{1}{p}}
$$

where $\boldsymbol{x}=\left[x^{1}, \ldots, x^{d}\right]^{\top}$. Norms other than the previously stated are also used in this work. In the event of their use, the norm notation $\|\cdot\|$ will be subscripted accordingly to highlight the difference. 


\section{Chapter 2}

\section{Stochastic Theory of Density Ratio Estimation}

In Chapter 1, we introduced and motivated the problem of estimating the ratio of two probability densities. In this chapter, we present the stochastic theory underlying this problem (Vapnik, 1998, Chapter 7) along with an overview of previously proposed methods of density ratio estimation (Sugiyama et al., 2012).

The computational methods for density ratio estimation proposed in Chapter 3 are based on the stochastic theory presented in this chapter. Central to this theory are two concepts covered in the following sections: the empirical cumulative distribution function - ECDF - and the regularization method for solving ill-posed problems.

This chapter is organized as follows. Section 2.1 presents fundamental concepts of probability theory and statistics, one of them being the ECDF. Section 2.2 explains the concept of ill-posed problems and presents the regularization method. Section 2.3 casts the problem of density ratio estimation as the problem of solving a stochastic ill-posed integral equation by means of the regularization method. Section 2.4 overviews prior work on density ratio estimation along the lines of Sugiyama et al. (2012), using the concept of Bregman divergences as a unifying framework. Section 2.5 concludes with historical remarks and a discussion as to why the theory presented in this chapter has the potential to advance the subject of density ratio estimation.

\subsection{Basic Concepts of Probability and Statistics}

In this section, we overview concepts that lie in the heart of probability theory and statistics. For the rigorous development of these concepts, the reader is referred to monographs on the subject (e.g Shiryayev, 1984).

We start with the probability space structure, which is a triple $(\mathcal{X}, \mathcal{E}, P)$ where: 
- $\mathcal{X}$ is a sample space, that is, each $x \in \mathcal{X}$ is a possible experimental outcome;

- $\mathcal{E}$ is a $\sigma$-algebra of subsets of $\mathcal{X}$, which means that each element of $\mathcal{E}$ is an event. Both the impossible event $\emptyset$ and the certain event $\mathcal{X}$ are elements of $\mathcal{E}$;

- $P: \mathcal{E} \mapsto[0,1]$ with $P\{\mathcal{X}\}=1$ is a probability measure.

Using this structure, it is possible to establish the main difference between probability theory and statistics (Shiryayev, 1984; Vapnik, 1998). The former concerns itself with the calculation of the probability of events $P\{E\}, E \in \mathcal{E}$, for a specified probability measure $P$. The latter, in its turn, is devoted to the elucidation of the probability measure $P$ (or some of its characteristics) through the observation of occurrence of random events $E \in \mathcal{E}$. The concepts presented in the following three sections reflect this dichotomy.

\subsubsection{Cumulative Distribution Function}

One of the basic concepts in probability theory is that of a random vector $\boldsymbol{X}=$ $\left(X^{1}, \ldots, X^{d}\right)$ and its cumulative distribution function $-\mathrm{CDF}-F$ :

$$
F\left(x^{1}, \ldots, x^{d}\right)=P\left\{\left(X^{1} \leq x^{1}\right) \cap \ldots \cap\left(X^{d} \leq x^{d}\right)\right\}
$$

From the definition of random vectors, which entails a particular probability space, such an $F$ always exists. If $F$ is known, then the probability measure $P$ of the underlying probability space is also known. That is, $F$ may be used to calculate the probability measure of each and every event regarding the random vector (Shiryayev, 1984, Section II.3). For instance, when $d=1$ and $-\infty \leq a<b<\infty$ :

$$
P\{a<X \leq b\}=F(b)-F(a) .
$$

The following properties apply to any CDF:

- $F$ is non-decreasing;

- $F(-\infty)=0$ and $F(\infty)=1$, where

$$
\begin{aligned}
F(-\infty) & =\lim _{x^{1} \downarrow-\infty} \ldots \lim _{x^{d} \downarrow-\infty} F\left(x^{1}, \ldots, x^{d}\right), \\
F(\infty) & =\lim _{x^{1} \uparrow \infty} \ldots \lim _{x^{d \uparrow} \infty} F\left(x^{1}, \ldots, x^{d}\right)
\end{aligned}
$$

- $F$ is right-continuous with left limits (cadlag).

Figure 2.1 depicts examples of unidimensional CDFs for varied probability measures. 


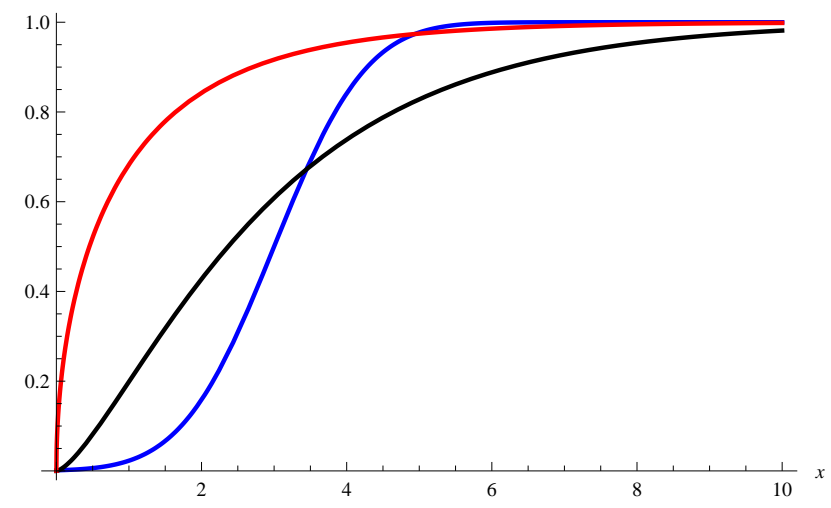

Figure 2.1: Examples of unidimensional CDFs

\subsubsection{Empirical Cumulative Distribution Function}

In statistics, a particular CDF is of fundamental importance, namely the empirical cumulative distribution function - ECDF. It is known that any $\operatorname{CDF} F\left(x^{1}, \ldots, x^{d}\right)$ is "well-approximated" by the ECDF $F_{\ell}\left(x^{1}, \ldots, x^{d}\right)$ constructed on the basis of an i.i.d sample distributed according to $F\left(x^{1}, \ldots, x^{d}\right)$ :

$$
\boldsymbol{x}_{1}, \ldots, \boldsymbol{x}_{\ell} \stackrel{i . i . d}{\sim} F(\boldsymbol{x})
$$

where $\boldsymbol{x}_{i}=\left(x_{i}^{1}, \ldots, x_{i}^{d}\right)$. Figure 2.2 depicts an example of an ECDF approximating a unidimensional CDF for both $\ell=50$ and $\ell=500$.

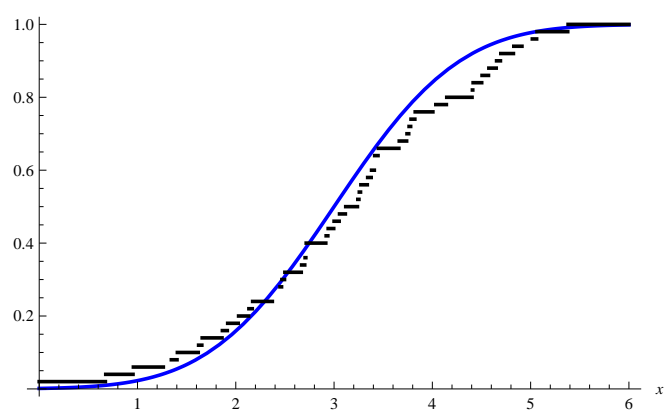

(a) 50-point ECDF

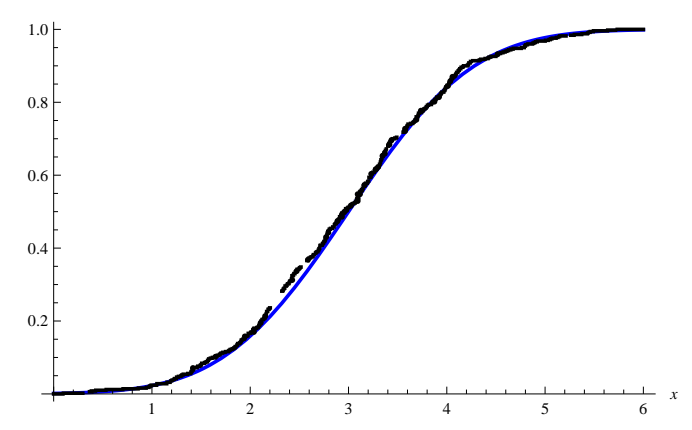

(b) 500-point ECDF

Figure 2.2: Cumulative distribution functions (in blue) of a univariate Gaussian distribution and its empirical distribution (ECDF, in black) based on points drawn from this distribution.

In essence, the ECDF value $F_{\ell}\left(x^{1}, \ldots, x^{d}\right)$ is the empirical estimator of the event $\left\{\left(X^{1} \leq x^{1}\right) \cap \ldots \cap\left(X^{d} \leq x^{d}\right)\right\}$. In other words, it is the fraction of points in $\boldsymbol{x}_{1}, \ldots, \boldsymbol{x}_{\ell}$ dominated by point $\boldsymbol{x}$ (i.e $\leq \boldsymbol{x}$, coordinate-wise). This relationship can be written as

$$
F_{\ell}\left(x^{1}, \ldots, x^{d}\right)=\frac{\#\left\{\boldsymbol{x}_{i} \leq \boldsymbol{x}\right\}}{\ell}=\frac{1}{\ell} \sum_{i=1}^{\ell} \prod_{k=1}^{d} \theta\left(x^{k}-x_{i}^{k}\right)
$$


where $\theta(u)$ is the step-function:

$$
\theta(u)=\left\{\begin{array}{ll}
1, & \text { if } u \geq 0 \\
0, & \text { otherwise }
\end{array} .\right.
$$

For simplicity, we refer to the multidimensional $F_{\ell}\left(x^{1}, \ldots, x^{d}\right)$ from now on as

$$
F_{\ell}(x)=\frac{1}{\ell} \sum_{i=1}^{\ell} \theta\left(x-x_{i}\right)
$$

We now explain what it means to say that the ECDF is a good approximation to the corresponding CDF. The formal investigation of this fact started in the 1930's with the Glivenko-Cantelli theorem, which states for any unidimensional $(d=1)$ CDF $F$ and any $\epsilon>0$ :

$$
P\left\{\sup _{x}\left|F_{\ell}(x)-F(x)\right|>\epsilon\right\} \stackrel{\ell \rightarrow \infty}{\longrightarrow} 0
$$

which means that the unidimensional ECDF $F_{\ell}$ converges in probability ${ }^{1}$ to the unidimensional CDF F. Still in the realm of unidimensional CDFs, the Dvoretzky-Kiefer-Wolfowitz inequality (Dvoretzky et al., 1956; Massart, 1990) establishes the fast convergence of $F_{\ell}(x)$ to $F(x)$, that is, for any natural $\ell$ and any $\epsilon>0$,

$$
P\left\{\sup _{x}\left|F_{\ell}(x)-F(x)\right|>\epsilon\right\} \leq 2 \exp \left(-2 \epsilon^{2} \ell\right)
$$

For multidimensional CDFs, $d>1$, fast convergence of $F_{\ell}(x)$ to $F(x)$ also takes place. As any CDF belongs to a set of monotonically increasing functions, and such a set has VC-dimension d, the Generalized Glivenko-Cantelli theorem (Vapnik, 1998, Section 4.9.3) can be applied, establishing convergence in probability of $F_{\ell}(x)$ to $F(x)$. A finite VC-dimension also guarantees the existence of the following inequality establishing exponential fast convergence:

$$
P\left\{\sup _{x_{1}} \ldots \sup _{x_{d}}\left|F_{\ell}\left(x_{1}, \ldots, x_{d}\right)-F\left(x_{1}, \ldots, x_{d}\right)\right|>\epsilon\right\} \leq 4 \exp \left(-a \epsilon^{2} \ell\right)
$$

where $a$ depends on $d$ and $\ell$.

One might wonder whether an estimator of the probability measure can be derived from the ECDF, in analogy to the actual probability measure arising from the CDF. In fact, the ECDF estimates the probability measure only partially, that is, only for a subset of the set of all events $\mathcal{E}$ (Vapnik, 1998, Chapter 2). For instance, when $d=1$, the ECDF estimates the probability measure only for events of the form $\{-\infty<X \leq x\}$, i.e $P\{X \leq x\}$.

\footnotetext{
${ }^{1}$ In fact, a stronger notion of convergence - almost sure convergence - also takes place.
} 
In fact, there cannot be an estimator for the probability of every possible event if the estimator is required to be an empirical estimator (which is independent of $P$ ). To see this, consider the following event parameterized by $\delta>0$ :

$$
E_{\delta}=\left\{x_{1}-\delta<X \leq x_{1}+\delta\right\} \cup \ldots \cup\left\{x_{\ell}-\delta<X \leq x_{\ell}+\delta\right\}
$$

For any $\ell$, it is possible to show (Vapnik, 1998, Section 2.2) there is always a $\delta^{*}$ so that, for all $\delta<\delta^{*}$, the estimate of the probability measure provided by the empirical estimator cannot approximate the actual probability measure of $E_{\delta}$.

\subsubsection{Probability Density Function}

If there is no single estimator independent of $P$ that completely estimates the probability measure, one may ask whether an estimator exists if we allow it to depend on $P$. Such an estimator exists for random vectors admitting a probability density function PDF — (Vapnik, 1998, Section 2.3).

The PDF associated to a random vector $\boldsymbol{X}=\left(X^{1}, \ldots, X^{d}\right)$ is a non-negative function $p$ defined as the derivative of the CDF of $\boldsymbol{X}$ :

$$
p\left(x^{1}, \ldots, x^{d}\right)=\frac{\partial F\left(x^{1}, \ldots, x^{d}\right)}{\partial x^{1} \ldots \partial x^{d}} .
$$

This derivative exists only when $F$ is an absolutely continuous function with respect to $x^{1}, \ldots, x^{d}$.

According to the definition of density function in Expression (2.4), the problem of density estimation from a given collection of data is the problem of solving the (multidimensional) integral equation

$$
\int_{-\infty}^{x} p(t) d t=F(x)
$$

when the cumulative distribution $F(x)$ is unknown but an i.i. $d$ sample $x_{1}, \ldots, x_{\ell} \sim F(x)$ is given. The constructive setting of this problem is to solve the equation in Expression (2.5) using the approximation in Expression (2.2) in place of $F(x)$. Remind that, according to Expression (2.3), $F_{\ell}$ converges uniformly to $F$ with probability 1.

It is known that solving a linear operator equation such as the integral equation in Expression (2.5) is an ill-posed problem: small deviations in the right-hand side $F$ may lead to large deviations of the solution $p$. As we cannot avoid an approximate right-hand side in this problem, any method for obtaining solutions $p_{\ell} \rightarrow p$ as $F_{\ell} \rightarrow F$ must consider the arguments of the theory of ill-posed problems, described in the next section.

For now, provided we obtain estimates $p_{\ell}$ of the density function such that $p_{\ell} \rightarrow p$ (in a suitable mode of convergence), then we obtain as well an estimator of the probability 
measure for any event $E$ (Vapnik, 1998, Section 2.3):

$$
P\{E\}=\int_{E} p_{\ell}(t) d t, \quad E \in \mathcal{E} .
$$

\subsection{Ill-Posed Problems}

A mathematical problem is considered to be well-posed when its solution:

- exists;

- is unique;

- is stable.

If one of the aforementioned requirements fails to hold, the problem is said to be ill-posed. In this work, we deal with the solution of ill-posed operator equations

$$
\text { Af }=T
$$

that satisfy the first two requirements but fail the third one.

While the meaning of the first two requirements is clear, the last requirement asks for further clarifications. In a few words, stability allows for small changes in the specification of a problem to cause only small changes in its original solution. As an example, let us consider solving for $f$ in Expression (2.6) using the solution $f_{\delta}$ of the approximating equation $A f=T_{\delta}$. If the problem of solving the equation in Expression (2.6) is stable, then the closer $T_{\delta}$ is to $T$, the closer the solution $f_{\delta}$ is to $f$.

Let $\mathcal{S}_{1}$ and $\mathcal{S}_{2}$ be two sets of functions and $A: \mathcal{S}_{1} \mapsto \mathcal{S}_{2}$ be an operator that establishes a bijective mapping between $\mathcal{S}_{1}$ and $\mathcal{S}_{2}$. For such an operator, the problem of solving the operator equation $A f=T$ for a given function $T \in \mathcal{S}_{2}$ is that of finding the function $f \in \mathcal{S}_{1}$ which is mapped to $T$ through $A$. Such a solution $f$ exists and is unique by the definition of bijective mappings.

Additionally, let the functions in $\mathcal{S}_{1}$ (resp. $\mathcal{S}_{2}$ ) belong to a metric space with metric $\rho_{1}$ (resp. $\rho_{2}$ ). In this case, A is said to be continuous when close functions in $\mathcal{S}_{1}$ (with respect to metric $\rho_{1}$ ) are mapped to close functions in $\mathcal{S}_{2}$ (with respect to metric $\rho_{2}$ ).

The question of whether the equation in Expression (2.6) is ill-posed for a continuous, bijective operator $\mathrm{A}$ is determined by the inverse operator $A^{-1}: \mathcal{S}_{2} \mapsto \mathcal{S}_{1}$. If $A^{-1}$ is continuous, the solution of the equation will be stable, since close functions $T_{1}, T_{2} \in \mathcal{S}_{2}$ with respect to metric $\rho_{2}$ will provide solutions $f_{1}=A^{-1} T_{1}$ and $f_{2}=A^{-1} T_{2}$ that are close to each other with respect to metric $\rho_{1}$ in $\mathcal{S}_{1}$. However, if $A^{-1}$ is discontinuous, the solution will be unstable, and hence the operator equation is ill-posed. 
Hereafter, we focus on continuous, bijective operators $A$ with discontinuous $A^{-1}$. Solving $A f=T$ in such cases is a hard problem whenever the right-hand side function $T$ and/or the operator $A$ are known only approximately.

\subsubsection{The Regularization Method}

In the 1960 's, methods were introduced to solve the case where approximations to the right-hand side of Expression (2.6) are given deterministically. Underlying these methods there is a regularizing (or stabilizing) functional $\Omega: \mathcal{S}_{1}^{*} \mapsto \boldsymbol{R}^{+}$satisfying the following requirements:

- the solution $f_{0}$ of the operator equation in Expression (2.6) is in $\mathcal{S}_{1}^{*}$;

- there exists $c_{0}, 0 \leq c_{0}<\infty$, for which $\Omega\left(f_{0}\right) \leq c_{0}$;

- for every $c$, the set $M_{c}=\left\{\hat{f} \in \mathcal{S}_{1}^{*}: \Omega(\hat{f}) \leq c\right\}$ is compact ${ }^{2}$.

The importance of the last requirement lies in the fact that for a continuous, bijective operator $A: M_{c} \mapsto \mathcal{S}_{2}$, the inverse operator $A^{-1}: \mathcal{S}_{2} \mapsto M_{c}$ is continuous, which provides a way of stabilizing the solution of $A f=T$.

All of the proposed regularization methods were shown to be equivalent. However, from the conceptual point of view, the method of Ivanov (1963) is clear regarding the stabilizing role that $\Omega(\hat{f})$ plays in solving ill-posed problems. Given approximations $T_{\delta}$ to the right hand side of Expression (2.6) such that

$$
\rho_{2}\left(T, T_{\delta}\right) \leq \delta
$$

the idea of Ivanov regularization is to find the solution of $A f=T$ by minimizing the following functional

$$
R\left(\hat{f}, T_{\delta}\right)=\rho_{2}\left(A \hat{f}, T_{\delta}\right), \quad \hat{f} \in \mathcal{S}_{1}^{*}
$$

under the constraint

$$
\Omega(\hat{f}) \leq c_{0}
$$

where $c_{0}$ is possibly unknown. The sequence of functions $f^{\delta}$ minimizing $R\left(\hat{f}, T_{\delta}\right)$ converges to the exact solution $f_{0}$ of the equation in Expression (2.6) as $\delta \rightarrow 0$.

For practical reasons, here and in the next chapter we consider the method of Tikhonov (1963). Given approximations $T_{\delta}$ as in Expression (2.7), the idea of Tikhonov regularization is to find the solution of $A f=T$ by minimizing the functional

$$
R_{\gamma}\left(\hat{f}, T_{\delta}\right)=\left[\rho_{2}\left(A \hat{f}, T_{\delta}\right)^{2}+\gamma \Omega(\hat{f})\right], \quad \hat{f} \in \mathcal{S}_{1}^{*},
$$

\footnotetext{
${ }^{2}$ See the Heine-Borel theorem.
} 
where $\gamma>0$ is a regularization parameter that depends on $\delta$. By requiring

$$
\begin{array}{r}
\gamma \rightarrow 0 \text { as } \delta \rightarrow 0 \\
\lim _{\delta \rightarrow 0} \frac{\delta^{2}}{\gamma}<\infty,
\end{array}
$$

it is possible to show that the sequence of functions $f_{\delta}^{\gamma}$ minimizing $R_{\gamma}\left(\hat{f}, T_{\delta}\right)$ converges to the exact solution $f_{0}$ of $A f=T$ as $\delta \rightarrow 0$. These requirements on $\gamma$ express the intuitive idea that $\gamma$ should not go to zero much faster than $\delta$ goes to zero.

\subsubsection{The Solution of Density Estimation}

The integral equation in Expression (2.5), here denoted by $A p=F$, is unstable (Vapnik, 1998, Section 1.12): small deviations in the right hand side $F$ may lead to large deviations of the solution $p$. This way, density estimation is an example of an ill-posed problem.

When the approximation to the right-hand side of the equation is stochastic in nature, the regularization method can also be applied (Vapnik and Stefanyuk, 1978; Vapnik, 1998). If $F_{\ell}$ converges in probability to $F$, it was shown that, if $\gamma_{\ell} \rightarrow 0$ as $\ell \rightarrow \infty$, then for arbitrary $\delta, \nu>0$ there is a $\ell>\ell_{0}(\delta, \nu)$ such that the following inequality holds for $p_{\ell}$ :

$$
P\left\{\rho_{1}\left(p, p_{\ell}\right)>\delta\right\} \leq P\left\{\rho_{2}\left(F, F_{\ell}\right)>\sqrt{\gamma_{\ell} \nu}\right\}
$$

For density estimation, in particular, the inequalities in Expressions (2.3) and (2.9) imply that

$$
P\left\{\rho_{1}\left(p_{0}, p_{\ell}\right)>\delta\right\} \leq c \exp \left(-c^{*} \gamma_{\ell} \nu \ell\right)
$$

Therefore, if

$$
\gamma_{\ell} \stackrel{\ell \rightarrow \infty}{\longrightarrow} 0 \text { and } \ell \gamma_{\ell} \stackrel{\ell \rightarrow \infty}{\longrightarrow} \infty
$$

the sequence $p_{\ell}$ converges in probability to the solution of $A p=F$.

\subsection{Stochastic Density Ratio Estimation}

In what follows, we consider the problem of estimating the ratio $r(x)$ of two probability densities $p_{1}(x)$ and $p_{2}(x)$ (assuming $p_{2}(x)>0$ whenever $p_{1}(x)>0$ ):

$$
r(x)=\frac{d F_{1}(x) / d x}{d F_{2}(x) / d x}=\frac{p_{1}(x)}{p_{2}(x)} .
$$

According to this definition, and analogously to density estimation, the problem of estimating the density ratio from data is the problem of solving the (multidimensional) 
integral equation

$$
\int_{-\infty}^{x} r(t) d F_{2}(t)=F_{1}(x)
$$

when the distribution functions $F_{1}(x)$ and $F_{2}(x)$ are unknown but samples

$$
\begin{aligned}
& x_{1}, \ldots, x_{\ell} \stackrel{i . i . d}{\sim} F_{1}(x) \text { and } \\
& x_{1}^{\prime}, \ldots, x_{n}^{\prime} \stackrel{i . i . d}{\sim} F_{2}(x)
\end{aligned}
$$

are given.

The constructive setting of this problem is to solve the equation in Expression (2.10) using the empirical distribution functions

$$
\begin{aligned}
& F_{1, \ell}(x)=\frac{1}{\ell} \sum_{i=1}^{\ell} \theta\left(x-x_{i}\right) \quad \text { and } \\
& F_{2, n}(x)=\frac{1}{n} \sum_{i=1}^{n} \theta\left(x-x_{i}^{\prime}\right)
\end{aligned}
$$

in place of the actual cumulative distributions $F_{1}(x)$ and $F_{2}(x)$.

Note that density ratio estimation leads to a more complicated ill-posed equation than density estimation, since the operator $A$, which depends on $F_{2}(x)$, is also approximately defined:

$$
\left(A_{n} r\right)(x)=\int_{\infty}^{x} r(t) d F_{2, n}(t)=\frac{1}{n} \sum_{i=1}^{n} r\left(x_{i}^{\prime}\right) \theta\left(x-x_{i}^{\prime}\right) .
$$

In this situation, in order to solve the equation in Expression (2.10), we use Tikhonov's regularization method (Tikhonov, 1963) and minimize the regularization functional

$$
r_{\ell, n}=\underset{r \in \mathcal{S}_{1}^{*}}{\arg \min }\left[\rho_{2}\left(A_{n} r, F_{1, \ell}\right)^{2}+\gamma_{\ell, n} \Omega(r)\right]
$$

Let the sequence of operators $A_{n}$ converge in probability to $A$ in the following operator norm:

$$
\left\|A-A_{n}\right\|=\sup _{r \in \mathcal{S}_{1}^{*}} \frac{\rho_{2}\left(A r, A_{n} r\right)}{\sqrt{\Omega(r)}} .
$$

Then, for arbitrary $\delta, C_{1}, C_{2}>0$, there exists $\gamma_{0}>0$ such that for any $\gamma_{\ell, n} \leq \gamma_{0}$ the following inequality holds for $r_{\ell, n}$ (Vapnik, 1998; Stefanyuk, 1986):

$$
\begin{aligned}
P\left\{\rho_{1}\left(r_{0}, r_{\ell, n}\right)>\delta\right\} \leq & P\left\{\left\|A-A_{n}\right\|>C_{2} \sqrt{\gamma_{\ell, n}}\right\} \\
& +P\left\{\rho_{2}\left(F_{1}, F_{1, \ell}\right)>C_{1} \sqrt{\gamma_{\ell, n}}\right\} .
\end{aligned}
$$

It was shown in (Vapnik, 1998, Section 7.2) that, if the solution of the equation in Expression (2.10) belongs to a set $\mathcal{S}_{1}^{*}$ of smooth functions (in fact, a set of continuous 
functions with bounded variation suffices), then

$$
\left\|A-A_{n}\right\| \leq \rho_{2}\left(F_{2}, F_{2, n}\right)
$$

For sufficiently large $\ell$ and $n$, the inequalities in Expressions (2.3), (2.12), and (2.13) imply that

$$
\begin{aligned}
P\left\{\rho_{1}\left(r_{0}, r_{\ell, n}\right)>\delta\right\} \leq & c_{2} \exp \left(-c_{2}^{*} n \gamma_{\ell, n} C_{2}^{2}\right) \\
& +c_{1} \exp \left(-c_{1}^{*} \ell \gamma_{\ell, n} C_{1}^{2}\right) .
\end{aligned}
$$

Thus, in a set $\mathcal{S}_{1}^{*}$ of smooth functions, the sequence $r_{\ell, n}$ in Expression (2.11) converges in probability to the solution of the equation in Expression (2.10) — the sought density ratio - when

$$
\gamma_{\ell, n} \stackrel{m \rightarrow \infty}{\longrightarrow} 0 \text { and } m \gamma_{\ell, n} \stackrel{m \rightarrow \infty}{\longrightarrow} \infty
$$

where $m=\min (\ell, n)$.

The arguments in this section make clear that density ratio estimation cannot be simply reduced to the estimation of the individual densities. Otherwise, we would risk constructing unregularized solutions, as the ratio of two estimated quantities may behave very badly - due to error propagation - even when the individual estimates are regularized.

\subsection{Divergence-based Density Ratio Estimation}

Several methods of density ratio estimation have been proposed in the past years. Rather than presenting each of them separately, here we follow Sugiyama et al. (2012) and present them as special cases of the problem of minimizing a Bregman divergence functional.

\subsubsection{Bregman Divergence}

The Bregman divergence concept (Bregman, 1967) can be seen as a generalized distance, or better yet, a directed distance. For simplicity, we start this explanation with the pointwise Bregman divergence. Let $f: \boldsymbol{R} \mapsto \boldsymbol{R}$ be a strictly convex ${ }^{3}$, differentiable function. The pointwise Bregman divergence from $t_{0}$ to $t$ is a function $B R:(\boldsymbol{R} \times \boldsymbol{R}) \mapsto[0, \infty)$

\footnotetext{
${ }^{3}$ A function $f$ is said to be strictly convex if $\forall x_{1} \neq x_{2} \in \boldsymbol{R}, \forall t \in(0,1)$ :

$$
f\left(t x_{1}+(1-t) x_{2}\right)<t f\left(x_{1}\right)+(1-t) f\left(x_{2}\right) .
$$
}


defined for all $t_{0}, t \in \boldsymbol{R}$ :

$$
B R\left(t_{0} \| t\right)=f\left(t_{0}\right)-f(t)-f^{\prime}(t)\left(t_{0}-t\right)
$$

where $f^{\prime}$ is the first derivative of $f$. Figure 2.3 illustrates this relation.

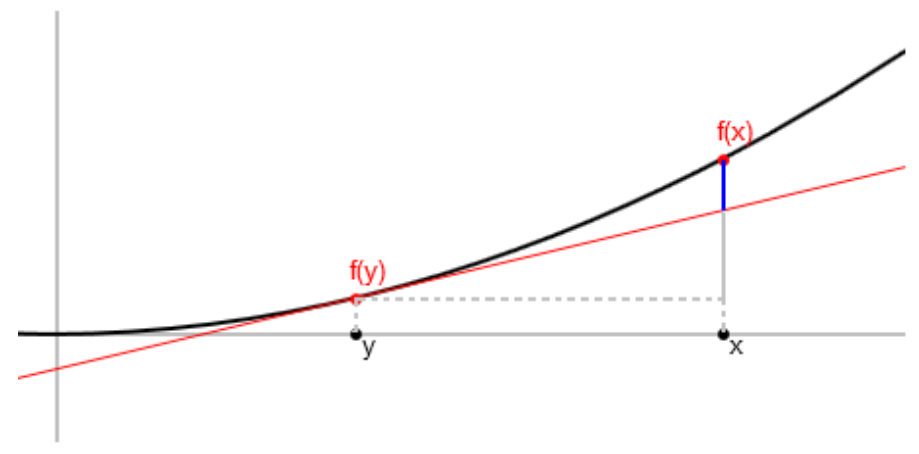

Figure 2.3: Bregman divergence $B R(x \| y)$ in blue. The value $f^{\prime}(y)(x-y)$ in solid grey.

Unlike distance metrics, the Bregman divergence $B R\left(t_{0} \| t\right)$ fails to be symmetric and does not obey the triangle inequality. The definition of $B R\left(t_{0} \| t\right)$ in Expression (2.15) is easily generalized to vectors in $\boldsymbol{R}^{d}$, in which case $f^{\prime}(t)\left(t_{0}-t\right)$ is replaced by $\nabla f(\boldsymbol{t})\left(\boldsymbol{t}_{0}-\boldsymbol{t}\right)$.

For our purposes, a more sophisticated Bregman divergence, defined on functions, is in order. Once again, let $f: \boldsymbol{R} \mapsto \boldsymbol{R}$ be a strictly convex, differentiable function. In addition, let $L: \mathcal{F} \mapsto \boldsymbol{R}$, be a functional defined on a convex set $\mathcal{F}$ of functions such that

$$
L(g)=\int f(g(x)) d \mu(x)
$$

where $\mu(x)$ is a $\sigma$-finite measure. Then, the "measure-theoretic" Bregman divergence (Stummer, 2007) from $g_{0}$ to $g$ is a functional $B R:(\mathcal{F} \times \mathcal{F}) \mapsto[0, \infty)$ defined for all $g_{0}, g \in \mathcal{F}$ :

$$
B R\left(g_{0} \| g\right)=L\left(g_{0}\right)-L(g)-\delta L\left(g, g_{0}-g\right)
$$

where $\delta L\left(g, g_{0}-g\right)$ is the directional derivative of $L$.

\subsubsection{Minimizing a Bregman Divergence from an Unknown Den- sity Ratio}

Here we show how to minimize an estimate of $B R\left(r_{0} \| r\right)$ over $r$, where $r_{0}(x)=\frac{p_{1}(x)}{p_{2}(x)} \in$ $\mathcal{F}$ is an unknown density ratio function and $r \in \mathcal{F}$. To begin with, Expression (2.16) can be expanded as

$$
B R\left(g_{0} \| g\right)=\int\left[f\left(g_{0}(x)\right)-f(g(x))-f^{\prime}(g(x))\left(g_{0}(x)-g(x)\right)\right] d \mu(x),
$$

where $f^{\prime}$ is the derivative of $f$. 
For $B R\left(r_{0} \| r\right)$, let us use the measure associated to the denominator density $p_{2}$, i.e $d \mu(x)=d F_{2}(x)$, where $F_{2}$ is the CDF associated to $p_{2}$. This way:

$$
B R\left(r_{0} \| r\right)=\int\left[f\left(r_{0}(x)\right)-f(r(x))-f^{\prime}(r(x))\left(r_{0}(x)-r(x)\right)\right] d F_{2}(x) .
$$

As we want to minimize $B R\left(r_{0} \| r\right)$ over $r$, the terms depending only on $r_{0}$ can be ignored. Rewriting $B R\left(r_{0} \| r\right)$ only with the terms that depend on $r$, we obtain

$$
B R^{\prime}\left(r_{0} \| r\right)=\int\left[f^{\prime}(r(x)) r(x)-f(r(x))\right] d F_{2}(x)-\int f^{\prime}(r(x)) r_{0}(x) d F_{2}(x) .
$$

Recall that $d F_{2}(x)=p_{2}(x) d x, p_{1}(x) d x=d F_{1}(x)$ and $r_{0}(x)=p_{1}(x) / p_{2}(x)$. Using the left-hand side of these equalities in place of their right-hand side, we arrive at

$$
B R^{\prime}\left(r_{0} \| r\right)=\int\left[f^{\prime}(r(x)) r(x)-f(r(x))\right] d F_{2}(x)-\int f^{\prime}(r(x)) d F_{1}(x) .
$$

Note that $r_{0}$ is absent from this last expression for $B R\left(r_{0} \| r\right)$. Using the law of large numbers, it is now possible to obtain an empirical estimator $\widehat{B R^{\prime}}\left(r_{0} \| r\right)$ for $B R^{\prime}\left(r_{0} \| r\right)$ based on samples $x_{1}, \ldots, x_{\ell} \stackrel{i . i . d}{\sim} F_{1}(x)$ and $x_{1}^{\prime}, \ldots, x_{n}^{\prime} \stackrel{\text { i.i.d }}{\sim} F_{2}(x)$ :

$$
\widehat{B R^{\prime}}\left(r_{0} \| r\right)=\frac{1}{n} \sum_{i=1}^{n}\left[f^{\prime}\left(r\left(x_{i}^{\prime}\right)\right) r\left(x_{i}^{\prime}\right)-f\left(r\left(x_{i}^{\prime}\right)\right)\right]-\frac{1}{\ell} \sum_{i=1}^{\ell} f^{\prime}\left(r\left(x_{i}\right)\right) .
$$

The question of whether $\ell, n \rightarrow \infty$ implies

$$
\left[\boldsymbol{r}_{\ell, n}=\underset{\boldsymbol{r}}{\arg \min } \widehat{B R^{\prime}}\left(r_{0} \| r\right)\right] \rightarrow r_{0}
$$

in some mode of convergence is not a simple one. We return to this question in Section 2.5.

\subsubsection{Previous Methods of Density Ratio Estimation}

Particular choices of $f$ in Expression (2.18) give rise to different categories of density ratio estimation methods. Here we present the ones appearing in our experimental evaluation in Chapter 3. For other instantiations, the reader is referred to (Sugiyama et al., 2012).

Let us start with the choice $f(t)=\frac{1}{2}(t-1)^{2}$. Plugging it into Expression (2.18):

$$
\widehat{B R^{\prime}}\left(r_{0} \| r\right)=\frac{1}{2 n} \sum_{i=1}^{n} r\left(x_{i}^{\prime}\right)^{2}-\frac{1}{\ell} \sum_{i=1}^{\ell} r\left(x_{i}\right)
$$

Minimization of Expression (2.20) over $r$ is the starting point for a category of methods 
termed Least-Squares Importance Filtering (LSIF) (Kanamori et al., 2009). What differentiates the methods within this category is the particular regularization employed. This minimization is also conducted implicitly in the Kernel Mean Matching method (Gretton et al., 2009).

On a side note, if we plug $f(t)=\frac{1}{2}(t-1)^{2}$ directly into Expression (2.17), what we obtain is the squared error weighted by the denominator density:

$$
B R\left(r_{0} \| r\right)=\int\left(r_{0}(x)-r(x)\right)^{2} p_{2}(x) d x
$$

That is, by minimizing Expression (2.20) over $r$ we are minimizing Expression (2.21) empirically.

Now, let us consider $f(t)=t \log t-t$ and plug it into Expression (2.18):

$$
\widehat{B R^{\prime}}\left(r_{0} \| r\right)=\frac{1}{n} \sum_{i=1}^{n} r\left(x_{i}^{\prime}\right)-\frac{1}{\ell} \sum_{i=1}^{\ell} \log r\left(x_{i}\right) .
$$

Minimization of Expression (2.22) is present in the category of methods based on the minimization of the Kullback-Leibler (KL) divergence from $r_{0}$ to $r$ (Sugiyama et al., 2008; Nguyen et al., 2008, 2010). For instance, by considering only positive solutions $r(x)>0$ - and the constraint

$$
\frac{1}{n} \sum_{i=1}^{n} r\left(x_{i}^{\prime}\right)=1
$$

one obtains from Expression (2.22) the Kullback-Leibler Importance Estimation Procedure (KLIEP) (Sugiyama et al., 2008):

$$
\begin{aligned}
\boldsymbol{r}_{\ell, n}=\underset{\boldsymbol{r}}{\arg \max } & \frac{1}{\ell} \sum_{i=1}^{\ell} \log r\left(x_{i}\right) \\
\text { s.t. } & \frac{1}{n} \sum_{i=1}^{n} r\left(x_{i}^{\prime}\right)=1 \\
& r\left(x_{i}^{\prime}\right) \geq 0, \quad i=1, \ldots, n .
\end{aligned}
$$

\subsection{Final Remarks}

The problem of density ratio estimation has received a lot of attention lately. The approach presented in Section 2.4 and the methods therein were all developed in the late 2000's. It is worth noting, however, that the first rigorous treatment of the subject is that presented in (Vapnik, 1998, Chapter 7), which extends preliminary results by Stefanyuk (1986).

Other related work from the past century include (Villalobos and Wahba, 1987) and 
(Silverman, 1978), both focusing on the direct estimation of the logarithm of a density ratio. Their methods are similar to the ones presented in Section 2.4.

Going back to the question posed at the end of Section 2.4.2, we note that there are no general results establishing whether or not Expression (2.19) holds. In other words, there may be assignments of valid functions $f$ in Expression (2.18) that do not yield the desired convergence to the density ratio $r_{0}$. There are some specific results establishing the convergence in probability of $r_{\ell, n}$ to the density ratio $r_{0}$ provided some assumptions hold. Kanamori et al. (2009) and Nguyen et al. (2008) provide such results for LSIF- and KL-methods. For LSIF methods, however, convergence is guaranteed to take place only in the metric induced by the $L^{2}\left(F_{2}\right)$ norm, that is, the density ratio function should have a norm in the $L^{2}\left(F_{2}\right)$ space. For KL methods, in turn, one of the assumptions is the ratio function to be bounded below and above. In both cases, there are density ratio functions that cannot be estimated by these methods as they violate these assumptions.

To the best of our knowledge, the stochastic method presented in Section 2.3 is the only approach to density ratio estimation that neither imposes a particular distance metric nor restricts the values of the density ratio function. The methods based on minimization of the Bregman divergence, however, have no such general applicability. As such, the stochastic method has a wider applicability in density ratio estimation.

In this chapter we focused on the theoretical properties of density ratio methods. In the next chapter, we compare their computational properties as well as their experimental performance in density ratio estimation. 


\section{Chapter 3}

\section{Algorithms for Stochastic Density Ratio Estimation}

In this chapter, our goal is to develop computational methods for estimating the density ratio function $r(x)=\frac{p_{1}(x)}{p_{2}(x)}$ using the stochastic theory of density ratio estimation - Chapter 2. According to this theory, the density ratio estimation problem is that of solving the integral equation

$$
\int_{-\infty}^{x} r(t) d F_{2}(t)=F_{1}(x)
$$

where $F_{1}$ and $F_{2}$ are the cumulative distribution functions - CDFs - associated with $p_{1}$ and $p_{2}$, respectively. As $F_{1}$ and $F_{2}$ are unknown, sampled data $x_{1}, \ldots, x_{\ell} \stackrel{\text { i.i.d }}{\sim} F_{1}(x)$ and $x_{1}^{\prime}, \ldots, x_{n}^{\prime} \stackrel{i . i . d}{\sim} F_{2}(x)$ are used to construct the empirical cumulative distribution functions - ECDFs $-F_{1, \ell}$ and $F_{2, n}$, which approximate the real CDFs $F_{1}$ and $F_{2}$. The ECDFs are then used to construct approximations to the left- and right-hand side of the integral equation $A r=F_{1}$ in Expression (3.1):

$$
\begin{gathered}
\left(A_{n} r\right)(x)=\int_{-\infty}^{x} r(t) d F_{2, n}(t)=\frac{1}{n} \sum_{i=1}^{n} r\left(x_{i}^{\prime}\right) \theta\left(x-x_{i}^{\prime}\right), \\
F_{1, \ell}(x)=\frac{1}{\ell} \sum_{i=1}^{\ell} \theta\left(x-x_{i}\right),
\end{gathered}
$$

where $\theta(u)=\left\{\begin{array}{ll}1, & \text { if } u \geq 0 \\ 0, & \text { otherwise }\end{array}\right.$.

Solving the integral equation in Expression (3.1) via its approximation $A_{n} r=F_{1, \ell}$ is an ill-posed problem whenever we consider a wide set $\mathcal{S}_{1}^{*}$ of candidate solutions. As argued in Section 2.3, if the solution belongs to $\mathcal{S}_{1}^{*}$, the regularization method can be employed for retrieving it from $\mathcal{S}_{1}^{*}$. For $\ell, n \rightarrow \infty$, this method asks for the solution of 
the minimization problem

$$
r_{\ell, n}=\underset{r \in \mathcal{S}_{1}^{*}}{\arg \min }\left[\rho_{2}\left(A_{n} r, F_{1, \ell}\right)^{2}+\gamma_{\ell, n} \Omega(r)\right] .
$$

Expression (3.2) relates the elements of the stochastic density ratio estimation approach. In order to develop a computational method on its grounds, we need to properly choose three entities:

1. a distance metric $\rho_{2}$;

2. a regularizing functional $\Omega$;

3. a regularization parameter $\gamma_{\ell, n}$.

Different instantiations of these entities yield different computational methods of density ratio estimation.

This chapter is organized as follows. Sections 3.1, 3.2, and 3.4 are devoted to the instantiation of entities 1, 2, and 3, respectively. Section 3.3 covers the optimization problems arising from the instantiation of $\rho_{2}$ and $\Omega$, contrasting these optimization problems with those arising from previous methods of density ratio estimation. Section 3.5 reports some experiments conducted on synthetic data to compare the performance of the proposed methods against each other and also against previous methods.

\subsection{Distance between ECDF Functions}

The role of $\rho_{2}\left(A_{n} r, F_{1, \ell}\right)$ in Expression (3.2) is to quantitatively assess how far apart are the empirical functions $A_{n} r$ and $F_{1, \ell}$. In this section, we deal with distance metrics induced by a norm. This way, let us rewrite the term $\rho_{2}\left(A_{n} r, F_{1, \ell}\right)^{2}$ in Expression (3.2):

$$
\rho_{2}\left(A_{n} r, F_{1, \ell}\right)^{2}=\left\|\frac{1}{n} \sum_{i=1}^{n} r\left(x_{i}^{\prime}\right) \theta\left(x-x_{i}^{\prime}\right)-\frac{1}{\ell} \sum_{i=1}^{\ell} \theta\left(x-x_{i}\right)\right\|^{2} .
$$

In what follows, Expression (3.3) is written in closed-form thanks to the chosen norm in the space of functions $\mathcal{S}_{2}$.

\subsubsection{V-Matrices}

Let us start by assuming that all functions $f \in S_{2}$ are such that $f:[0,1]^{d} \mapsto \boldsymbol{R}$. We shall extend the domain of $f$ after working out this simpler case. 
Using the $L^{2}$ norm in the space of functions $S_{2}$, we obtain:

$$
\rho_{2}\left(A_{n} r, F_{1, \ell}\right)^{2}=\int_{0}^{1}\left[\frac{1}{n} \sum_{i=1}^{n} r\left(x_{i}^{\prime}\right) \theta\left(x-x_{i}^{\prime}\right)-\frac{1}{\ell} \sum_{i=1}^{\ell} \theta\left(x-x_{i}\right)\right]^{2} d x
$$

Expression (3.4) can be written as

$$
\rho_{2}\left(A_{n} r, F_{1, \ell}\right)^{2}=\int_{0}^{1} a^{2} d x-2 \int_{0}^{1} a b d x+\int_{0}^{1} b^{2} d x
$$

where

$$
\begin{array}{ll}
a=\frac{1}{n} \sum_{i=1}^{n} r\left(x_{i}^{\prime}\right) \theta\left(x-x_{i}^{\prime}\right), & x_{1}^{\prime}, \ldots, x_{n}^{\prime} \stackrel{\text { i.i.d }}{\sim} F_{2}(x), \\
b=\frac{1}{\ell} \sum_{i=1}^{\ell} \theta\left(x-x_{i}\right), & x_{1}, \ldots, x_{\ell} \stackrel{\text { i.i.d }}{\sim} F_{1}(x) .
\end{array}
$$

The last term in Expression (3.5) does not depend on $r$ and thus can be ignored for minimization over $r$. In what follows, we use the notation $\left(t_{1} \vee t_{2}\right)=\max \left(t_{1}, t_{2}\right)$. The first two terms of Expression (3.5) are

$$
\begin{aligned}
& \int_{0}^{1} a^{2} d x= \\
& =\frac{1}{n^{2}} \sum_{i=1}^{n} \sum_{j=1}^{n} r\left(x_{i}^{\prime}\right) r\left(x_{j}^{\prime}\right) \int_{0}^{1} \theta\left(x-x_{i}^{\prime}\right) \theta\left(x-x_{j}^{\prime}\right) d x \\
& =\frac{1}{n^{2}} \sum_{i=1}^{n} \sum_{j=1}^{n} r\left(x_{i}^{\prime}\right) r\left(x_{j}^{\prime}\right) \int_{\left(x_{i}^{\prime}{ }_{i} \vee x_{j}^{\prime}\right)}^{1} \int_{\left(x_{i}^{\prime}{ }_{i} \vee x_{j}^{\prime}{ }_{j}\right)}^{1} d x^{1} \ldots d x^{d} \\
& =\frac{1}{n^{2}} \sum_{i=1}^{n} \sum_{j=1}^{n} r\left(x_{i}^{\prime}\right) r\left(x_{j}^{\prime}\right) \prod_{k=1}^{d}\left[1-\left(x_{i}^{\prime k} \vee x_{j}^{\prime k}\right)\right] \\
& -2 \int_{0}^{1} a b d x= \\
& =-\frac{2}{n \ell} \sum_{i=1}^{n} \sum_{j=1}^{\ell} r\left(x_{i}^{\prime}\right) \int_{0}^{1} \theta\left(x-x_{i}^{\prime}\right) \theta\left(x-x_{j}\right) d x \\
& =-\frac{2}{n \ell} \sum_{i=1}^{n} \sum_{j=1}^{\ell} r\left(x_{i}^{\prime}\right) \int_{\left(x_{i}^{\prime 1} \vee x_{j}^{1}\right)}^{1} \ldots \int_{\left(x_{i}^{\prime} \vee x_{j}^{d}\right)}^{1} d x^{1} \ldots d x^{d} \\
& =-\frac{2}{n \ell} \sum_{i=1}^{n} \sum_{j=1}^{n} r\left(x_{i}^{\prime}\right) \prod_{k=1}^{d}\left[1-\left(x_{i}^{\prime k} \vee x_{j}^{k}\right)\right] .
\end{aligned}
$$


Let us denote by $v_{i j}^{\prime \prime}$ the values

$$
v_{i j}^{\prime \prime}=\prod_{k=1}^{d}\left[1-\left(x_{i}^{\prime k} \vee x_{j}^{\prime k}\right)\right], \quad \quad x_{i}^{\prime}, x_{j}^{\prime} \sim F_{2}(x)
$$

and by $V^{\prime \prime}$ the $n \times n$-dimensional matrix of elements $v_{i j}^{\prime \prime}$. Also, let us denote by $v_{i j}^{\prime}$ the values

$$
v_{i j}^{\prime}=\prod_{k=1}^{d}\left[1-\left(x_{i}^{\prime k} \vee x_{j}^{k}\right)\right], \quad \quad x_{i}^{\prime} \sim F_{2}(x), x_{j} \sim F_{1}(x)
$$

and by $V^{\prime}$ the $n \times \ell$-dimensional matrix of elements $v_{i j}^{\prime}$.

After these derivations, Expression (3.4) has the following closed form:

$$
\rho_{2}\left(A_{n} r, F_{1, \ell}\right)^{2}=\frac{1}{2} \sum_{i=1}^{n} \sum_{j=1}^{n} r\left(x_{i}^{\prime}\right) r\left(x_{j}^{\prime}\right) v_{i j}^{\prime \prime}-\frac{n}{\ell} \sum_{i=1}^{n} r\left(x_{i}^{\prime}\right) \sum_{j=1}^{n} v_{i j}^{\prime}+\text { const. }
$$

Alternatively, using vector-matrix notation:

$$
\rho_{2}\left(A_{n} r, F_{1, \ell}\right)^{2}=\frac{1}{2} \boldsymbol{r}^{\top} V^{\prime \prime} \boldsymbol{r}-\frac{n}{\ell} \boldsymbol{r}^{\top} V^{\prime} \mathbf{1}_{\ell}+\text { const },
$$

where $\boldsymbol{r}$ denotes the $n$-dimensional vector $\left[r\left(x_{1}^{\prime}\right), \ldots, r\left(x_{n}^{\prime}\right)\right]^{\top}$ and $\mathbf{1}_{\ell}$ denotes the $\ell$-dimensional vector $[1, \ldots, 1]^{\top}$.

The matrices $\mathbf{V}^{\prime \prime}$ and $\mathbf{V}^{\prime}$ so obtained capture the geometry of the observed data in relation to the chosen norm in space $\mathcal{S}_{2}$ and the integral equation in Expression (3.1). By choosing another $L^{2}$-type norm, we end up with different elements $v_{i j}^{\prime \prime}$ and $v_{i j}^{\prime}$, while retaining the vector-matrix notation in Expression (3.7).

For instance, let us consider the standard $L^{2}$ norm with the data in a box $\left[s^{1}, u^{1}\right] \times$ $\ldots \times\left[s^{d}, u^{d}\right], s^{k}, u^{k} \in \boldsymbol{R}$, which is the general case of a distribution on a bounded support. Then,

$$
v_{i j}^{\prime \prime}=\prod_{k=1}^{d}\left[u^{k}-\left(x_{i}^{\prime k} \vee x_{j}^{\prime k}\right)\right] \text { and } v_{i j}^{\prime}=\prod_{k=1}^{d}\left[u^{k}-\left(x_{i}^{\prime k} \vee x_{j}^{k}\right)\right]
$$

Now, for the $L^{2}\left(F_{2, n}\right)$ norm, we have

$$
\begin{aligned}
\rho_{2}\left(A_{n} r, F_{1, \ell}\right)^{2} & =\int_{-\infty}^{\infty}\left[\frac{1}{n} \sum_{i=1}^{n} r\left(x_{i}^{\prime}\right) \theta\left(x-x_{i}^{\prime}\right)-\frac{1}{\ell} \sum_{i=1}^{\ell} \theta\left(x-x_{i}\right)\right]^{2} d F_{2, n}(x) \\
& =\frac{1}{n} \sum_{m=1}^{n}\left[\frac{1}{n} \sum_{i=1}^{n} r\left(x_{i}^{\prime}\right) \theta\left(x_{m}^{\prime}-x_{i}^{\prime}\right)-\frac{1}{\ell} \sum_{i=1}^{\ell} \theta\left(x_{m}^{\prime}-x_{i}\right)\right]^{2} .
\end{aligned}
$$


After some derivations, the $V$-matrices elements for the $L^{2}\left(F_{2, n}\right)$ norm are

$$
v_{i j}^{\prime \prime}=\frac{1}{n} \sum_{m=1}^{n} \prod_{k=1}^{d} \theta\left(x_{m}^{\prime k}-\left(x_{i}^{\prime k} \vee x_{j}^{\prime k}\right)\right) \text { and } v_{i j}^{\prime}=\frac{1}{n} \sum_{m=1}^{n} \prod_{k=1}^{d} \theta\left({x^{\prime}}_{m}^{k}-\left(x_{i}^{\prime k} \vee x_{j}^{k}\right)\right)
$$

Note that the data does not need to be in a pre-specified box for this choice of norm.

Figure 3.1 contrasts the distances arising from the standard $L^{2}$ norm and the $L^{2}\left(F_{2, n}\right)$ norm. While the distance based on the former averages the discrepancy of $A_{n} r$ and $F_{1, \ell}$ at each point of the box (infinite set), the distance based on the latter averages the same discrepancy only at the finite set $\left\{x_{1}^{\prime}, \ldots, x_{n}^{\prime} \stackrel{\text { i.i.d }}{\sim} F_{2}(x)\right\}$.

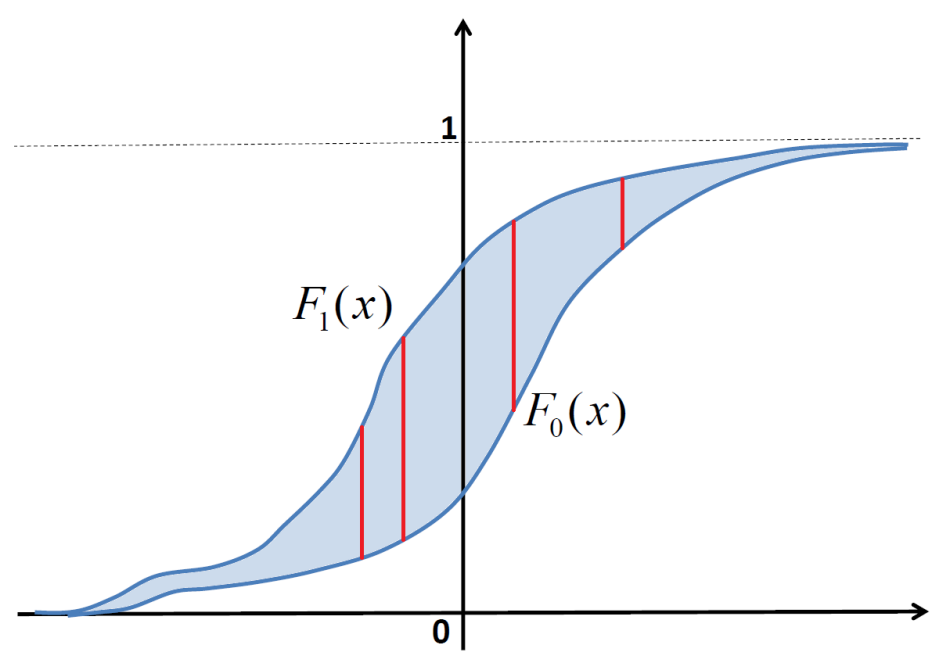

Figure 3.1: Distance between distribution functions. The standard $L^{2}$ norm averages the discrepancy of the two functions on the whole domain of their definition (shaded blue area). The $L^{2}\left(F_{2, n}\right)$ norm averages the discrepancy only at a finite number of points (red lines).

\subsubsection{More Advanced Distances}

Squared norms can always be employed for the stochastic density ratio estimation problem because the difference function $A_{n} r-F_{1, \ell}$ is always square integrable. In this setting, larger differences contribute more to the norm than smaller ones. In what follows, we show that this property of squared norms may cause distortions in density ratio estimation.

The previous section started out with a distance based on the standard $L^{2}$ norm Expression (3.4). This norm belongs to a general family of norms:

$$
\rho_{2}\left(A_{n} r, F_{1, \ell}\right)^{2}=\int_{0}^{1}\left[\frac{1}{n} \sum_{i=1}^{n} r\left(x_{i}^{\prime}\right) \theta\left(x-x_{i}^{\prime}\right)-\frac{1}{\ell} \sum_{i=1}^{\ell} \theta\left(x-x_{i}\right)\right]^{2} \mu(x) d x
$$

where $\mu(x)$ is any integrable weighting function. 
For $\mu(x)=1$, we retrieve the standard $L^{2}$ norm in Expression (3.4). For $\mu(x)=p_{2}(x)$, we end up with a norm for which its empirical approximation is the $L^{2}\left(F_{2, n}\right)$ norm in Expression (3.8). The goal is to choose a function $\mu(x)$ that better suites the density ratio estimation task.

Let us suppose that $F$ is a CDF and $F_{\ell}$ is its ECDF. Then, for any fixed point $x, \ell F_{\ell}(x)$ follows a Binomial distribution. Consequently, the variance of $F_{\ell}(x)$ can be defined by a value proportional to

$$
\epsilon(x)=F(x)[1-F(x)] .
$$

Let us use the distance metric in Expression (3.9) with the function $\mu(x)$ defined as:

$$
\mu(x)=\epsilon(x)^{-1}=\frac{1}{F_{1}(x)\left[1-F_{1}(x)\right]} .
$$

Thus, at every point $x$, the square of the difference between the two functions is measured relatively to the variance at this point. Such definition of distance metric reflects a uniform relative deviation between two functions. This (normalized) distance prevents relatively large deviations at the endpoints of the interval $[0,1]$.

In real scenarios, we do not know the cumulative distribution function $F_{1}(x)$. Therefore, in Expression (3.10), we use its empirical estimate $F_{1, \ell}(x)$. Let us construct the $V$-matrices elements for the distance in Expression (3.9) with $\mu(x)$ defined as in Expression (3.10). For simplicity, we provide them only for the one-dimensional case.

For the one-dimensional case, without loss of generality, let the observations sampled according to $F_{2}$ be ordered with respect to their values

$$
0 \leq x_{1}^{\prime} \leq x_{2}^{\prime} \leq \ldots \leq x_{n}^{\prime} \leq 1
$$

Consider the corresponding values $\mu\left(x_{m}^{\prime}\right), m=1, \ldots, n$ defined in Expression (3.10).

For any two adjacent elements $x_{m-1}^{\prime}$ and $x_{m}^{\prime}$ of the sequence (3.11) and any number $a \in[0,1]$, we define the value $\left(x_{m}^{\prime}-x_{m-1}^{\prime}\right)_{a}$ as

$$
\left(x_{m}^{\prime}-x_{m-1}^{\prime}\right)_{a}=\left\{\begin{array}{ll}
x_{m}^{\prime}-x_{m-1}^{\prime} & \text { if } x_{m-1}^{\prime}>a \\
x_{m}^{\prime}-a & \text { if } x_{m-1}^{\prime} \leq a
\end{array} .\right.
$$

Repeating the steps of the previous section, but now for the new distance formed by Expressions (3.9) and (3.10), we obtain

$$
\begin{aligned}
& v_{i j}^{\prime \prime}=\sum_{m: x_{m}^{\prime}>\left(x_{i}^{\prime} \vee x_{j}^{\prime}\right)} \mu\left(x_{m}^{\prime}\right)\left(x_{m}^{\prime}-x_{m-1}^{\prime}\right)_{\left(x_{i}^{\prime} \vee x_{j}^{\prime}\right)}, \\
& v_{i, j}^{\prime}=\sum_{m: x_{m}^{\prime}>\left(x_{i}^{\prime} \vee x_{j}\right)} \mu\left(x_{m}^{\prime}\right)\left(x_{m}^{\prime}-x_{m-1}^{\prime}\right)_{\left(x_{i}^{\prime} \vee x_{j}\right)} .
\end{aligned}
$$


Computing $v_{i, j}^{\prime \prime}$ and $v_{i, j}^{\prime}$ is unfeasible for high dimensional cases when $\mu(x)$ is set as in Expression (3.10). In these cases, it may be preferable to use

$$
\mu(x)=\frac{p_{2}(x)}{\epsilon(x)}=\frac{p_{2}(x)}{F_{1}(x)\left[1-F_{1}(x)\right]} .
$$

Plugging Expression (3.12) into Expression (3.9) and replacing the CDFs $F_{1}$ and $F_{2}$ by their respective ECDFs $F_{1, \ell}$ and $F_{2, n}$, we arrive at the following expressions for the $V$ matrices elements:

$v_{i j}^{\prime \prime}=\frac{1}{n} \sum_{m=1}^{n} \frac{1}{\epsilon\left(x_{m}^{\prime k}\right)} \prod_{k=1}^{d} \theta\left(x_{m}^{\prime k}-\left(x_{i}^{\prime k} \vee x_{j}^{\prime k}\right)\right)$ and $v_{i j}^{\prime}=\frac{1}{n} \sum_{m=1}^{n} \frac{1}{\epsilon\left(x_{m}^{\prime k}\right)} \prod_{k=1}^{d} \theta\left(x_{m}^{\prime k}-\left(x_{i}^{\prime k} \vee x_{j}^{k}\right)\right)$.

\subsection{Regularizing Functional}

We define the regularizing functional $\Omega(r)$ to be the square of the norm in a Hilbert space:

$$
\Omega(r)=\|r\|_{\mathcal{H}}^{2}=\langle r, r\rangle_{\mathcal{H}}
$$

We consider two different Hilbert spaces, which correspond to two different norms and types of prior knowledge about the solution ${ }^{1}$.

Let us first define $\Omega(r)$ in Expression (3.13) using the $L^{2}\left(F_{2, n}\right)$ norm, which is defined on a finite dimensional Hilbert space:

$$
\Omega(r)=\int r(t)^{2} d F_{2, n}(t)=\frac{1}{n} \sum_{i=1}^{n} r\left(x_{i}^{\prime}\right)^{2}=\frac{1}{n} \boldsymbol{r}^{\top} \boldsymbol{r} .
$$

This regularizer penalizes large values of the ratio function.

We also define $\Omega(r)$ in Expression (3.13) as a norm in a Reproducing Kernel Hilbert Space (RKHS), which is described next. Such a regularizer forces the ratio function to be at least continuous.

\subsubsection{Reproducing Kernel Hilbert Space and Its Norm}

An RKHS is defined by a positive definite kernel function $k(x, y)$ and an inner product $\langle,\rangle_{\mathcal{H}}$ for which the following reproducing property holds true:

$$
\langle f(x), k(x, y)\rangle_{\mathcal{H}}=f(y), \forall f \in \mathcal{H}
$$

Note that any positive definite function $k(x, y)$ has an expansion in terms of its eigen-

\footnotetext{
${ }^{1}$ Recall that the solution $r_{0}$ must have a norm in the corresponding space: $\left\langle r_{0}, r_{0}\right\rangle_{\mathcal{H}}=c_{0}<\infty$
} 
values $\lambda_{i} \geq 0$ and eigenfunctions $\phi_{i}(x)$ :

$$
k(x, y)=\sum_{i=1}^{\infty} \lambda_{i} \phi_{i}(x) \phi_{i}(y)
$$

Let us consider the set of functions

$$
f(x, c)=\sum_{i=1}^{\infty} c_{i} \phi_{i}(x)
$$

and the inner product

$$
\left\langle f\left(x, c^{*}\right), f\left(x, c^{* *}\right)\right\rangle_{\mathcal{H}}=\sum_{i=1}^{\infty} \frac{c_{i}^{*} c_{i}^{* *}}{\lambda_{i}} .
$$

Then, the kernel in Expression (3.16), the set of functions in Expression (3.17), and the inner product in Expression (3.18) define an RKHS. Indeed,

$$
\begin{aligned}
\langle f(x), k(x, y)\rangle_{\mathcal{H}} & =\left\langle\sum_{i=1}^{\infty} c_{i} \phi_{i}(x), \sum_{i=1}^{\infty} \lambda_{i} \phi_{i}(x) \phi_{i}(y)\right\rangle_{\mathcal{H}} \\
& =\sum_{i=1}^{\infty} \frac{c_{i} \lambda_{i} \phi_{i}(y)}{\lambda_{i}}=f(y) .
\end{aligned}
$$

Under mild conditions over the regularizer and restricting the space of candidate solutions to an RKHS, the solution of Expression (3.2) has the following form according to the representer theorem (Kimeldorf and Wahba, 1970):

$$
r(x)=\sum_{i=1}^{n} \alpha_{i} k\left(x_{i}^{\prime}, x\right), \quad x_{1}^{\prime}, \ldots, x_{n}^{\prime} \sim F_{2}(x) .
$$

Therefore, using Expressions (3.15) and (3.19), the norm in an RKHS has the form

$$
\langle r(x), r(x)\rangle_{\mathcal{H}}=\sum_{i, j=1}^{n} \alpha_{i} \alpha_{j} k\left(x_{i}^{\prime}, x_{j}^{\prime}\right)=\boldsymbol{\alpha}^{\top} K \boldsymbol{\alpha}
$$

where $\mathbf{K}$ denotes the $n \times n$-dimensional matrix with elements $k_{i j}=k\left(x_{i}^{\prime}, x_{j}^{\prime}\right)$ and $\boldsymbol{\alpha}$ denotes the $n$-dimensional vector $\left[\alpha_{1}, \ldots, \alpha_{n}\right]^{\top}$. We also choose this norm as a regularization functional, i.e $\Omega(r)=\boldsymbol{\alpha}^{\top} K \boldsymbol{\alpha}$.

\subsubsection{More Advanced Regularizers}

A possible variation on the regularizer in Expression (3.14) is to consider

$$
\Omega(r)=\int(r(t)-1)^{2} d F_{2, n}(t)=\frac{1}{n} \sum_{i=1}^{n}\left(r\left(x_{i}^{\prime}\right)-1\right)^{2}=\frac{1}{n}\left(\boldsymbol{r}^{\top} \boldsymbol{r}-\boldsymbol{r}^{\top} \mathbf{1}\right)+\text { const. }
$$


This regularizer penalizes density ratio values far from 1, whereas the one in Expression (3.14) penalizes density ratio values far from 0 . Choosing one of them is a matter of prior knowledge about the solution: whether the two densities are only slightly different or not. The regularizer in Expression (3.21) is more appropriate to the former case.

Another possibility is to not only penalize the magnitude of the density ratio values, as in Expression (3.14), but also the smoothness of the density ratio function. While the regularizers in an RKHS may privilege smooth solutions, the norm in an RKHS does not control smoothness directly. The following regularizer is an attempt to control smoothness directly:

$$
\begin{aligned}
\Omega(r) & =\int[\nabla r(x)]^{2} d F_{2, n}(x) \\
& \approx \sum_{i=1}^{n} \sum_{\substack{j=1 \\
j \neq i}}^{n}\left(\frac{r\left(x_{i}^{\prime}\right)-r\left(x_{j}^{\prime}\right)}{\left\|x_{i}-x_{j}\right\|}\right)^{2} \\
& =2\left(\sum_{i=1}^{n} \sum_{\substack{j=1 \\
j \neq i}}^{n} \frac{r\left(x_{i}^{\prime}\right) r\left(x_{i}^{\prime}\right)}{\left\|x_{i}-x_{j}\right\|^{2}}-\sum_{i=1}^{n} \sum_{\substack{j=1 \\
j \neq i}}^{n} \frac{r\left(x_{i}^{\prime}\right) r\left(x_{j}^{\prime}\right)}{\left\|x_{i}-x_{j}\right\|^{2}}\right) .
\end{aligned}
$$

Alternatively, using vector-matrix notation, $\Omega(r)=\boldsymbol{r}^{\top} G \boldsymbol{r}$, where the elements $g_{i j}$ of the $n \times n$-dimensional matrix $G$ are:

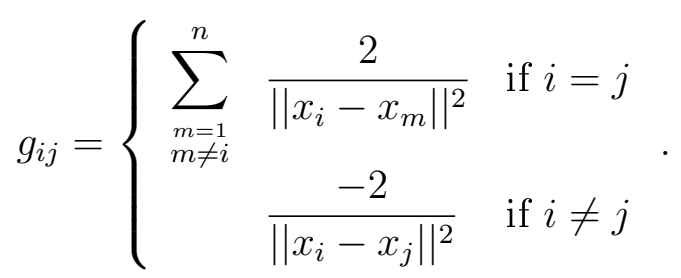

\subsection{Solving the Minimization Problem}

In this section, we obtain solutions of the minimization problem in Expression (3.2) for particular instances of $\rho_{2}$ and $\Omega$. Here, we suppose that an appropriate $\gamma_{\ell, n}$ has already been provided.

\subsubsection{Solution at Given Points (DRE-V)}

We rewrite the minimization problem in Expression (3.2) in an explicit form using Expressions (3.7) and (3.14). This leads to the following optimization problem:

$$
\mathcal{L}=\min _{\boldsymbol{r}}\left[\frac{1}{2} \boldsymbol{r}^{\top} V^{\prime \prime} \boldsymbol{r}-\frac{n}{\ell} \boldsymbol{r}^{\top} V^{\prime} \mathbf{1}_{\ell}+\frac{\gamma}{n} \boldsymbol{r}^{\top} \boldsymbol{r}\right]
$$


The minimum of this functional has the form

$$
\boldsymbol{r}=\frac{n}{\ell}\left(V^{\prime \prime}+\frac{2 \gamma}{n} I\right)^{-1} V^{\prime} \mathbf{1}_{\ell}
$$

which can be computed by solving the corresponding system of linear equations $-O\left(n^{3}\right)$ complexity.

In order to obtain a more accurate solution, we can take into account our prior knowledge that

$$
\begin{array}{r}
r\left(x_{i}^{\prime}\right) \geq 0, \quad i=1, \ldots, n, \\
\frac{1}{n} \sum_{i=1}^{n} r\left(x_{i}^{\prime}\right)=1 .
\end{array}
$$

In this case, any standard quadratic programming package can be used to find the solution (vector $\left.\left[r\left(x_{1}^{\prime}\right), \ldots, r\left(x_{n}^{\prime}\right)\right]\right)$.

The optimization problem in Expression (3.23), namely DRE-V, has a structure similar to that of the Kernel Mean Matching - KMM - method (Gretton et al., 2009). The major differences are:

- DRE-V completely specifies the kernel function to be used, which is the function that computes the elements of the $V$-matrices - Section 3.1;

- DRE-V includes regularization, which is absent from KMM.

\subsubsection{Solution in RKHS (DRE-VK)}

In order to ensure smoothness of the density ratio obtained from the optimization problem in Expression (3.2), we look for a function in an RKHS defined by a kernel. According to the representer theorem (Kimeldorf and Wahba, 1970), the function has the form

$$
r(x)=\sum_{i=1}^{n} \alpha_{i} k\left(x_{i}^{\prime}, x\right)
$$

We rewrite the minimization problem in Expression (3.2) in explicit form using Expressions (3.7) and (3.20). Since $\boldsymbol{r}=K \boldsymbol{\alpha}$, we obtain the following optimization problem:

$$
\mathcal{L}=\min _{\boldsymbol{\alpha} \in \mathbf{R}^{n}}\left[\frac{1}{2} \boldsymbol{\alpha}^{\top} K V^{\prime \prime} K \boldsymbol{\alpha}-\frac{n}{\ell} \boldsymbol{\alpha}^{\top} K V^{\prime} \mathbf{1}_{\ell}+\gamma \boldsymbol{\alpha}^{\top} K \boldsymbol{\alpha}\right] .
$$

The minimum of this functional has the form

$$
\boldsymbol{\alpha}=\frac{n}{\ell}\left(V^{\prime \prime} K+2 \gamma I\right)^{-1} V^{\prime} \mathbf{1}_{\ell}
$$


which can also be computed by solving the corresponding system of linear equations.

The optimization problem in Expression (3.27), namely DRE-VK, has a structure similar to the structure of the unconstrained Least-Squares Importance Filtering - uLSIF - method (Kanamori et al., 2009). Thus, the computational complexity of both methods are in an equal footing. The major differences between these methods are:

- uLSIF uses identity matrices $I_{n \times n}$ and $I_{n \times \ell}$ in lieu of $V^{\prime \prime}$ and $V^{\prime}$ of DRE-VK;

- DRE-VK uses the regularization functional $\boldsymbol{\alpha}^{\top} K \boldsymbol{\alpha}$, whereas uLSIF uses $\boldsymbol{\alpha}^{\top} \boldsymbol{\alpha}$.

In order to use our prior knowledge about the non-negativity of the solution, one can restrict the set of functions in Expression (3.26) with conditions

$$
\alpha_{i} \geq 0 \text { and } k\left(x_{i}^{\prime}, x\right) \geq 0, \quad i=1, \ldots, n
$$

We mention en passant that the algorithms used to solve the optimization problem arising from the Kullback-Leibler Importance Estimation procedure - KLIEP (Sugiyama et al., 2008) are more computationally demanding than the ones required to solve the optimization problems described in this section, which are instances of a quadratic optimization task.

\subsection{Selection of the Regularization Constant}

According to the theoretical results presented in Section 2.3, the regularization constant $\gamma$ should satisfy the following conditions, where $m=\min (\ell, n)$ :

$$
\gamma_{\ell, n} \stackrel{m \rightarrow \infty}{\longrightarrow} 0 \text { and } m \gamma_{\ell, n} \stackrel{m \rightarrow \infty}{\longrightarrow} \infty
$$

In what follows, we describe how we choose $\gamma$ for finite $n$ and $\ell$ in our experiments. For comparison purposes, we employ the same technique for parameter selection as the uLSIF method.

\subsubsection{Cross-Validation for DRE-VK}

For the optimization problem in Expression (3.27), we choose the regularization constant $\gamma$ using $k$-fold cross-validation based on the minimization of the least-squares criterion (Kanamori et al., 2009):

$$
\begin{aligned}
J_{0} & =\frac{1}{2} \int\left[r(x)-r_{0}(x)\right]^{2} p_{2}(x) d x \\
& =\frac{1}{2} \int r(x)^{2} p_{2}(x) d x-\int r(x) p_{2}(x) d x+\text { const. }
\end{aligned}
$$


We partition the following data sets

$$
\begin{aligned}
& \mathcal{X}=\left\{x_{1}, \ldots, x_{\ell} \stackrel{i . i . d}{\sim} F_{1}(x)\right\} \quad \text { and } \\
& \mathcal{X}^{\prime}=\left\{x_{1}^{\prime}, \ldots, x_{n}^{\prime} \stackrel{i . i . d}{\sim} F_{2}(x)\right\}
\end{aligned}
$$

into $k$ disjoint sets

$$
\mathcal{Z}_{i}=\left\{z_{1, i}, \ldots, z_{\ell / k, i}\right\} \quad \text { and } \quad \mathcal{Z}_{i}^{\prime}=\left\{z_{1, i}^{\prime}, \ldots, z_{n / k, i}^{\prime}\right\}
$$

Using samples $\mathcal{X} \backslash \mathcal{Z}_{i}$ and $\mathcal{X}^{\prime} \backslash \mathcal{Z}_{i}^{\prime}$, a solution to the DRE-VK minimization problem is constructed for constant $\gamma$ :

$$
\boldsymbol{\alpha}_{\gamma}=\frac{n_{i}}{\ell_{i}}\left(V_{n_{i} \times n_{i}}^{\prime \prime} K_{i}+2 \gamma I\right)^{-1} V_{n_{i} \times \ell_{i}}^{\prime} \mathbf{1}_{\ell_{i}}
$$

After that, the solution is evaluated by the empirical least-squares criterion on samples $\mathcal{Z}_{i}$ and $\mathcal{Z}_{i}^{\prime}$ :

$$
J_{i}^{\gamma}=\frac{1}{2} \sum_{i=1}^{n / k} r_{\gamma}\left(z_{i, m}^{\prime}\right)^{2}-\frac{n}{\ell} \sum_{i=1}^{\ell / k} r_{\gamma}\left(z_{i, m}\right) .
$$

This procedure is conducted for each $m=1, \ldots, k$.

Then, from a set of regularization constant candidates $\Gamma=\left\{\gamma_{1}, \ldots, \gamma_{m}\right\}$, we select $\gamma^{*}$ that minimizes the least-squares criterion over all folds, i.e,

$$
\gamma^{*}=\underset{\gamma \in \Gamma}{\arg \min } \sum_{i=1}^{k} J_{i}^{\gamma} .
$$

We obtain the final solution using the selected $\gamma^{*}$ and unpartitioned samples $\mathcal{X}, \mathcal{X}^{\prime}$ :

$$
\boldsymbol{\alpha}=\frac{n}{\ell}\left(V^{\prime \prime} K+2 \gamma^{*} I\right)^{-1} V^{\prime} \mathbf{1}_{\ell}
$$

\subsubsection{Cross-Validation for DRE-V}

The aforementioned procedure for $\gamma$ selection is not readily available for estimation of values of the density ratio function at given points. However, we take advantage of the fact that finding a minimum of

$$
\mathcal{L}=\min _{\boldsymbol{\alpha} \in \mathbf{R}^{n}}\left[\frac{1}{2} \boldsymbol{\alpha}^{\top} V^{\prime \prime} V^{\prime \prime} \boldsymbol{\alpha}-\frac{n}{\ell} \boldsymbol{\alpha}^{\top} V^{\prime} \mathbf{1}+\frac{\gamma}{n} \boldsymbol{\alpha}^{\top} V^{\prime \prime} \boldsymbol{\alpha}\right]
$$

leads to the same solution at given points as the optimization problem in Expression (3.24) if the same value of $\gamma$ is used for both problems. 
Indeed, the minimum of the problem in Expression (3.31) is reached at

$$
\boldsymbol{\alpha}_{\gamma}=\frac{n}{\ell}\left(V^{\prime \prime} V^{\prime \prime}+2 \gamma V^{\prime \prime}\right)^{-1} V^{\prime} \mathbf{1}_{\ell}
$$

Consequently, the solution at given points is:

$$
\begin{aligned}
\boldsymbol{r} & =V^{\prime \prime} \boldsymbol{\alpha}= \\
& =\frac{n}{\ell} V^{\prime \prime}\left(V^{\prime \prime} V^{\prime \prime}+\frac{2 \gamma}{n} V^{\prime \prime}\right)^{-1} V^{\prime} \mathbf{1}_{\ell}= \\
& =\frac{n}{\ell}\left(V^{\prime \prime}+\frac{2 \gamma}{n} I\right)^{-1} V^{\prime} \mathbf{1}_{\ell} .
\end{aligned}
$$

In order to choose $\gamma$ for DRE-V, we use the least-squares cross-validation procedure of the last section, but using Expression (3.32) instead of Expressions (3.29) and (3.30).

\subsubsection{More Advanced $\gamma$ Selection}

So far, we have employed $k$-fold cross-validation as the training/test strategy for choosing the regularization constant $\gamma$. One advantage of DRE-V is the availability of the relatively inexpensive leave-one-out cross-validation described in Section 6.2. Note that the unconstrained solution in Expression 3.24 and the Regularized Least Squares (RLS) regression method share a similar structure.

Applying the reasoning presented in Section 6.2, we arrive at the following expression for calculating $r_{l o o, \gamma}\left(x_{1}^{\prime}\right), \ldots, r_{l o o, \gamma}\left(x_{n}^{\prime}\right)$ (denominator points):

$$
r_{l o o, \gamma}\left(x_{i}\right)=\frac{r_{\gamma}\left(x_{i}\right)}{1-h_{j j}}
$$

where $r_{\gamma}\left(x_{1}^{\prime}\right), \ldots, r_{\gamma}\left(x_{n}^{\prime}\right)$ are obtained using Expression (3.24) and all available points, and $h_{j j}$ is the corresponding diagonal element of $\mathrm{H}$ :

$$
H=\left(V^{\prime \prime}+\frac{2 \gamma}{n} I\right)^{-1} V^{\prime \prime}
$$

For calculating $r_{l o o, \gamma}\left(x_{1}\right), \ldots, r_{l o o, \gamma}\left(x_{\ell}\right)$ (numerator points), let us assume without loss of generality that we are always leaving the point $x_{\ell}$ out. To calculate $r_{l o o, \gamma}\left(x_{i}\right)$ for each $x_{i}$, we exchange indices. This way, we first calculate

$$
\boldsymbol{\alpha}^{\gamma, \ell}=\frac{n}{\ell}\left(V^{\prime \prime} V^{\prime \prime}+2 \gamma V^{\prime \prime}\right)^{-1} V^{\prime} \mathbf{1}_{(\ell-1)}
$$

and then

$$
r_{l o o, \gamma}\left(x_{\ell}\right)=\sum_{i=1}^{n} \alpha_{i}^{\gamma, \ell} k\left(x_{i}^{\prime}, x_{\ell}\right) .
$$


Finally, we calculate the same least squares criterion

$$
J^{\gamma}=\frac{1}{2} \sum_{i=1}^{n} r_{l o o, \gamma}\left(x_{i}^{\prime}\right)^{2}-\frac{n}{\ell} \sum_{i=1}^{\ell} r_{l o o, \gamma}\left(x_{i}\right)
$$

and select $\gamma$ that minimizes this criterion.

Taking advantage of the structural similarity between DRE-V and the RLS method, we could try to leverage any of the parameter selection approaches dealt with in Chapter 6. However, as the experimental evaluation in Chapter 6 demonstrates, these other approaches do not outperform the leave-one-out cross-validation procedure.

In a different vein, suppose we knew that the distance $\rho_{2}\left(A_{n} r, F_{1, \ell}\right)$ - Section 3.1 is concentrated around a value $\delta(n, \ell)$. Then, in principle, we would be able to select a regularization parameter $\gamma$ that provided a solution $r_{\gamma}$ such that $\rho_{2}\left(A_{n} r, F_{1, \ell}\right) \approx \delta(n, \ell)$. This approach is also behind the idea of the so-called Morozov regularization, where information about the admissible values of $\rho_{2}\left(A_{n} r, F_{1, \ell}\right)$ is used as a regularization parameter. The problem in this approach is that a lot of effort should be put to normalize the value of $\rho_{2}\left(A_{n} r, F_{1, \ell}\right)$ across different data distributions $F_{1}$ and $F_{2}$.

Besides using the variance of the distribution function $F_{1}$ - Section 3.1.2 - , we could try other normalization schemes, like defining the region where the data is located more accurately. This can lead to the situation shown in Figure 3.2, where the data occupy only a small part of the space inside the box, leaving most of the box empty. The goal is to develop a method that estimates the function inside the box $A^{*}$ rather than $A$ (where a large part of the space is not relevant). One can construct something akin to the box $A^{*}$ by applying the Principal Component Analysis (PCA) method on all of the available points.

\subsection{Experiments}

In this section, we report experimental results for the methods of density ratio estimation introduced in this work: DRE-V (solution at given points) and DRE-VK (smooth solutions in RKHS). For the latter, we instantiated two versions: DRE-VK-INK, which uses the parameterless INK-splines kernel described in (Izmailov et al., 2013); and DREVK-RBF, which uses the Gaussian RBF kernel. In all cases, the regularization constant $\gamma$ was chosen by 5 -fold cross-validation. For DRE-VK-RBF, the extra "smoothing" parameter $\sigma^{2}$ was cross-validated along with the regularization constant $\gamma$.

For comparison purposes, we also ran experiments for the Kernel Mean Matching KMM - procedure (Gretton et al., 2009), the Unconstrained Least-Squares Importance Filtering - uLSIF — algorithm (Kanamori et al., 2009), and the Kullback-Leibler Importance Estimation Procedure - KLIEP - (Sugiyama et al., 2008). For uLSIF and 


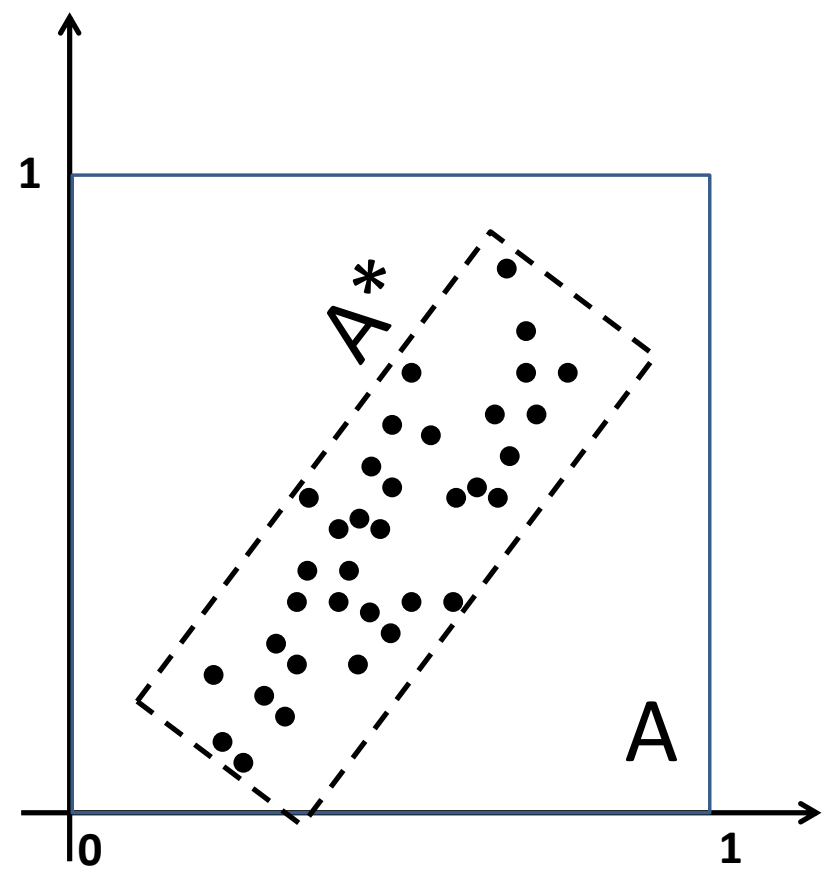

Figure 3.2: PCA transformation of a data set.

KLIEP, we used the code provided on the authors' website ${ }^{2}$, leaving parameters set to their default values with the exception of the number of folds in uLSIF, which was set ${ }^{3}$ to 5. For KMM, we followed the implementation used in the experimental section of (Gretton et al., 2009). KLIEP, uLSIF, and KMM traditionally use Gaussian RBF kernels. For KLIEP and uLSIF, selection of $\sigma^{2}$ was carried out by cross-validation, whereas in KMM $2 \sigma^{2}$ was set to the median distance between the input points.

We chose not to enforce the constraints in Expression (3.25) for DRE-V or DRE-VK-* in order to conduct a fair comparison with respect to uLSIF. We note that KMM and KLIEP do enforce

$$
r\left(x_{i}^{\prime}\right) \geq 0, \quad i=1, \ldots, n .
$$

The experiments were conducted on synthetic data described in Table 3.1. Models 4 and 6 were taken from the experimental evaluation of previously proposed methods (Kanamori et al., 2009). For diversity, we also considered heavy-tailed distributions, distributions on a bounded support, and a distribution which is not solvable in $L^{2}\left(F_{2}\right)$, hence, incompatible for estimation with DRE-V, KMM, uLSIF, and KLIEP.

For each model, we sampled $\ell$ points from $p_{1}(x)$ and another $n$ points from $p_{2}(x)$, with $\ell=n=m$ varying in $\{50,100,200\}$ for unidimensional data and $\{100,200,500\}$ for 20 -dimensional data. For each $m$, we performed 20 independent draws. A constant was added to each element of the sample in order to obtain values in the range $[0, u]$ in each coordinate, where $u$ was the largest value in the sample.

\footnotetext{
${ }^{2}$ http://sugiyama-www.cs.titech.ac.jp/ sugi/software/

${ }^{3}$ In our experiments, 5 -fold uLSIF performed better than the default leave-one-out uLSIF
} 
Table 3.1: Synthetic models

\begin{tabular}{|c|c|c|c|c|}
\hline Model \# & Dim. & $p_{1}(x)$ & $p_{2}(x)$ & Characteristics \\
\hline 1 & 1 & $\begin{array}{c}\text { Beta } \\
(0.5,0.5)\end{array}$ & $\begin{array}{l}\text { Uniform } \\
(0,1)\end{array}$ & Solution not in $L^{2}\left(F_{2}\right)$ \\
\hline 2 & 1 & $\begin{array}{l}\text { Beta } \\
(2,2)\end{array}$ & $\begin{array}{l}\text { Uniform } \\
(0,1)\end{array}$ & Bounded Support - BS \\
\hline 3 & 1 & $\begin{array}{l}\text { Beta } \\
(2,2)\end{array}$ & $\begin{array}{c}\text { Beta } \\
(0.5,0.5)\end{array}$ & $\mathrm{BS}$ \\
\hline 4 & 1 & $\begin{array}{c}\text { Gaussian } \\
(2,1 / 4)\end{array}$ & $\begin{array}{c}\text { Gaussian } \\
(1,1 / 2)\end{array}$ & $\begin{array}{c}\text { Unbounded Support - US } \\
\text { Light Tails - LT }\end{array}$ \\
\hline 5 & 1 & $\begin{array}{l}\text { Laplace } \\
(2,1 / 4)\end{array}$ & $\begin{array}{l}\text { Laplace } \\
(1,1 / 2)\end{array}$ & $\begin{array}{c}\text { US } \\
\text { Heavy Tails }- \text { HT }\end{array}$ \\
\hline 6 & 20 & $\begin{array}{c}\text { Gaussian } \\
\left([1,0, \ldots, 0], I_{20}\right)\end{array}$ & $\begin{array}{c}\text { Gaussian } \\
\left([0,0, \ldots, 0], I_{20}\right)\end{array}$ & $\begin{array}{l}\text { High Dimensional - HD } \\
\text { US, LT }\end{array}$ \\
\hline 7 & 20 & $\begin{array}{c}\text { Laplace } \\
\left([1,0, \ldots, 0], I_{20}\right)\end{array}$ & $\begin{array}{c}\text { Laplace } \\
\left([0,0, \ldots, 0], I_{20}\right)\end{array}$ & $\begin{array}{c}\mathrm{HD} \\
\mathrm{US}, \mathrm{HT}\end{array}$ \\
\hline
\end{tabular}

We evaluated the estimated density ratio at the points sampled from $p_{2}(x)$, since most applications require the estimation of the density ratio only at those points. Accordingly, we compared the estimate $\boldsymbol{r}=\left[r\left(x_{1}^{\prime}\right), \ldots, r\left(x_{m}^{\prime}\right)\right]^{\top}$ to the actual density ratio

$$
\boldsymbol{r}_{\mathbf{0}}=\left[\frac{p_{1}\left(x_{1}^{\prime}\right)}{p_{2}\left(x_{1}^{\prime}\right)}, \ldots, \frac{p_{1}\left(x_{m}^{\prime}\right)}{p_{2}\left(x_{m}^{\prime}\right)}\right]^{\top}
$$

using the normalized root mean squared error (NRMSE):

$$
N R M S E\left(\boldsymbol{r}, \boldsymbol{r}_{\mathbf{0}}\right)=\frac{\left\|\boldsymbol{r}-\boldsymbol{r}_{0}\right\|_{2}}{\left\|\boldsymbol{r}_{\mathbf{0}}\right\|_{2}}
$$

Experimental results are summarized in Table 3.2. For each $m$, we report the mean NRMSE and the standard deviation over the independent draws. The best result across the methods are highlighted in bold. Furthermore, the worst result obtained by the methods of density ratio estimation based on the $V$-matrix which is better than all of the corresponding results obtained by the previously proposed methods, and vice-versa, is underlined.

Overall, the stochastic methods of density ratio estimation proposed in this work achieved lower NRMSE than the previously proposed methods ULSIF, KLIEP, and KMM. Observe that, from a total of $3 \times 7=21$ "races" for the best result, the methods proposed in this work achieved the best results in 19 cases $(\approx 90 \%)$, against 2 cases $(\approx 10 \%)$ for the previously proposed methods - KLIEP on model 5, $m=100$ and 200. Moreover, in $11(\approx 48 \%$ ) of the 21 cases, all of the proposed methods obtained results which are better than the ones from the three previously proposed methods - observe that this number, i.e underlined NRMSE entries, is zero for the previously proposed methods. Also note 


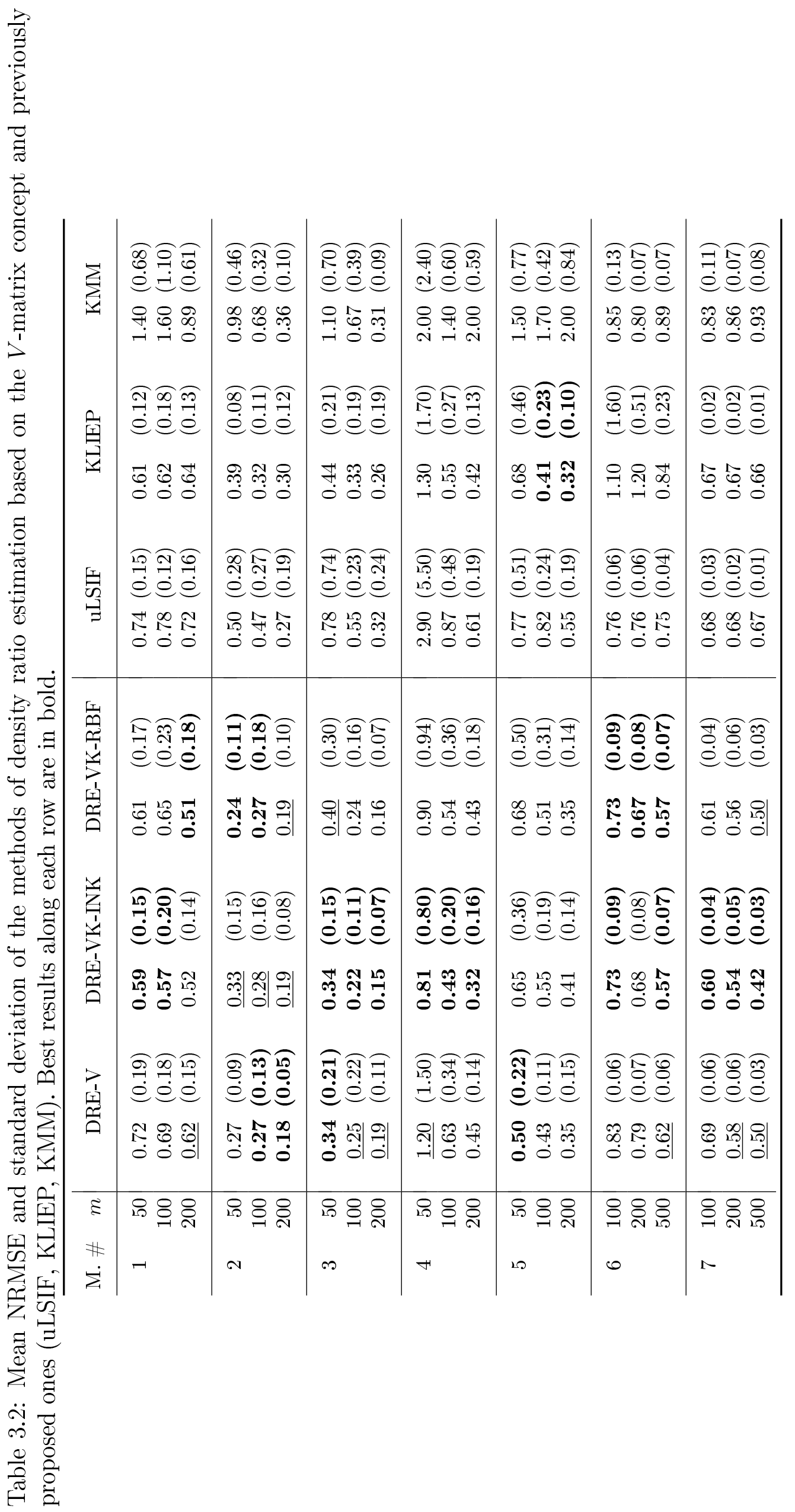


that DRE-VK obtained the best results for Model 1, which was expected as the density ratio from this model cannot be estimated using the other estimators.

Among the methods proposed in this work, from the 21 cases, DRE-V obtained the best results in 4 cases, DRE-VK-INK in 13 cases and DRE-VK-RBF in 6 cases. Observe that there are four ties. Thus, the methods providing smooth estimates in an RKHS (DRE-VK- ${ }^{*}$ ) performed better than DRE-V. It is worth noting that the use of the INKsplines kernel provided equally good or better estimates than the ones provided by the RBF kernel.

We believe that the advantage in accuracy of the methods proposed in this work is due to:

- the flexibility of the stochastic density ratio estimation approach, which allows the estimation of density ratios that cannot be dealt with by previous methods;

- the information provided by the $V$-matrices about the geometry of the data; and

- the smoothness requirements introduced by the RKHS.

\subsection{Final Remarks}

In this chapter, we described several ways of instantiating the three entities of the stochastic density ratio estimation approach - Expression (3.2). We used a sufficient number of instantiations to derive two new methods - DRE-V and DRE-VK. These methods are shown to perform better than previous methods of density ratio estimation. The results using these straightforward instantiations are already very promising for the stochastic density ratio estimation approach.

Using different combinations of $\rho_{2}\left(A_{n} r, F_{1, \ell}\right), \Omega(r)$, and procedures for $\gamma$ selection, one could derive several other methods of stochastic density ratio estimation. Moreover, we could combine individual entities of the same category to form new entities. For instance, we could mix different $\gamma$ selection strategies, or use a regularizer which is a weighted sum of more than one regularizer.

The benefits of more advanced instantiations should always be weighted against the trouble of an increased computational cost. This trade-off is to be explored inside each application of density ratio estimation, as there is no right answer as to what should be privileged in the general problem. 


\section{Chapter 4}

\section{Mutual Information Estimation and Feature Selection}

As mentioned in Chapter 1, one of the applications of density ratio estimation is mutual information estimation. The mutual information between two random vectors $X$ and $Y$ is one of the most important concepts in Information Theory (Cover and Thomas, 2006). For continuous $X$ and $Y$, it is often written as

$$
I(X, Y)=\int_{X} \int_{Y} p(x, y) \log \frac{p(x, y)}{p(x) p(y)} d x d y
$$

where $p(x, y)$ is the joint probability density function of $X$ and $Y$, and $p(x)$ and $p(y)$ are the marginal density functions associated with $X$ and $Y$ (resp.). Intuitively, mutual information measures how much information is shared by $X$ and $Y$ : if they are independent of each other, $p(x, y)=p(x) p(y)$ and then $I(X, Y)=0$. On the other hand, if $X$ and $Y$ are the same random vector, the value of the mutual information achieves its upper bound - the differential entropy of $X$ (or $Y)$.

Mutual information plays an important role in data mining tasks like feature selection (Guyon and Elisseeff, 2003) and Independent Component Analysis (Hyvärinen and Oja, 2000). For these tasks, it is typical for the distributions involved in MI calculation to be unknown. This way, it becomes important to develop methods of mutual information estimation using data sampled from these unknown distributions (van Hulle, 2005; Kraskov et al., 2004; Suzuki et al., 2009).

When $Y$ is a categorical variable, the estimation of the mutual information essentially depends on the estimation of a finite number of ratios of probability densities (Sugiyama et al., 2011; Vapnik et al., 2014). Previous work has already attempted to cast MI estimation as density ratio estimation (Suzuki et al., 2009). However, the approach taken in this work is new. We consider an estimator - namely VMI - that differs from previously proposed estimators in two aspects. The first one is in the form of the MI estimator itself, 
which is more robust. The other one is related to the method of density ratio estimation employed in VMI, which is based on the stochastic approach described in Chapters 2 and 3. We find experimentally that this new MI estimator performs better than previous ones.

This chapter is organized as follows. Section 4.1 describes the approach to mutual information estimation taken in this work as well as previous approaches. Section 4.2 covers a practical feature selection scheme based on the proposed mutual information estimation approach. Section 4.3 is devoted to experimental evaluations and comparisons. Section 4.4 concludes with the findings of this chapter and indications of future research.

\subsection{Mutual Information Estimation}

In this section, we show how mutual information is derived from the definitions of entropy and conditional entropy. After that, we propose a new estimator of mutual information.

\subsubsection{Definition of Mutual Information}

Let $X$ and $Y$ be each a continuous random variable. The value

$$
H(Y)=-\int_{Y} p(y) \log p(y) d y
$$

is called the entropy of $Y$. Additionally, let us consider

$$
p(y \mid x)=\frac{p(x, y)}{p(x)}
$$

which is the conditional density of $Y$ given $X$. The value

$$
H(Y \mid x)=-\int_{Y} p(y \mid x) \log p(y \mid x) d y
$$

is called the conditional entropy of $Y$ given $X=x$. The amount of uncertainty remaining about $Y$ when $X=x$ is given by

$$
\Delta H(Y \mid x)=H(Y)-H(Y \mid x)
$$

Analogously, let us denote by $\Delta H(X \mid y)$ the amount of uncertainty remaining about $X$ when $Y=y$. It turns out that the expectation of each of these variables

$$
\begin{aligned}
& H(Y \mid X)=E_{X}[\Delta H(Y \mid x)] \\
& H(X \mid Y)=E_{Y}[\Delta H(X \mid y)]
\end{aligned}
$$


is useful in defining the mutual information of $X$ and $Y$ :

$$
H(Y)-H(Y \mid X)=H(X)-H(X \mid Y)=I(X, Y)
$$

where $I(X, Y)$ is given by Expression 4.1. In other words, the amount of information shared by $X$ and $Y$ equals the average reduction in uncertainty about $Y$ when $X$ is known, which also equals the average reduction in uncertainty about $X$ when $Y$ is known.

Expression (4.2) explicits some properties of mutual information. First, it follows that $0 \leq I(X, Y) \leq H(Y)$ and $0 \leq I(X, Y) \leq H(X)$. Also, $I(X, Y)=0$ implies $H(Y)=$ $H(Y \mid X)$ and $H(X)=H(X \mid Y)$, i.e knowing $X$ does not reduce the uncertainty about $Y$ and vice-versa. Conversely, $I(X, Y)=H(Y)=H(X)$ implies $H(Y \mid X)=H(X \mid Y)=0$, i.e $Y$ follows deterministically from $X$ and vice-versa.

\subsubsection{Estimators of Mutual Information}

The estimation of the mutual information $I(X, Y)$ based on a sample

$$
\left(x_{1}, y_{1}\right), \ldots,\left(x_{n}, y_{n}\right) \stackrel{i . i . d}{\sim} p(x, y)
$$

is a long-standing problem in applied statistics. Several attempts to solve this problem have been made by considering the equality

$$
I(X, Y)=H(X)+H(Y)-H(X, Y)
$$

That is, $I(X, Y)$ is estimated by first estimating the differential entropies $H(X), H(Y)$, and $H(X, Y)$, and then plugging these estimates into Expression (4.3). A potential problem with the entropy approach is that the errors in the estimation of the individual entropies do not necessarily cancel out.

Two popular entropy estimators are the non-parametric $k$-NN estimator (Kraskov et al., 2004) and the parametric Edgeworth-expansion estimator (van Hulle, 2005). These estimators have some drawbacks of their own. To wit, there is no systematic way of selecting the best value of $k$ in the $k$-NN estimator. Moreover, the Edgeworth estimator is based on the assumption that the densities $p(x, y), p(x)$, and $p(y)$ are each normally distributed, which is often not satisfied in practice.

Motivated by the problems of these entropy estimators and the indirect nature of MI estimation through entropy estimation, a direct estimator of $I(X, Y)$ was proposed in (Suzuki et al., 2009). Using the law of large numbers, the expectation in Expression (4.1) can be replaced by the empirical average to provide the estimator

$$
\widehat{I}(X, Y)=\frac{1}{n} \sum_{i=1}^{n} \log \frac{p\left(x_{i}, y_{i}\right)}{p\left(x_{i}\right) p\left(y_{i}\right)}
$$


Since the value of the ratio inside the log function is unknown, the authors proposed the KLIEP method (Sugiyama et al., 2008) for estimating this ratio using a sample drawn from $p(x, y)$. The resulting estimator was named Maximum Likelihood Mutual Information MLMI.

Here, we put forward a different direct approach to mutual information estimation. For the case where $Y$ is discrete (classification), we will also stumble upon the unavoidable task of estimating the ratio of two probability densities.

The reader might be wondering why we need yet another MI estimator. The answer is that the estimator in Expression (4.4) will be very susceptible to errors in the estimation of the involved ratios, since $\log (z)$ goes to $-\infty$ very fast when $z \rightarrow 0-$ Figure 4.1. Any estimation error of the ratios that may happen in this direction will be greatly magnified, causing a large error in MI estimation. Fortunately, there is an equivalent formulation for MI (Vapnik et al., 2014) which provides a more robust estimator by considering the better behaved function $z \log (z)-$ Figure 4.1. Even though $z \log (z)$ goes to $\infty$ faster than $\log (z)$ when $z \rightarrow \infty$, the value of $z$ will be bounded from above in MI estimation.

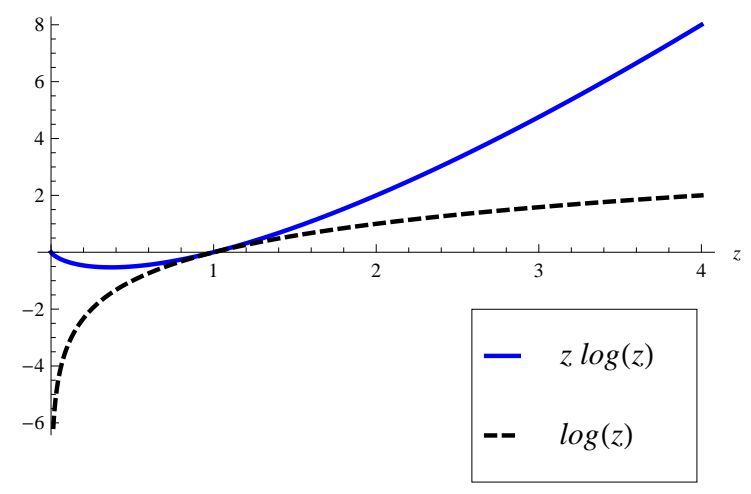

Figure 4.1: Plot of functions $\log (z)$ and $z \log (z)$. In MI estimation, $z$ will be an estimated ratio value. Hence, the estimated value of $z \log (z)$ is less susceptible to estimation errors than $\log (z)$ (see the explanation in the above paragraph).

In order to arrive at this improved estimator, let us first rewrite $I(X, Y)$ as

$$
I(X, Y)=\int_{X} \int_{Y} \frac{p(x, y)}{p(x) p(y)} \log \frac{p(x, y)}{p(x) p(y)} p(x) p(y) d x d y=E_{X} E_{Y}[r(x, y) \log r(x, y)]
$$

where $E$ is the expectation operator and $r(x, y)=\frac{p(x, y)}{p(x) p(y)}$ is a density ratio function. Whenever $Y$ takes only on a finite number of values $\left\{a_{1}, \ldots, a_{m}\right\}$, Expression (4.5) is written as

$$
I(X, Y)=\sum_{i=1}^{m} p\left(a_{i}\right) E_{X}\left[r\left(x, a_{i}\right) \log r\left(x, a_{i}\right)\right]
$$

In this case, $r\left(x, a_{i}\right)=\frac{p\left(x \mid a_{i}\right)}{p(x)}$ can be considered as a density ratio function that depends only on $x$. 
Therefore, the problem of estimating the mutual information in classification settings is equivalent to estimating the value $I(X, Y)$ in Expression (4.6) when the densities $p(x, y)$, $p(x)$, and the probability $p(y)$ are unknown, and a sample $\left(x_{1}, y_{1}\right), \ldots,\left(x_{n}, y_{n}\right) \stackrel{\text { i.i.d }}{\sim} p(x, y)$ is available. Denoting by $n_{i}$ the number of elements from class $a_{i}$ and considering that $n=n_{1}+\ldots+n_{m}$, the probabilities $p\left(a_{i}\right)$ can be readily estimated by $p_{i}=\frac{n_{i}}{n}$. Using the values $p_{i}$, we replace the expectation in Expression (4.6) by its empirical average, arriving at the following estimator for $I(X, Y)$ :

$$
\widehat{I}(X, Y)=\frac{1}{n} \sum_{i=1}^{m} p_{i} \sum_{j=1}^{n} r\left(x_{j}, a_{i}\right) \log r\left(x_{j}, a_{i}\right) .
$$

Once the $n$ values of the $m$ different density ratios $r\left(x_{j}, a_{i}\right)$ are known, consistency of $\widehat{I}(X, Y)$ is guaranteed by the law of large numbers. As these ratios are not known in advance, we use methods of density ratio estimation - Chapter 3. Note that, when only two classes exist, just $n$ ratio values $r\left(x_{1}, a_{1}\right), \ldots, r\left(x_{n}, a_{1}\right)$ need to be estimated, since the other $n$ ratio values $r\left(x_{1}, a_{2}\right), \ldots, r\left(x_{n}, a_{2}\right)$ can be computed from the first ones by applying the law of total probability:

$$
\begin{array}{cc} 
& p\left(x \mid a_{1}\right) p\left(a_{1}\right)+p\left(x \mid a_{2}\right) p\left(a_{2}\right)=p(x) \\
\Leftrightarrow & \frac{p\left(x \mid a_{1}\right)}{p(x)} p_{1}+\frac{p\left(x \mid a_{2}\right)}{p(x)} p_{2}=\frac{p(x)}{p(x)} \\
\Leftrightarrow & r\left(x_{1}, a_{1}\right) p_{1}+r\left(x_{1}, a_{2}\right) p_{2}=1 \\
\Leftrightarrow & r\left(x_{1}, a_{2}\right)=1-\frac{r\left(x_{1}, a_{1}\right) p_{1}}{p_{2}} .
\end{array}
$$

\subsection{Feature Selection Based on Mutual Information}

In this section, we describe how mutual information estimation can be used as a component of a feature selection scheme in classification tasks. Remind that in feature selection the goal is to to describe the training set $\left(x_{1}, y_{1}\right), \ldots,\left(x_{n}, y_{n}\right)$ using only a fraction of the original $d$ features $\left[x_{i}^{1}, \ldots, x_{i}^{d}\right]$. This procedure may suit different purposes, the most common ones being:

- The improvement in classification performance by eliminating irrelevant features;

- The increase in computational efficiency in the classifier training stage.

From the theoretical point of view, this problem breaks down into two stages:

1. Among the original $d$ features, select the $k$ features that provide the largest mutual information towards the target variable $Y$;

2. Train a classifier using the training set restricted to the $k$ selected features. 


\subsubsection{Mutual Information and the Bayes Rule}

Taking mutual information as a criterion of feature set importance is justified for it bounds the probability of erroneous classification of the optimal decision rule - the socalled Bayes function. A higher value of $I(X, Y)$ implies a smaller probability of erroneous classification of the optimal decision rule.

The Bayes function $f^{*}(x)$ is defined as

$$
f^{*}(x)=\underset{y}{\arg \max } p(y \mid x) .
$$

It follows that the probability of erroneous classification of $f^{*}(x)$ is

$$
R^{*}=\int\left[1-\max _{y} p(y \mid x)\right] d x .
$$

Hellman and Raviv (1970) showed that

$$
R^{*} \leq \frac{1}{2} H(Y \mid X)
$$

which implies

$$
R^{*} \leq \frac{1}{2}[H(Y)-I(X, Y)]
$$

From this last expression, it follows that increasing values of $I(X, Y)$ imply smaller probabilities of erroneous classification of the optimal decision rule. When mutual information attains it maximum value, i.e $I(X, Y)=H(Y)$, the probability of erroneous classification of the optimal rule is zero.

To illustrate this relationship, consider Figure 4.2. Each one of the four scatter plots depicts two-class data points sampled from a different artificial two-dimensional data model. Since the model used to generate the data is known, the mutual information $I(X, Y)=I\left(\left[X^{1}, X^{2}\right], Y\right)$ can be calculated in each case. This illustration shows that, as mutual information increases, the clearer is the separation between the points from the two classes.

\subsubsection{Heuristic Solutions to the Theoretical Problem}

Given enough time and data, it is possible to estimate mutual information for each combination of $d$ features taken $k$ at a time and, afterwards, choose the $k$-combination with the largest estimated value of MI. In most cases, however, this procedure is unfeasible. Data is usually scarce, which impairs $k$-dimensional density ratio estimation when $k$ is large. In addition, even if we had enough data for estimating the density ratio for a single combination of $k$ features, the time it would take to run this procedure for all possible 


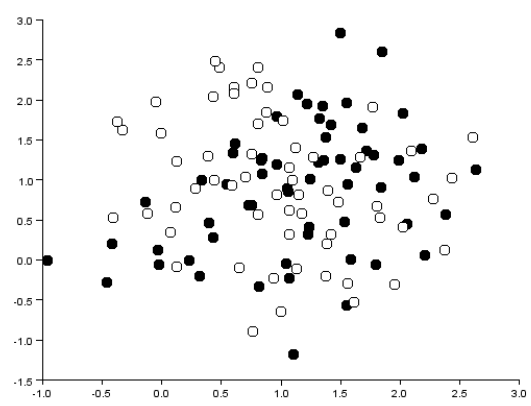

(a) $I(X, Y)=0.10$

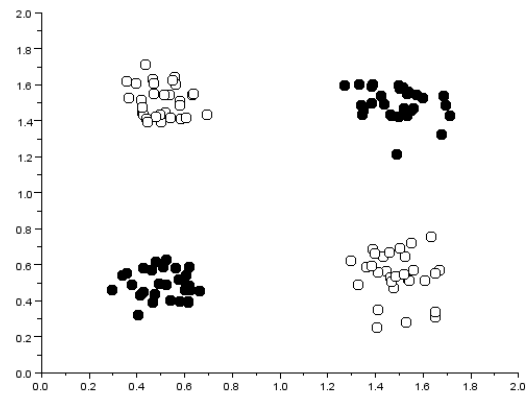

(c) $I(X, Y)=0.99$

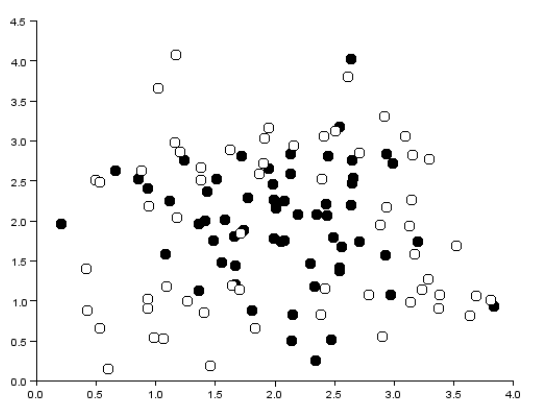

(b) $I(X, Y)=0.30$

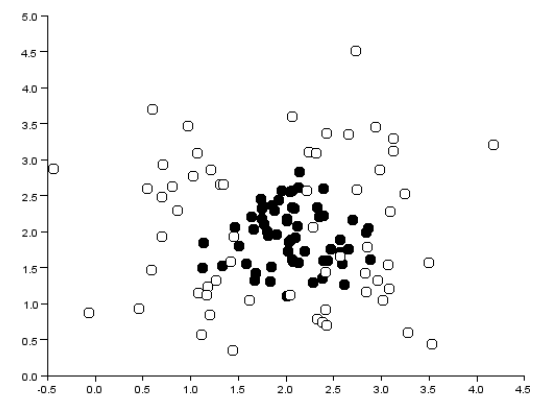

(d) $I(X, Y)=0.56$

Figure 4.2: Mutual information and a data sample of 120 points divided into two classes (circle/solid circle) for different two-dimensional models.

combinations of features is prohibitive for typical ${ }^{1}$ values of $k$ and $d$.

Thus, feature selection is foremost an engineering problem. All sorts of heuristics have been employed over the years to deal with it (Guyon and Elisseeff, 2003). Some of these heuristics have no explicit connection to mutual information. In this work, we employ a heuristic that does not give up on mutual information entirely: it tries to estimate mutual information for low-order combinations of features (say, pairs of features), and, afterwards, selects $k$ features based on these estimates. In (Brown et al., 2012), several methods following this heuristic are investigated. The conclusion was that the Joint Mutual Information (JMI) method (Yang and Moody, 1999) was one of the best methods of this category. Hence, JMI is used in the feature selection experiments described in the next section.

The JMI procedure, which is a kind of forward selection scheme, goes as follows Algorithm 4.1. After the value of mutual information $I\left(\left[X^{i}, X^{j}\right], Y\right)$ is estimated for every pair of features $(i, j)$ using the training set $\left(x_{1}, y_{1}\right), \ldots,\left(x_{n}, y_{n}\right)$, the pair with the largest $\widehat{I}\left(\left[X^{i}, X^{j}\right], Y\right)$ is singled out to compose the initial set of selected features $S$. If $k>2$, the

\footnotetext{
${ }^{1}$ For instance, for $k=4$ and $d=100$, around 4 million combinations need to be investigated. For $k=4$ and $d=1000$, the number goes up to around 400 billion combinations.
} 
method iterates over the remaining features, adding to $S$ the feature $j$ that maximizes

$$
\sum_{i \in S} \widehat{I}\left(\left[X^{i}, X^{j}\right], Y\right)
$$

The running time of this procedure is dominated by the estimation of the mutual information for each pair of features. As datasets with 10 thousand features figure in the experimental evaluation of the next section, the whole procedure would be very time consuming. In these cases only, we rely on one-dimensional mutual information estimation to reduce our working set of features. More precisely, we restrict the application of JMI to the 50 features with the largest value $\widehat{I}\left(X^{i}, Y\right)$.

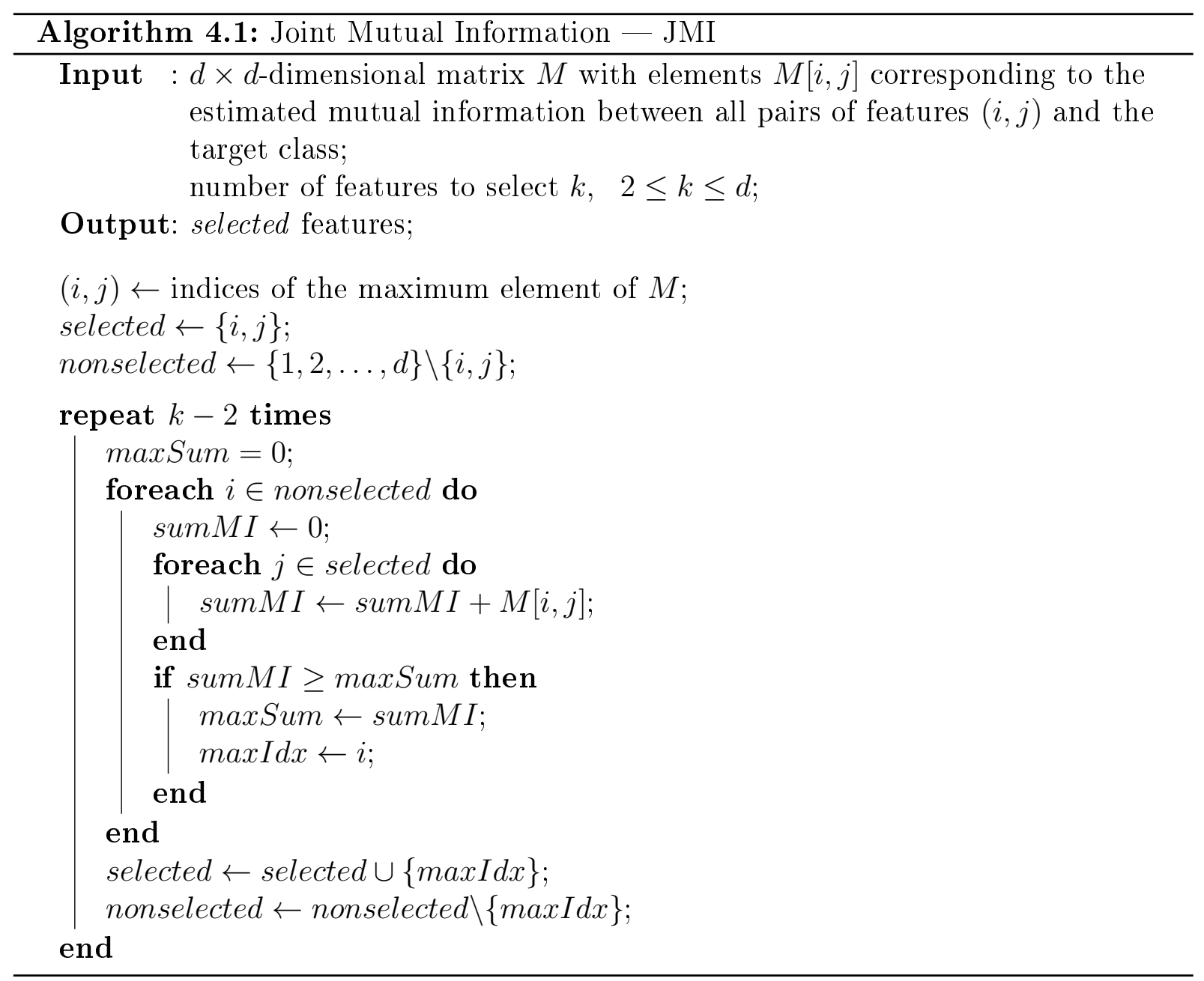

In the MI estimation phase, here we use DRE-V - Section 3.3 - to estimate the density ratios in Expression (4.7). Taking $Y=\{+1,-1\}$ for simplicity, let us assume the set of points $\left\{x_{i_{1}}, \ldots, x_{i_{\ell}}\right\}$ have $y_{i_{1}}=\ldots=y_{i_{\ell}}=+1$. Then, this set of points corresponds to the sample from the numerator density $p(x \mid+1)$, and the complete set of points $x_{1}, \ldots, x_{n}$ corresponds to the sample from the denominator density $p(x)$. When performing $\gamma$ selection through cross-validation, if a point $\left(x_{i},+1\right)$ is held out, it must be held out from both the numerator and denominator samples.

From now on, we employ the name VMI to refer to this method of mutual informa- 
tion estimation. VMI has some advantages over the Edgeworth and $k$-NN estimators, for it is non-parametric and its parameter $\gamma$ can be optimized using the available data. Moreover, VMI uses DRE-V, which was experimentally shown to outperform KLIEP Section $3.5-$, the latter being the estimator used in MLMI.

Algorithm 4.2 shows the general scheme for performing feature selection using VMI and JMI. A MATLAB code for DRE-V is available at

$$
\text { "http://sites.labic.icmc.usp.br/igorab/drev.m". }
$$

This code is tailored to mutual information estimation in binary classification settings and employs leave-one-out cross-validation - Section 3.4.3 - for selecting the parameter of regularization $\gamma$. After $\gamma$ is selected, we obtain the final solution imposing the constraints

$$
\begin{aligned}
& r\left(x_{i}^{\prime}\right) \geq 0, \quad i=1, \ldots, n, \\
& \frac{1}{n} \sum_{i=1}^{n} r\left(x_{i}^{\prime}\right)=1 .
\end{aligned}
$$

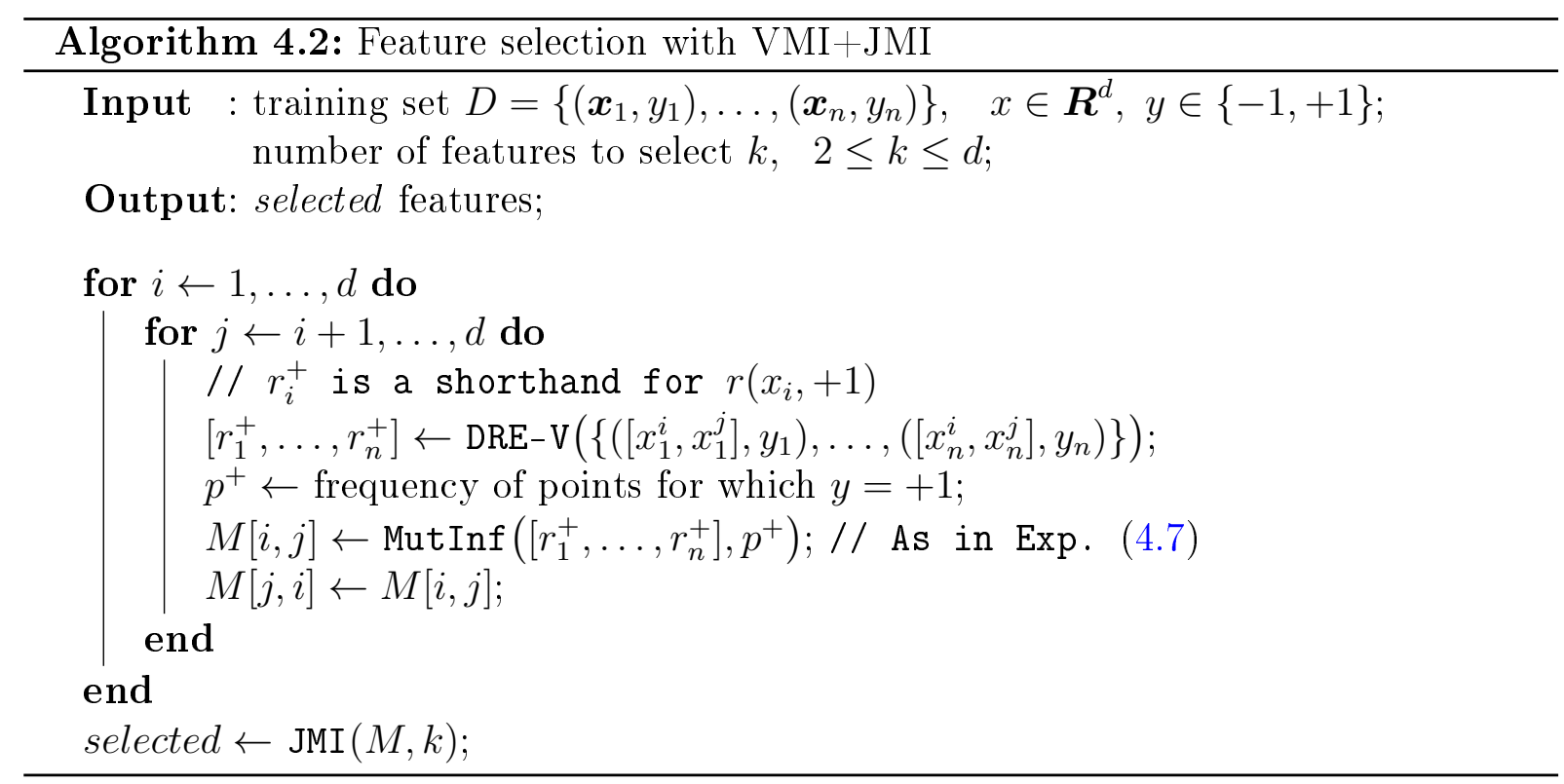

\subsection{Experiments}

In this section, we experimentally evaluate the new approach of mutual information estimation based on both Expression (4.7) and the stochastic density ratio estimation method DRE-V, which we have named VMI. For comparison, we evaluate VMI against the popular $k$-NN and Edgeworth - EDGEW - approaches and the MLMI (Suzuki et al., 2009) estimator.

First, we conducted experiments in 10 synthetic two-dimensional datasets. The 2D estimation case is important, since it provides the basis for a number of existing feature 
selection algorithms, as mentioned in Section 4.2. Using synthetic data allows us to compare the obtained estimates with the real value of mutual information.

A detailed description of these synthetic models is provided in Table 4.1. Illustrative pictures are provided in Appendix A.2. Hereafter, all logarithm calculations are to the base 2 . We consider datasets of size $n=40,100,200$ and 400. For each sample size, 20 different samples are used for estimating $I(X, Y)$. The estimation error $\hat{I}-I_{\text {real }}$ of each method is averaged over these 20 samples.

Table 4.2 reports the results of these experiments. In Table 4.3, we present a summary for each method of how many times their average MI estimation error was the smallest for a given dataset and sample size, and also how many times their average relative error exceeded 50\%. Note that VMI achieves the highest counts in "Best" and the lowest in " $50 \%$ ", with the exception of sample size 40 , where all methods tend to achieve poor results. The MLMI method delivers the worst results in most cases. The Edgeworth estimator starts delivering reasonable results only when the sample size reaches 400 . Finally, the $k$-NN estimators deliver very similar performances for the tested values of $k$. It is worth reminding that, even if the $k$-NN estimator could obtain better performance with a larger value of $k$, it is unknown how to select the best value of $k$ in mutual information estimation.

Now we proceed to the second batch of experiments, this time conducting feature selection in classification tasks. We considered 9 binary classification datasets ${ }^{2}$, and a 10fold cross-validation procedure to generate 10 pairs of training and test sets. Each feature in the training set was normalized to have zero mean and unit variance. The scale factors obtained for the training set were also applied for normalizing the test set. Normalized values greater than 3 or less than -3 were set to 3 and -3 (resp.) in both training and test sets.

The mutual information between each pair of features and the target class was estimated using a fixed mutual information estimation method and the normalized training examples for the dataset. Then, 5 (or 10) features were selected by the JMI procedure described in Section 4.2, and an SVM classifier was obtained from the normalized training set restricted to those selected features. Regarding the parameter selection problem of SVM, we use an RBF kernel where the parameters $C$ and $\sigma$ were selected using the grid-search procedure proposed in Chapter 5.

For comparison purposes, we also ran a widely used feature selection algorithm, ReliefF (Kononenko, 1994; Robnik-Sikonja and Kononenko, 2003), which does not attempt to estimate mutual information. We use the Relief-F implementation of the WEKA framework.

The balanced error rate ${ }^{3}$ of the resulting classifiers was verified on the normalized test set restricted to the 5 (or 10 ) selected features. To add perspective, we also report the

\footnotetext{
${ }^{2}$ Their description is in Table 5.1.

${ }^{3}$ The balanced error gives the same weight to the errors within each class (cf. Section 5.4).
} 
Table 4.1: Synthetic models

\begin{tabular}{|c|c|c|c|c|c|c|}
\hline M. \# & $k$ & $p_{1}$ & $p_{2}$ & $p_{3}$ & $p_{4}$ & $I_{\text {real }}$ \\
\hline 1 & $\begin{array}{l}1 \\
2\end{array}$ & $\begin{array}{l}\operatorname{Beta}(2,2) \\
\operatorname{Beta}(2,2)\end{array}$ & $\begin{array}{l}\text { Uniform }(0,1) \\
\text { Uniform }(0,1)\end{array}$ & - & - & 0.10 \\
\hline 2 & $\begin{array}{l}1 \\
2\end{array}$ & $\begin{array}{l}\text { Uniform }(0,1) \\
\operatorname{Beta}(0.5,0.5)\end{array}$ & $\begin{array}{c}\operatorname{Beta}(0.5,0.5) \\
\operatorname{Beta}(2,2)\end{array}$ & - & - & 0.23 \\
\hline 3 & $\begin{array}{l}1 \\
2\end{array}$ & $\begin{array}{c}\operatorname{Beta}(0.5,0.5) \\
\operatorname{Beta}(2,2)\end{array}$ & $\begin{array}{c}\operatorname{Beta}(1,3) \\
\text { Uniform }(0,1)\end{array}$ & - & - & 0.25 \\
\hline 4 & $\begin{array}{l}1 \\
2\end{array}$ & $\begin{array}{l}\operatorname{Normal}(0.5,1) \\
\operatorname{Normal}(0.5,1)\end{array}$ & $\begin{array}{l}\text { Normal }(1.5,1) \\
\text { Normal }(1.5,1)\end{array}$ & $\begin{array}{l}\operatorname{Normal}(0.5,1) \\
\operatorname{Normal}(1.5,1)\end{array}$ & $\begin{array}{l}\operatorname{Normal}(1.5,1) \\
\operatorname{Normal}(0.5,1)\end{array}$ & 0.03 \\
\hline 5 & $\begin{array}{l}1 \\
2\end{array}$ & $\begin{array}{l}\operatorname{Normal}(0.5,0.5) \\
\text { Normal }(0.5,0.5)\end{array}$ & $\begin{array}{l}\operatorname{Normal}(1.5,0.5) \\
\operatorname{Normal}(1.5,0.5)\end{array}$ & $\begin{array}{l}\text { Normal }(0.5,0.5) \\
\text { Normal }(1.5,0.5)\end{array}$ & $\begin{array}{l}\operatorname{Normal}(1.5,0.5) \\
\operatorname{Normal}(0.5,0.5)\end{array}$ & 0.25 \\
\hline 6 & $\begin{array}{l}1 \\
2\end{array}$ & $\begin{array}{l}\text { Normal }(0.5,0.22) \\
\text { Normal }(0.5,0.22)\end{array}$ & $\begin{array}{l}\operatorname{Normal}(1.5,0.22) \\
\text { Normal }(1.5,0.22)\end{array}$ & $\begin{array}{l}\operatorname{Normal}(0.5,0.22) \\
\operatorname{Normal}(1.5,0.22)\end{array}$ & $\begin{array}{l}\text { Normal }(1.5,0.22) \\
\text { Normal }(0.5,0.22)\end{array}$ & 0.90 \\
\hline 7 & $\begin{array}{l}1 \\
2\end{array}$ & $\begin{array}{l}\text { Normal }(0.5,1) \\
\text { Laplace }(0.5,1)\end{array}$ & $\begin{array}{l}\text { Normal }(1.5,1) \\
\text { Laplace }(1.5,1)\end{array}$ & $\begin{array}{l}\text { Laplace }(0.5,1) \\
\text { Normal }(1.5,1)\end{array}$ & $\begin{array}{l}\text { Laplace }(1.5,1) \\
\text { Normal }(0.5,1)\end{array}$ & 0.07 \\
\hline 8 & $\begin{array}{l}1 \\
2\end{array}$ & $\begin{array}{l}\text { Normal }(0.5,0.5) \\
\text { Laplace }(0.5,0.5)\end{array}$ & $\begin{array}{l}\text { Normal }(1.5,0.5) \\
\text { Laplace }(1.5,0.5)\end{array}$ & $\begin{array}{l}\text { Laplace }(0.5,0.5) \\
\text { Normal }(1.5,0.5)\end{array}$ & $\begin{array}{l}\text { Laplace }(1.5,0.5) \\
\text { Normal }(0.5,0.5)\end{array}$ & 0.67 \\
\hline 9 & $\begin{array}{l}1 \\
2\end{array}$ & $\begin{array}{l}\text { Laplace }(0.5,0.4) \\
\text { Laplace }(0.5,0.4)\end{array}$ & $\begin{array}{l}\text { Uniform }(0,1) \\
\text { Uniform }(0,1)\end{array}$ & - & - & 0.33 \\
\hline 10 & $\begin{array}{l}1 \\
2\end{array}$ & $\begin{array}{c}\text { Laplace }(0.5,0.28) \\
\text { Laplace }(0.5,0.4)\end{array}$ & $\begin{array}{l}\text { Uniform }(0,1) \\
\text { Uniform }(0,1)\end{array}$ & - & - & 0.24 \\
\hline
\end{tabular}

\begin{tabular}{c|c}
\hline$p_{3}$ and $p_{4}$ undefined & $p_{3}$ and $p_{4}$ defined \\
\hline & $p(x)=0.5 \times\left[p_{1}(x)+p_{2}(x)\right]$ \\
& $s(x)=0.5 \times\left[p_{3}(x)+p_{4}(x)\right]$ \\
$q(x)=0.5 \times\left[p_{1}(x)+p_{2}(x)\right]$ & $q(x)=0.5 \times[p(x)+s(x)]$ \\
$r(x)=p_{1}(x) / q(x)$ & $r(x)=p(x) / q(x)$ \\
\hline
\end{tabular}


Table 4.2: Mean and standard deviation of the mutual information estimation error $\hat{I}-$ $I_{\text {real }}$

\begin{tabular}{|c|c|c|c|c|c|c|c|c|}
\hline Model & $I_{\text {real }}$ & $n$ & VMI & MLMI & EDGEW & $3-\mathrm{NN}$ & $5-\mathrm{NN}$ & $7-\mathrm{NN}$ \\
\hline & \multirow{4}{*}{0.10} & 40 & $0.10(0.11)$ & $0.08(0.06)$ & $0.08(0.11)$ & $0.20(0.10)$ & $0.17(0.14)$ & $0.21(0.13)$ \\
\hline \multirow[t]{3}{*}{1} & & 100 & $0.03(0.02)$ & $0.14(0.19)$ & $0.05(0.03)$ & $0.10(0.04)$ & $0.10(0.08)$ & $0.13(0.10)$ \\
\hline & & 200 & $0.03(0.02)$ & $0.10(0.04)$ & $0.04(0.02)$ & $0.08(0.06)$ & $0.07(0.04)$ & $0.05(0.04)$ \\
\hline & & 400 & $0.02(0.01)$ & $0.05(0.02)$ & $0.04(0.01)$ & $0.05(0.04)$ & $0.03(0.03)$ & $0.03(0.03)$ \\
\hline \multirow{4}{*}{2} & \multirow{4}{*}{0.23} & 40 & $0.10(0.07)$ & $0.09(0.09)$ & $0.10(0.05)$ & $0.19(0.18)$ & $0.30(0.13)$ & $0.34(0.16)$ \\
\hline & & 100 & $0.08(0.06)$ & $0.10(0.05)$ & $0.12(0.04)$ & $0.08(0.07)$ & $0.13(0.08)$ & $0.16(0.10)$ \\
\hline & & 200 & $0.05(0.03)$ & $0.11(0.03)$ & $0.12(0.03)$ & $0.08(0.04)$ & $0.09(0.06)$ & $0.10(0.07)$ \\
\hline & & 400 & $0.03(0.02)$ & $0.04(0.04)$ & $0.13(0.01)$ & $0.07(0.04)$ & $0.05(0.04)$ & $0.06(0.05)$ \\
\hline \multirow{4}{*}{3} & \multirow{4}{*}{0.25} & 40 & $0.16(0.11)$ & $0.10(0.08)$ & $0.11(0.11)$ & $0.16(0.13)$ & $0.19(0.12)$ & $0.24(0.14)$ \\
\hline & & 100 & $0.06(0.05)$ & $0.08(0.06)$ & $0.08(0.06)$ & $0.11(0.11)$ & $0.09(0.07)$ & $0.11(0.09)$ \\
\hline & & 200 & $0.04(0.03)$ & $0.06(0.03)$ & $0.05(0.05)$ & $0.05(0.04)$ & $0.08(0.05)$ & $0.08(0.06)$ \\
\hline & & 400 & $0.04(0.03)$ & $0.04(0.03)$ & $0.04(0.04)$ & $0.05(0.04)$ & $0.05(0.03)$ & $0.06(0.04)$ \\
\hline \multirow{4}{*}{4} & \multirow{4}{*}{0.03} & 40 & $0.04(0.05)$ & $0.05(0.05)$ & $0.10(0.09)$ & $0.14(0.12)$ & $0.12(0.10)$ & $0.11(0.10)$ \\
\hline & & 100 & $0.02(0.04)$ & $0.10(0.28)$ & $0.04(0.04)$ & $0.08(0.07)$ & $0.07(0.07)$ & $0.06(0.05)$ \\
\hline & & 200 & $0.02(0.02)$ & $0.06(0.08)$ & $0.04(0.04)$ & $0.07(0.05)$ & $0.05(0.03)$ & $0.05(0.03)$ \\
\hline & & 400 & $0.02(0.01)$ & $0.02(0.01)$ & $0.02(0.01)$ & $0.05(0.05)$ & $0.05(0.04)$ & $0.04(0.03)$ \\
\hline \multirow{4}{*}{5} & \multirow{4}{*}{0.25} & 40 & $0.16(0.10)$ & $0.36(0.60)$ & $0.13(0.16)$ & $0.21(0.16)$ & $0.16(0.11)$ & $0.20(0.12)$ \\
\hline & & 100 & $0.10(0.06)$ & $0.15(0.05)$ & $0.05(0.04)$ & $0.11(0.08)$ & $0.05(0.05)$ & $0.06(0.05)$ \\
\hline & & 200 & $0.07(0.04)$ & $0.10(0.06)$ & $0.05(0.04)$ & $0.07(0.07)$ & $0.07(0.06)$ & $0.06(0.05)$ \\
\hline & & 400 & $0.03(0.02)$ & $0.02(0.02)$ & $0.03(0.02)$ & $0.05(0.04)$ & $0.04(0.03)$ & $0.03(0.03)$ \\
\hline \multirow{4}{*}{6} & \multirow{4}{*}{0.90} & 40 & $0.21(0.31)$ & $0.05(0.05)$ & $0.16(0.11)$ & $0.07(0.04)$ & $0.11(0.10)$ & $0.19(0.20)$ \\
\hline & & 100 & $0.06(0.04)$ & $0.09(0.02)$ & $0.10(0.06)$ & $0.04(0.03)$ & $0.04(0.02)$ & $0.04(0.02)$ \\
\hline & & 200 & $0.04(0.03)$ & $0.05(0.02)$ & $0.04(0.03)$ & $0.04(0.02)$ & $0.04(0.02)$ & $0.04(0.02)$ \\
\hline & & 400 & $0.03(0.02)$ & $0.06(0.04)$ & $0.05(0.04)$ & $0.03(0.02)$ & $0.03(0.02)$ & $0.03(0.02)$ \\
\hline \multirow{4}{*}{7} & \multirow{4}{*}{0.07} & 40 & $0.05(0.04)$ & $0.09(0.04)$ & $0.09(0.09)$ & $0.14(0.10)$ & $0.11(0.10)$ & $0.11(0.10)$ \\
\hline & & 100 & $0.04(0.02)$ & $0.18(0.35)$ & $0.05(0.04)$ & $0.09(0.07)$ & $0.06(0.05)$ & $0.06(0.05)$ \\
\hline & & 200 & $0.03(0.01)$ & $0.08(0.10)$ & $0.04(0.03)$ & $0.05(0.04)$ & $0.05(0.04)$ & $0.05(0.04)$ \\
\hline & & 400 & $0.03(0.01)$ & $0.05(0.03)$ & $0.03(0.03)$ & $0.05(0.04)$ & $0.04(0.03)$ & $0.04(0.02)$ \\
\hline \multirow{4}{*}{8} & \multirow{4}{*}{0.67} & 40 & $0.17(0.17)$ & $0.08(0.07)$ & $0.15(0.16)$ & $0.13(0.09)$ & $0.11(0.10)$ & $0.21(0.14)$ \\
\hline & & 100 & $0.08(0.07)$ & $0.08(0.06)$ & $0.12(0.08)$ & $0.09(0.08)$ & $0.11(0.07)$ & $0.10(0.07)$ \\
\hline & & 200 & $0.06(0.04)$ & $0.03(0.03)$ & $0.08(0.06)$ & $0.08(0.05)$ & $0.07(0.05)$ & $0.07(0.04)$ \\
\hline & & 400 & $0.03(0.03)$ & $0.05(0.02)$ & $0.10(0.04)$ & $0.04(0.03)$ & $0.05(0.03)$ & $0.06(0.03)$ \\
\hline \multirow{4}{*}{9} & \multirow{4}{*}{0.33} & 40 & $0.27(0.06)$ & $0.38(0.37)$ & $0.20(0.13)$ & $0.32(0.20)$ & $0.39(0.16)$ & $0.43(0.13)$ \\
\hline & & 100 & $0.15(0.08)$ & $0.23(0.08)$ & $0.20(0.26)$ & $0.19(0.09)$ & $0.24(0.10)$ & $0.26(0.07)$ \\
\hline & & 200 & $0.12(0.04)$ & $0.10(0.09)$ & $0.15(0.11)$ & $0.14(0.09)$ & $0.16(0.07)$ & $0.19(0.05)$ \\
\hline & & 400 & $0.10(0.02)$ & $0.25(0.04)$ & $0.08(0.09)$ & $0.11(0.05)$ & $0.11(0.05)$ & $0.12(0.05)$ \\
\hline \multirow{4}{*}{10} & \multirow{4}{*}{0.24} & 40 & $0.19(0.05)$ & $0.31(0.51)$ & $0.20(0.06)$ & $0.32(0.21)$ & $0.31(0.15)$ & $0.38(0.17)$ \\
\hline & & 100 & $0.16(0.06)$ & $0.23(0.04)$ & $0.21(0.05)$ & $0.20(0.08)$ & $0.23(0.09)$ & $0.22(0.07)$ \\
\hline & & 200 & $0.09(0.05)$ & $0.19(0.08)$ & $0.19(0.03)$ & $0.11(0.07)$ & $0.14(0.08)$ & $0.16(0.08)$ \\
\hline & & 400 & $0.06(0.03)$ & $0.23(0.05)$ & $0.18(0.01)$ & $0.08(0.04)$ & $0.10(0.05)$ & $0.11(0.04)$ \\
\hline
\end{tabular}

Table 4.3: (Best) Count of datasets in which each method achieved the smallest error. $(>50 \%)$ Count of datasets in which each method achieved $\frac{\hat{I}-I_{\text {real }}}{I_{\text {real }}}>50 \%$.

\begin{tabular}{rr|cccccc}
\hline & $n$ & VMI & MLMI & EDGEW & 3-NN & 5-NN & 7-NN \\
\hline Best & 40 & 3 & 5 & 3 & 0 & 0 & 0 \\
& 100 & 8 & 1 & 1 & 2 & 2 & 1 \\
& 200 & 7 & 2 & 2 & 1 & 1 & 1 \\
& 400 & 8 & 2 & 5 & 1 & 1 & 1 \\
\hline$>50 \%$ & 40 & 6 & 5 & 5 & 6 & 6 & 6 \\
& 100 & 3 & 6 & 5 & 4 & 4 & 4 \\
& 200 & 1 & 4 & 4 & 2 & 4 & 4 \\
& 400 & 1 & 5 & 3 & 2 & 2 & 2 \\
\hline
\end{tabular}


balanced error rate for the classifier obtained on all features.

Here, the application of the $k$-NN estimator and the Relief-F algorithm is distinct from the other methods in that $k$ is treated as just another parameter to be selected in the parameter selection procedure for SVM. This way, we optimized the parameter $k$ directly for feature selection. We could have applied the same procedure to estimate the parameters of MLMI and VMI, yet we chose not to do so in order to evaluate whether better mutual information estimation leads to better feature selection.

The full set of results is presented in Table 4.4. By ordering the mutual information estimators from the lowest (best) balanced error to the highest (worst) one in each row of the table, we arrive at the following average ranking for the methods: 2.3 for $k$-NN, 2.7 for VMI, 2.7 for Relief-F, 3.1 for MLMI, and 4.3 for EDGEW. This ranking puts the feature selection scheme using the $k$-NN estimator as the best one. This result alone should come with no surprise, since the parameter $k$ is being optimized directly to feature selection. Also, notice that the best performing method for a fixed dataset often varies.

Now we focus on those cases that rely only on mutual information estimation, that is, VMI, MLMI, and EDGEW. It is clear that the Edgeworth estimator leads to the worst classification results. According to the average ranking, VMI has a slight advantage over MLMI. However, the average ranking discards the magnitude of the differences in classification error. This way, let us compare the results of VMI and MLMI where the largest discrepancies occurred: in favor of VMI, IONOSPHERE and BIODEG; and in favor of MLMI, SONAR. Notice that the discrepancy in performance when VMI loses is not as large as the opposite. Thus, VMI led to safer feature selection than MLMI.

Comparing VMI to Relief-F, the same conclusions from the previous paragraph apply. The discrepancy in performance when VMI loses is not as large as the opposite. This is especially true for the high dimensional datasets: ARCENE, LUNG-UTERUS, and OvARYKIDNEY. 


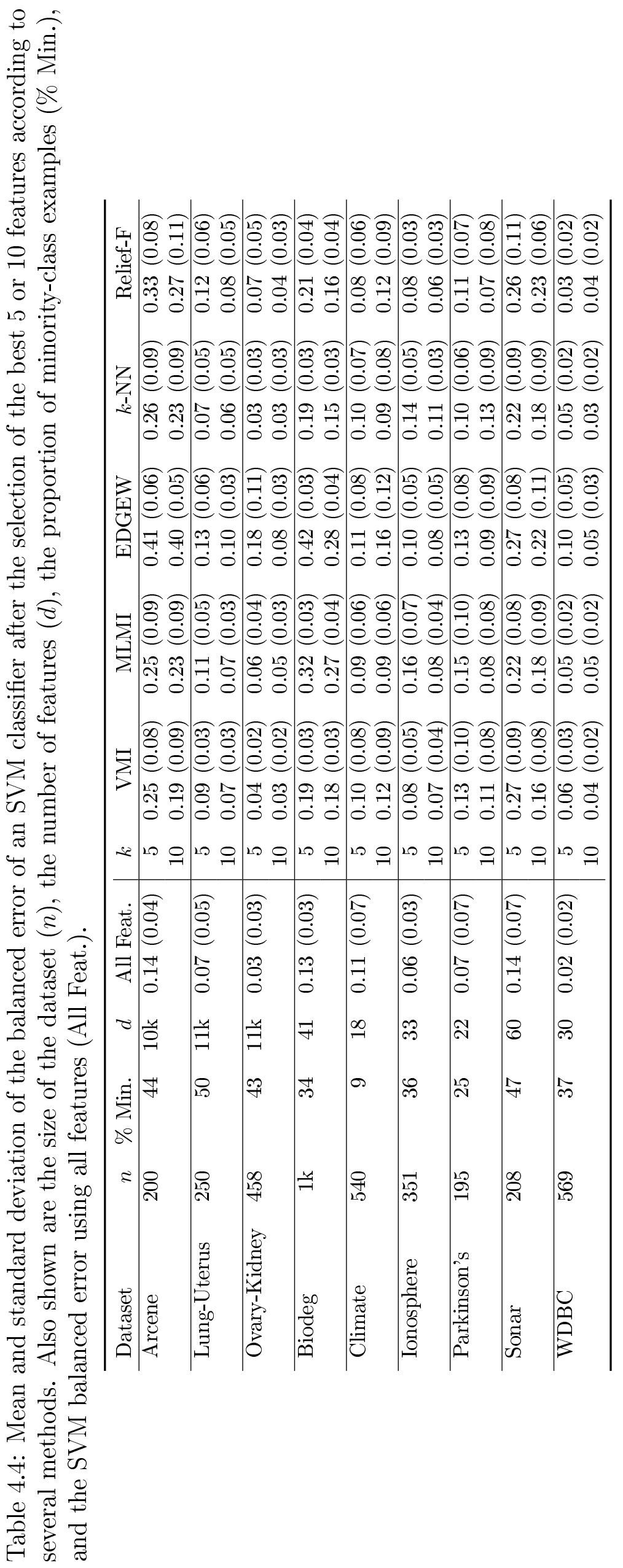




\subsection{Final Remarks}

In this chapter, we investigated VMI, a new mutual information estimation method that was observed in experiments to be more accurate than previous estimators like MLMI, Edgeworth, and $k$-NN. VMI employs the new density ratio estimator DRE-V, developed in Chapter 3. In terms of computational complexity, VMI runs faster than MLMI; the latter is a convex optimization problem while the former is a quadratic optimization problem.

Along with the aforementioned observations, experiments that employed MI estimation for feature selection evidenced that better feature selection can be achieved by using VMI instead of MLMI or Edgeworth. VMI also outperformed the popular feature selection algorithm Relief-F in terms of accuracy of the generated classifiers.

These results allows us to conclude that better mutual information estimation is a way to achieve better feature selection. However, as the experiments with the $k$-NN estimator indicate, it is not the only way: by optimizing the parameter $k$ directly to feature selection, the $k$-NN estimator obtained the best results among the four investigated methods. As the $k$-NN estimator was not the best mutual information estimator in our experiments, these results point to a class of feature selection methods that rely on the identification of feature sets that have a large value of mutual information towards the target class. This identification task comprises another research line on feature selection, as it is different from (and may be simpler than) the estimation of the value of MI. In spite of this, the mutual information estimation scheme provides more details on the importance of features, which allows the design of better selection algorithms than JMI.

For now, we are left with the question: by considering better mutual information estimation, can we hope to construct state-of-the-art methods of feature selection? By using a more advanced density ratio estimation method, it is possible to achieve better mutual information estimation using the approach taken in this chapter. Taking into account that the particular estimator used in our experiments is the simplest instance of the stochastic density ratio estimation method, exploring more sophisticated instances is a good opportunity to verify this question. 


\section{Chapter 5}

\section{Economic Parameter Selection for SVM}

Support Vector Machines (Cortes and Vapnik, 1995) is a powerful and principled machine learning method for data classification. It is rooted in the theory of statistical learning (Vapnik, 1998) and, as such, controls the two factors responsible for generalization: 1) training error and 2) capacity of the set of admissible functions. In order to achieve a higher degree of generalization, both factors should be minimized.

The SVM method has two user-specified parameters that impact the factors mentioned above. One of these parameters is the kernel function, which specifies the set of functions used for data separation. The other one is the generalization parameter $C$, which controls the relative minimization of the training error to the capacity of the learning machine. A small value of $C$ prioritizes the minimization of the capacity at the expense of the training error, whereas a large value of $C$ has the opposite effect.

While conducting the feature selection experiments in Chapter 4, we had to deal with the burden brought by the choice of SVM parameters. As a result, we spotted the opportunity to develop easy-to-follow procedures of parameter selection that can be employed for obtaining good SVM classifiers while retaining computational feasibility. This is in contrast to the unreliable procedure of using "default" parameters or yet the expensive procedure of searching through a large number of parameter candidates, both of which being very common in current SVM usage.

This chapter is organized as follows. Section 5.1 reviews the parameters that affect the generalization ability of Support Vector Machines and the issues that arise when trying to select them. Section 5.2 tackles the selection of the generalization parameter $C$, and presents a new approach for framing this parameter. Section 5.3 deals with the selection of a kernel function for SVM, and presents an easy way for framing the parameter $\sigma$ of RBF kernels. Moreover, a new kind of parameterless kernel function, namely the additive min kernel, is presented. Section 5.4 is devoted to the experimental analysis of the proposed procedures. Section 5.5 concludes advocating the usage of the min kernel for economic SVM classification. 


\subsection{The Problem of Parameter Selection in SVM}

This section is not intended to be a tutorial on SVM classification, as good references on the subject already exist (Burges, 1998; Vapnik, 1998; Cristianini and Shawe-Taylor, 2000). Besides the parameters discussed in this section, there may be other parameters related to specific SVM solvers, though they are more important from a numerical rather than a generalization perspective.

\subsubsection{SVM and its Parameters}

Given $n$ training points $\left(\boldsymbol{x}_{1}, y_{1}\right), \ldots,\left(\boldsymbol{x}_{n}, y_{n}\right)$, where $\boldsymbol{x} \in \boldsymbol{R}^{d}$ and $y \in\{+1,-1\}$, the optimization problem that emerges from SVM is usually expressed in its dual form:

$$
\begin{array}{cl}
\underset{\alpha_{1}, \ldots, \alpha_{n}}{\max } & \sum_{i=1}^{n} \alpha_{i}-\frac{1}{2} \sum_{i=1}^{n} \sum_{j=1}^{n} \alpha_{i} \alpha_{j} y_{i} y_{j} k\left(\boldsymbol{x}_{i}, \boldsymbol{x}_{j}\right) \\
\text { subject to } & \sum_{i=1}^{n} \alpha_{i} y_{i}=0, \\
& 0 \leq \alpha_{i} \leq C .
\end{array}
$$

In this quadratic optimization problem, the constant $C>0$ and the kernel function $k: \boldsymbol{R}^{d} \times \boldsymbol{R}^{d} \mapsto \boldsymbol{R}$ are parameters to be defined. After solving the problem for a fixed $C$ and $k$, the output variables $\alpha_{i}$ are used to calculate the function values $f(\boldsymbol{x})$ used to classify unseen data. Let us define $w(\boldsymbol{x})$ as

$$
w(\boldsymbol{x})=\sum_{i=1}^{n} \alpha_{i} y_{i} k\left(\boldsymbol{x}, \boldsymbol{x}_{i}\right)
$$

and $b$ as

$$
b=-\frac{\max _{y_{i}=-1} w\left(\boldsymbol{x}_{i}\right)+\min _{y_{i}=+1} w\left(\boldsymbol{x}_{i}\right)}{2}, \quad\left\{i: 0<\alpha_{i}<C\right\} .
$$

Then, the class of a point $\boldsymbol{x}$ is computed as

$$
f(\boldsymbol{x})=\operatorname{sign}(w(\boldsymbol{x})+b)
$$

We start by analyzing the so-called generalization parameter $C$. When a learning algorithm uses training data to obtain a predictive function $f(\boldsymbol{x})$ from a set of functions $\mathcal{F}$, it does so by means of an inductive principle. In the case of SVM, induction is performed by the Structural Risk Minimization principle (Vapnik, 1998, Section 6), which controls the number of training errors made by $f(\boldsymbol{x})$ and the capacity (diversity) of the set of functions $\mathcal{F}$. In order to obtain a function $f(\boldsymbol{x})$ that delivers good classification performance on test points, both factors should be small. As the simultaneous minimization of these two factors is contradictive, a balance has to be found by controlling the parameter $C$. 
The effect of $C$ in SVM is clearer from its primal optimization problem, which for simplicity we show for the linear kernel case:

$$
\begin{array}{cl}
\min _{\boldsymbol{w}, b, \xi_{1}, \ldots, \xi_{n}} & \|\boldsymbol{w}\|^{2}+C \sum_{i=1}^{n} \xi_{i} \\
\text { subject to } & y_{i}\left(\boldsymbol{w} \cdot \boldsymbol{x}_{i}+b\right) \geq 1-\xi_{i}, \\
& \xi_{i} \geq 0 .
\end{array}
$$

Note that large values of $C$ put more emphasis on the minimization of the slack variables $\xi_{i}$, which leads to correct separation of the training points, yet risking overfitting. On the other hand, small values of $C$ put more emphasis on the minimization of $\|\boldsymbol{w}\|$, which leads to the simultaneous maximization of the separating margin and minimization of the capacity of the set of functions being considered (Vapnik, 1998, Section 10). This case, however, may lead to underfitting. Figure 5.1 illustrates this trade-off.
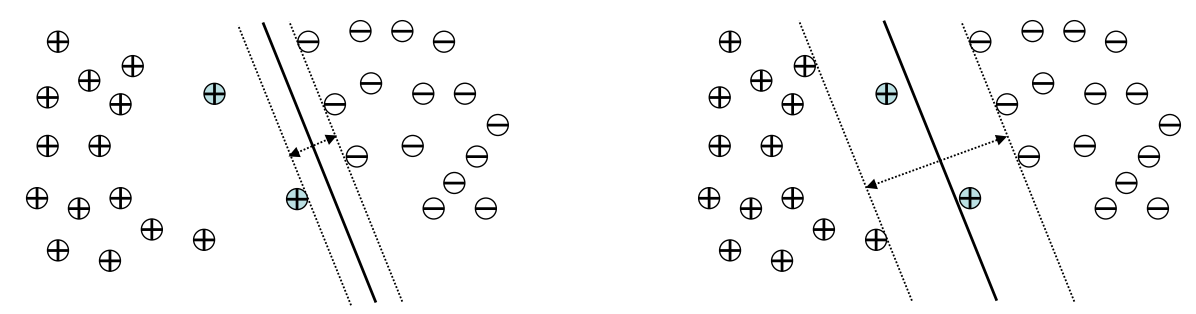

Figure 5.1: Typical linear SVM scenario. The value of $C$ used on the left is larger than the one used on the right.

We now turn our attention to the kernel function parameter $k$, which induces the set of functions $\mathcal{F}_{K}$ on which capacity control is applied. Remind that the kernel function value $k(\boldsymbol{x}, \boldsymbol{z})$ calculates the inner product $\langle\boldsymbol{x}, \boldsymbol{z}\rangle_{K}$ in a transformed space. Two kinds of kernel functions have been extensively employed: the Gaussian RBF and the linear types. To illustrate the effect of selecting a kernel function, we base the following explanation on RBF kernels.

Indeed, Gaussian RBF kernels form a family of kernel functions parameterized by $\sigma>0$ and with values

$$
k_{r b f}(\boldsymbol{x}, \boldsymbol{z})=\exp \left(-\sigma\|\boldsymbol{x}-\boldsymbol{z}\|_{2}^{2}\right)
$$

This means that by choosing $\sigma$, we are effectively choosing a kernel function for SVM. The effect of choosing increasingly larger values of $\sigma$ is to make the kernel an identity evaluator, that is

$$
\begin{aligned}
& k_{r b f}(\boldsymbol{x}, \boldsymbol{z})=1, \text { if } \boldsymbol{x}=\boldsymbol{z}, \\
& k_{r b f}(\boldsymbol{x}, \boldsymbol{z}) \approx 0, \text { if } \boldsymbol{x} \neq \boldsymbol{z} .
\end{aligned}
$$

On the other hand, choosing very small values of $\sigma$ has the effect of making $k_{r b f}(\boldsymbol{x}, \boldsymbol{z}) \approx 1$ for any $\boldsymbol{x}$ and $\boldsymbol{z}$. Figure 5.2 illustrates the effect of $\sigma$ in the SVM decision function $f(\boldsymbol{x})$. 

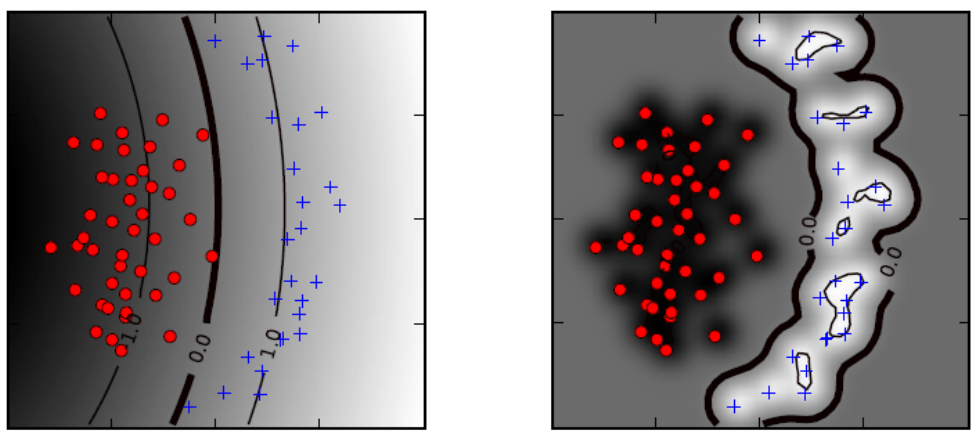

Figure 5.2: SVM decision function using Gaussian RBF kernels. The value of $\sigma$ used on the left is smaller than the one used on the right. (Source: Ben-Hur and Weston (2010))

Note how a large value of $\sigma$ allows for more diversity in the set of functions $\mathcal{F}_{K}$, i.e any training point will be correctly separated at the expense of an increased risk of overfitting.

In summary, the idea of parameter selection in SVM is to select a kernel $k$ and a $C>0$ such that the obtained SVM function $f(\boldsymbol{x}), f(\boldsymbol{x}) \in \mathcal{F} \subseteq \mathcal{F}_{K}$, may separate the training points with small error while belonging to a set $\mathcal{F}$ with small capacity.

\subsubsection{Current Selection Strategies}

As the parameter selection process in SVM directly impacts the generalization ability of the obtained classifier, doing it properly is a vital step when using SVM. This particular characteristic of SVM can be a bless or a curse. It can be a bless because we can push the process towards better classification; and a curse because current general approaches for dealing with it are either unreliable or computationally demanding.

An unreliable but efficient approach is to resort on "default" parameters, which would guarantee reasonable performance at a first attempt. However, there is no such thing for SVM. For instance, the best value of $C$, i.e the value $C_{\text {best }}$ that provides the best classification performance on unseen data, varies greatly with the problem at hand and the chosen kernel function (Braga et al., 2013). In its turn, the choice of the kernel function is also problem dependent. One may try some known kernel functions, though this is not a guarantee of reasonable performance.

It is worth noting that quite different default parameters are used in different SVM implementations. For example, in WEKA ${ }^{1}$ (Witten et al., 2010) and LIBSVM $^{2}$ (Chang and Lin, 2011) the default value of $C$ is 1 . In SVMlight ${ }^{3}$ the default value is the average of $\left\langle\boldsymbol{x}_{i}, \boldsymbol{x}_{i}\right\rangle_{K}^{-1}$ for the training points, while in $\mathrm{SVMtorch}^{4}$ the default value is 100 . Moreover, WEKA and SVMlight use the linear kernel as default, while LIBSVM uses RBF.

\footnotetext{
${ }^{1}$ http://www.cs.waikato.ac.nz/ml/weka/

${ }^{2}$ http://www.csie.ntu.edu.tw/ cjlin/libsvm/

${ }^{3}$ http://svmlight.joachims.org/

${ }^{4}$ http: //www.torch.ch/
} 
Although there are some guidelines for choosing a kernel function among the popular ones, which we present in Section 5.3, a computationally easy way of selecting $C$ is mysterious even to SVM experts. The current practice is to call the SVM solver many times, each time with a different candidate value of $\mathrm{C}$. Then, from the analysis of the obtained solutions, one looks for clues to find the classifier with the best predictive performance.

The theoretical approach for assessing the generalization ability of an SVM classifier is to bound its expected error (Vapnik, 1998, Section 10). Existing bounds typically depend on the training error, the margin of the separating classifier, the data, and the kernel function being used. Unfortunately, these bounds tend to be too conservative for practical consideration, so that a validation procedure, like the 10 -fold cross-validation procedure, is usually taken in practice.

In any assessment approach, many candidates of $C$ should be considered. The most important problem with this approach is how to choose the range of values from which the candidates of $C$ are taken. Usually, the starting and end points of this interval are several orders of magnitude apart, ensuring that a good candidate of $C$ exists within the considered range. However, the greater the range, the larger the number of candidates needed to cover it.

Choosing the range and the number of candidates impact the computational performance of parameter selection. If a single call to an SVM solver is moderately expensive, making it many times will eventually render the whole parameter selection procedure unfeasible. This situation happens more often than not. For instance, suppose that we have $\ell$ candidates of $C$ and a $k$-fold cross-validation procedure is conducted. That amounts to $\ell \times k$ calls to the SVM solver. To make things worse, suppose that one among $m$ different kernel functions is to be selected: now $\ell \times k \times m$ calls needs to be made. Going a little bit further, imagine that we are in a multi-class context, and that $c$ classes are possible. Then, the problem is reduced to $c$ binary problems if a one-versus-all strategy is used and to $O\left(c^{2}\right)$ binary problems if a one-versus-one strategy is used, with each of this binary problems having its own independent parameter selection problem.

This reasoning leads to the conclusion that in order to obtain good results with SVM in a computational efficient way, users must carefully select a small set of parameter candidates. If users give up on parameter selection to resort on pretense default values, they may get poor performance from SVM. On the other hand, if users try to perform parameter selection without the knowledge to narrow down the number of choices, it might happen that the parameter selection procedure become unfeasible.

In the next sections, we explore easy-to-follow procedures for parameter selection, which focus on reducing the number of candidates of $C$ and of kernel functions that need to be considered for achieving good classification performance with SVM. 


\subsection{Selecting the Generalization Parameter}

As mentioned in the last section, it is not easy in general to obtain a small range of candidates of $C$. However, computational tractability mandates that only a handful of candidates of $C$ should be considered. While it is difficult to frame $C$ in general, there may be specific situations that allow us to obtain compact ranges.

The driving force of the procedure proposed in this section is to eliminate wasteful calls to an SVM solver. The first fact we use to arrive at this procedure is that very small values of $C$ provide classifiers that tend to underfit. Knowing that, there will be a value $C_{\text {low }}$ such that any value $C<C_{\text {low }}$ provides a classifier with the same expected error than the classifier obtained using $C_{\text {low }}$. If, in addition, $C_{\text {low }}$ is such that it yields a bad classifier, any value of $C$ less than $C_{l o w}$ is useless as a candidate.

The problem is how to calculate $C_{\text {low }}$. In what follows, we describe a situation that allows us to calculate this value. For that, it is useful to obtain a bound on the absolute value of the SVM output function $f(\boldsymbol{x})$.

Proposition 5.1. Let $n$ be the number of training points and $k^{\max }=\max _{1 \leq i \leq n}\left|k\left(\boldsymbol{x}_{i}, \boldsymbol{x}_{i}\right)\right|$. Then, for any $\boldsymbol{x},|f(\boldsymbol{x})|<2 n C k^{\max }$.

Proof. Note that

$$
|f(\boldsymbol{x})|=|w(\boldsymbol{x})+b| \leq|w(\boldsymbol{x})|+|b| .
$$

That is, we need to bound the absolute values of $w(\boldsymbol{x})$ and $b$ to get a bound for $|f(\boldsymbol{x})|$. Using Expression (5.1) along with the facts $\alpha_{i} \leq C-$ constraint in the dual optimization problem of SVM - and $\left|k\left(\boldsymbol{x}, \boldsymbol{x}_{i}\right)\right| \leq k^{\text {max }}$ - positive-definite kernel:

$$
|w(\boldsymbol{x})|=\left|\sum_{i=1}^{n} \alpha_{i} y_{i} k\left(\boldsymbol{x}, \boldsymbol{x}_{i}\right)\right| \leq \sum_{i=1}^{n}\left|\alpha_{i} y_{i} k\left(\boldsymbol{x}, \boldsymbol{x}_{i}\right)\right| \leq \sum_{i=1}^{n} C k^{\max }=n C k^{\max } .
$$

Using Expressions (5.5) and (5.2):

$$
|b|=\left|-\frac{\max _{y_{i}=-1} w\left(\boldsymbol{x}_{i}\right)+\min _{y_{i}=+1} w\left(\boldsymbol{x}_{i}\right)}{2}\right|<\frac{n C k^{\max }+n C k^{\max }}{2}=n C k^{\max } .
$$

Finally, plugging Expressions (5.5) and (5.6) into Expression (5.4):

$$
|f(\boldsymbol{x})| \leq|w(\boldsymbol{x})|+|b|<2 n C k^{\max } .
$$


Proposition 5.2. $C<\frac{1}{2 n k^{m a x}}$ implies $|f(\boldsymbol{x})|<1$.

Proof. Proposition 5.2 follows directly from Proposition 5.1. To guarantee $|f(\boldsymbol{x})|<1$, it suffices to have $2 n C k^{\max }<1$. This means that a good choice of $C_{l o w}$ is to take $C_{\text {low }}=\frac{1}{2 n k^{\text {max }}}$, because taking $C<C_{\text {low }}$ has the effect of not allowing the training points to become a support vector.

So far, the value $C_{\text {low }}$ depends on the calculation of $k^{\max }$. This calculation can be avoided when employing normalized kernels. A normalized kernel $\tilde{k}$ has the value

$$
\tilde{k}(\boldsymbol{x}, \boldsymbol{z})=\frac{k(\boldsymbol{x}, \boldsymbol{z})}{\sqrt{k(\boldsymbol{x}, \boldsymbol{x}) k(\boldsymbol{z}, \boldsymbol{z})}} .
$$

Note that $\tilde{k}_{\max }=\max _{1 \leq i \leq n}\left|\tilde{k}\left(\boldsymbol{x}_{i}, \boldsymbol{x}_{i}\right)\right|=1$. Thus, for normalized kernels:

$$
C_{\text {low }}=\frac{1}{2 n}
$$

It is interesting to verify other formulas for $C_{l o w}$, as Expression 5.8 has the disadvantage of setting $C_{\text {low }}$ inversely proportional to the number $n$ of training points. When $n$ is a large number, the value $C_{\text {low }}$ will be too small, contributing to stretch the range of candidates of $C$. To alleviate this problem, other formulas could be investigated, such as $\frac{1}{2 \sqrt{n}}$ or $\frac{1}{2 \log n}$. Other possibility is to consider a large quantile of the distribution of kernel values, say, $90 \%$, and fixing $C_{l o w}=\frac{1}{2 n k^{90 \%}}$. We experimentally analyze these formulas in Section 5.4.

Now that we have obtained a lower bound for $C$, it begs the question whether an upper bound on $C$ would be useful for restricting the number of candidates of $C$. Such an upper bound exists naturally in SVM when the data is separable given the kernel being employed. It is the value $C_{u p}$ for which any $C>C_{u p}$ yields the very same classifier as using $C_{u p}$. That is, $C_{u p}$ is the value of $C$ that turns the soft-margin SVM into a hardmargin one. In this case, $\alpha_{i}$ will always be strictly smaller than $C>C_{u p}$. Despite the existence of $C_{u p}$, it is not easy to calculate what this number is before running the SVM solver and verifying that $\alpha_{i}<C$ for all $1 \leq i \leq n$, as there is a complicated relationship among $C_{u p}$, the kernel values, and the classification problem at hand.

Even if we could calculate $C_{u p}$, it could be the case that the SVM classifier obtained using it would not achieve good performance. This happens particularly in noisy data sets, where it is not desirable to obtain a classifier with zero training error. In these cases, $C_{\text {best }}$ will be less than $C_{u p}$.

Taking everything into consideration, we propose the following procedure for selecting $C$ in SVM - Algorithm 5.1. We start by assessing the classifier obtained with $C \leftarrow C_{\text {low }}$ and iteratively assess $C \leftarrow C_{\text {low }} \times s$, typically $s=10$, until the classification performance of the current classifier is worse than the last one, or a number of maximum iterations 
have been performed ${ }^{5}$. After the first iterative phase, the procedure enters a second phase in which at most two candidates are assessed in the neighborhood of the best solution found in the first phase. For instance, consider $s=10$. When entering the second phase, if $C_{\text {best }}=a$, then the two candidates to be evaluated are $a \times 10^{0.5}$ and $a \times 10^{-0.5}$.

The procedure described in Algorithm 5.1 has an issue when cross-validation is used as the method of performance assessment. It may happen that the cross-validation error goes up from one iteration to another and, after that, goes down once again. The procedure described in Algorithm 5.1 will not investigate candidates of $C$ past the one which increased the cross-validation error. In Section 5.4, we experimentally investigate whether this problem is of major concern, and, in that case, how to avoid it.

\subsection{Selecting the Kernel Function}

The selection of a kernel function is entirely problem dependent when one tries to obtain the best performance out of SVM. Still, knowing how to select a standard kernel function is important in two ways: first, it provides a baseline for future investigation about better kernel functions for the problem; second, it may provide good enough performance, to the point of dismissing further investigations.

According to common guidelines (Hsu et al., 2003), Gaussian RBF and linear kernels have a prominent role in SVM classification. The linear kernel $k_{\text {lin }}(\boldsymbol{x}, \boldsymbol{z})=\boldsymbol{x} \cdot \boldsymbol{z}$ is preferred over RBF when the number of coordinates (features) $d$ of the training points exceeds the number of training examples $n-$ say, $d$ is more than twice of $n$. Experimental practice shows that the classification performance of SVM when using a linear kernel is slightly better than that of the RBF kernel in high-dimensional cases. In these cases, RBF becomes very unattractive, given the extra parameter to be selected.

As to low-dimensional classification problems, the situation is reversed. In these cases, RBF kernels are more indicated, as the majority of these problems fails to be (approximately) linearly separable. An important property of RBF kernels is their universal function approximation ability (Steinwart, 2001). Polynomial kernels also have this property when the polynomial degree is a free parameter. However, numerical difficulties and the Runge's phenomenon soon arise with an increasing degree, precluding widespread use.

From the point of view of current approaches of SVM parameter selection, the lowdimensional case is at a disadvantage with respect to the high-dimensional one, as RBF contributes to multiply the cost of parameter selection due to the extra parameter. In what follows, we investigate how to perform an economic selection of candidates for the RBF parameter $\sigma$. After that, we describe recent advances in non-linear parameterless kernels, which help to alleviate even more the costs of parameter selection in SVM classification.

\footnotetext{
${ }^{5}$ Additionally, verifying whether $C>C_{u p}$ can help to stop the procedure earlier.
} 


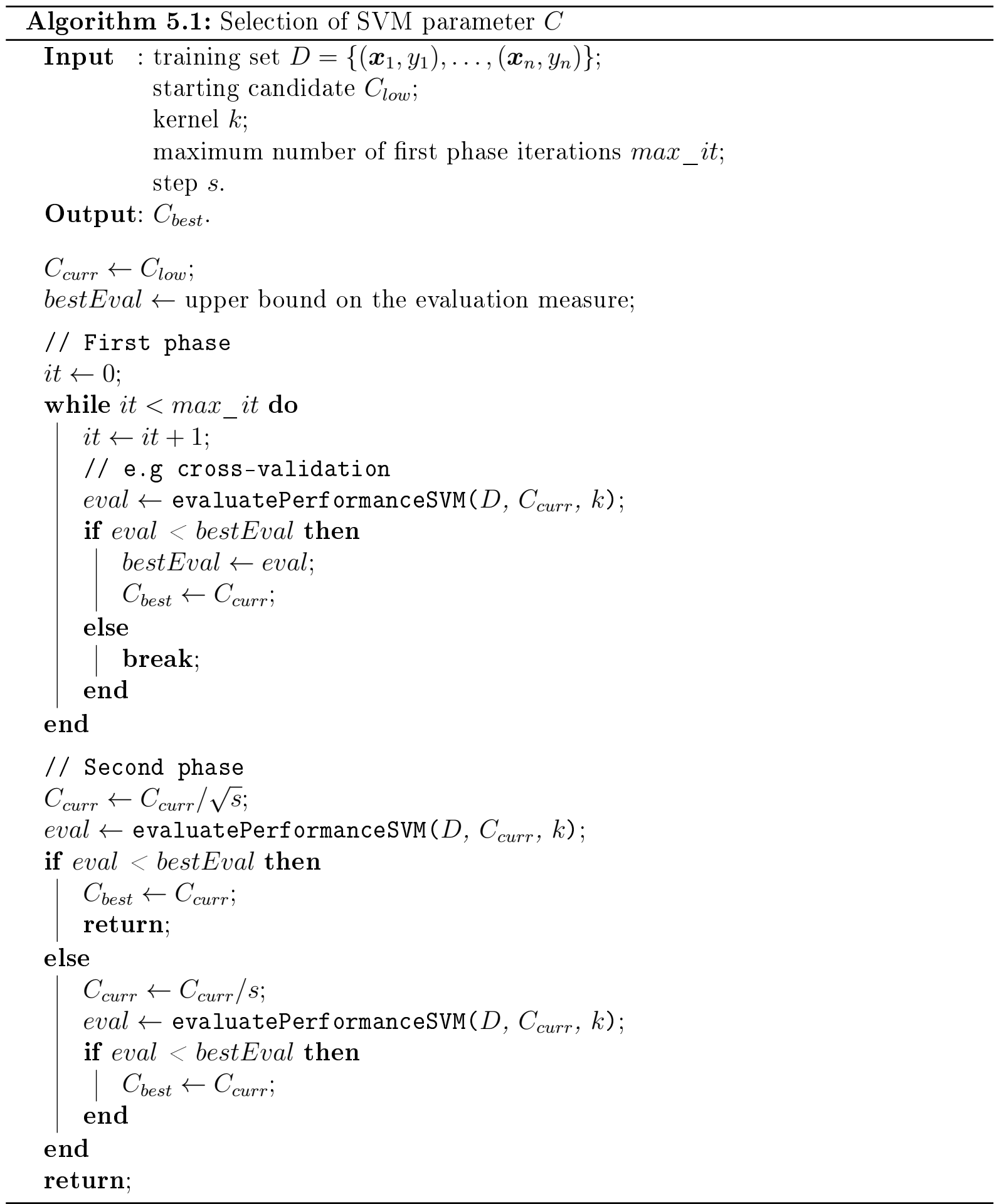




\subsubsection{Selecting the RBF Parameter}

From the parameterized definition of the Gaussian RBF kernel in Expression (5.3), we see that the choice of the parameter $\sigma$ is equivalent to choosing a kernel function. Hence, careful selection of $\sigma$ is important not only due to the number of candidates it generates. In addition, there is an interplay between $\sigma$ and $C$. Remember that when $\sigma$ is small enough, the resulting kernel value $k_{r b f}(\boldsymbol{x}, \boldsymbol{z})$ will tend to 1 no matter what $\boldsymbol{x}$ and $\boldsymbol{z}$ are. In this situation, the kernel matrix will be very ill-conditioned (Fornberg et al., 2004), and the range of candidates of $C$ stretches considerably.

In order to choose candidates of $\sigma$, it is typical to consider the distribution of the values $\rho\left(\boldsymbol{x}_{i}, \boldsymbol{x}_{j}\right)=\left\|\boldsymbol{x}_{i}-\boldsymbol{x}_{j}\right\|_{2}^{2}$. One possible approach is to choose candidates that revolve around the inverse of the mean (or median) value of $\rho\left(\boldsymbol{x}_{i}, \boldsymbol{x}_{j}\right)$. This way, we avoid choosing a too small or too large candidate of $\sigma$. Next, we take advantage of a data pre-processing step to approximate such mean value using a formula, thus avoiding its calculation from data.

When employing SVM classification using RBF kernels, it is very desirable to bring all features of the dataset to the same scale, as features ranging in larger intervals tend to dominate the calculation of distances in the RBF kernel. A popular procedure to tackle the scaling problem is data standardization: for each coordinate (feature) of the training points, the mean and standard deviation values are computed; then, each feature value is subtracted by the corresponding mean and divided by the corresponding standard deviation. The effect of conducting this procedure on data is that every feature will have an average value of 0 and sample variance 1 . By assuming that each feature follows a Normal distribution with mean 0 and variance 1 , we can come up with an approximation for the mean value of $\rho\left(\boldsymbol{x}_{i}, \boldsymbol{x}_{j}\right)$.

Proposition 5.3. Let $\boldsymbol{x}=\left(x^{1}, \ldots, x^{d}\right)$ and $\boldsymbol{z}=\left(z^{1}, \ldots, z^{d}\right)$ be two random vectors such that $x^{j}$ and $z^{j}$ follow a Normal distribution with mean 0 and variance 1 (standard Normal variables). Then the random variable $\rho(\boldsymbol{x}, \boldsymbol{z})=\|\boldsymbol{x}-\boldsymbol{z}\|^{2}$ follows a Gamma distribution with shape parameter $s=\frac{d}{2}$ and scale parameter $\theta=4$.

Proof. It is well known that, if $x^{j}$ and $z^{j}$ are standard normal variables, then the random variable $\left(x^{j}-z^{j}\right)$ follows a Normal distribution with mean 0 and variance 2 . Thus, $\left(\frac{x^{j}-z^{j}}{\sqrt{2}}\right)$ is also a standard normal variable. By definition, the sum of the squares of $d$ independent standard normal variables follows a Chi-squared distribution with $d$ degrees of freedom - $\chi^{2}(d)$. Thus, the random variable $\mathcal{A}=\sum_{j=1}^{d}\left(\frac{x^{j}-z^{j}}{\sqrt{2}}\right)^{2}$ is $\chi^{2}(d)$. Note that

$$
\rho(\boldsymbol{x}, \boldsymbol{z})=\|\boldsymbol{x}-\boldsymbol{z}\|^{2}=\sum_{j=1}^{d}\left(x^{j}-z^{j}\right)^{2}=2 \mathcal{A} .
$$

Moreover, the Chi-squared distribution and the Gamma distribution are related in the 
following way: if $\mathcal{B}$ is $\chi^{2}(\nu)$ and $c>0$, then $c \mathcal{B}$ is distributed according to a Gamma distribution with shape parameter $s=\frac{\nu}{2}$ and scale parameter $\theta=2 c$. As $\rho(\boldsymbol{x}, \boldsymbol{z})=2 \mathcal{A}$ and $\mathcal{A}$ is $\chi^{2}(d)$, it follows that $\rho(\boldsymbol{x}, \boldsymbol{z})$ is distributed according to a Gamma distribution with $s=\frac{d}{2}$ and $\theta=4$.

When a random variable follows a Gamma distribution, its mean value corresponds to the product $s \theta$. Under the conditions stated in the aforementioned proposition, it follows that the mean value of $\rho(\boldsymbol{x}, \boldsymbol{z})$ is $2 d$. Thus, assuming the coordinates of the training points are each standard Normal variables, we expect the average value of $\rho(\boldsymbol{x}, \boldsymbol{z})$ to be about twice the number of features in the dataset.

Considering $u=\frac{1}{2 d}$, we propose as candidates of $\sigma$ the values $\frac{u}{4}, \frac{u}{2}, u, 2 u, 4 u$. For $d \geq 4$, the range of values $\left[\frac{u}{4}, 4 u\right]$ is enough to cover at least $90 \%$ of the distribution of $\frac{1}{\rho\left(\boldsymbol{x}_{i}, \boldsymbol{x}_{j}\right)}$ when the features are standardized. This means that these proposed parameter candidates ideally iterate over the effective range of candidates of $\sigma$.

To build on the idea of multiple phases again, one could first consider only the candidate $u$, and if needed move to $\frac{u}{2}$ and $2 u$, and then to $\frac{u}{4}$ and $4 u$. For each of these candidates, the best value of $C$ can be selected according to Algorithm 5.1. The best pair $(C, \sigma)$ can be selected according to the best evaluated classification performance.

We know for a fact that the assumption of standardized Normal features is not satisfied in practice, and artificial standardization will not change that. However, it may be the case that the proposed candidates may help to frame the best value of the RBF parameter $\sigma$ when the features are artificially standardized. In Section 5.4, we experimentally verify the usefulness of this proposal.

In Appendix A.1, we show how to carry out the procedure presented in this section using the software package WEKA.

\subsubsection{Non-linear Parameterless Kernels}

The advantages of employing non-linear parameterless kernels are two-fold. First, eliminating one parameter from the selection process allows us to save a lot of calls to the SVM solver. Second, extra parameters may lead to overfitting when the training set size is small and performance evaluation is conducted by cross-validation.

With these motivations in mind, Izmailov et al. (2013) proposed a non-linear parameterless kernel based on the idea of splines, which are piecewise polynomial functions. The authors name their kernel INK-splines, where INK stands for "Infinite Number of Knots". They propose using normalized INK-splines of degree 1 to obtain a one-dimensional kernel which works upon a single feature of the data. The multidimensional kernel is obtained by the multiplication of the one-dimensional kernels for each feature. Reminding the notation $(x \wedge z)=\min (x, z)$, the value of the one-dimensional kernel for feature $j$ of two 
points $\boldsymbol{x}$ and $\boldsymbol{z}$ is calculated as

$$
k_{i n k}\left(x^{j}, z^{j}\right)=1+x^{j} z^{j}+\frac{1}{2}\left|x^{j}-z^{j}\right|\left(x^{j} \wedge z^{j}\right)^{2}+\frac{1}{3}\left(x^{j} \wedge z^{j}\right)^{3},
$$

while the final, multidimensional and normalized, kernel is calculated as

$$
k_{i n k}^{d}(\boldsymbol{x}, \boldsymbol{z})=\prod_{k=1}^{d} \frac{k_{i n k}\left(x^{d}, z^{d}\right)}{\sqrt{k_{i n k}\left(x^{d}, x^{d}\right) k_{i n k}\left(z^{d}, z^{d}\right)}} .
$$

Izmailov et al. (2013) experimentally showed that using INK-splines leads to better or similar classification performance relative to using RBF. Despite these good results, the application of INK-splines is limited to low-dimensional problems. The reason for this is the multiplication used to compute the multidimensional kernel, as numerical difficulties arise with increasing $d$.

Here we focus on another INK-splines kind of kernel that is better suited for general SVM classification than the one proposed by Izmailov et al. (2013). We use the non-linear part of the INK-splines of the zeroeth degree to obtain a one-dimensional kernel for each feature:

$$
k_{\text {min }}\left(x^{j}, z^{j}\right)=\left(x^{j} \wedge z^{j}\right) .
$$

Figure 5.3 depicts the form of $k_{\text {min }}$. The multidimensional kernel is calculated as

$$
k_{\text {min }}^{d}(\boldsymbol{x}, \boldsymbol{z})=\sum_{k=1}^{d} k_{\min }\left(x^{d}, z^{d}\right)
$$

while the corresponding normalized version is

$$
\tilde{k}_{\text {min }}^{d}(\boldsymbol{x}, \boldsymbol{z})=\frac{k_{\text {min }}^{d}(\boldsymbol{x}, \boldsymbol{z})}{\sqrt{k_{\min }^{d}(\boldsymbol{x}, \boldsymbol{x}) k_{\min }^{d}(\boldsymbol{z}, \boldsymbol{z})}} .
$$

Figure 5.4 depicts an example of an SVM classifier obtained using the non-normalized $k_{m i n}^{d}$. We refer to the kernel $k_{m i n}^{d}$ and its normalized version $\tilde{k}_{\min }^{d}$ collectively as the additive min kernel or, for simplicity, the min kernel.

The immediate consequence of using addition instead of multiplication is the ability to use the min kernel in high-dimensional situations. As it can also be used for low-dimensional cases, besides being non-linear and parameterless, this kernel has the potential to be an easy first-choice SVM kernel. This is particularly useful when high or low dimensionality is not clear-cut.

The capacity of the entire set of functions induced by the min kernel is a middle ground between the linear and the INK-splines kernels. Like the linear kernel and unlike INK-splines, the min kernel adds coordinate-wise kernels to obtain a multidimensional kernel. Consequently, there are classifiers which the min (and the linear) kernel cannot 


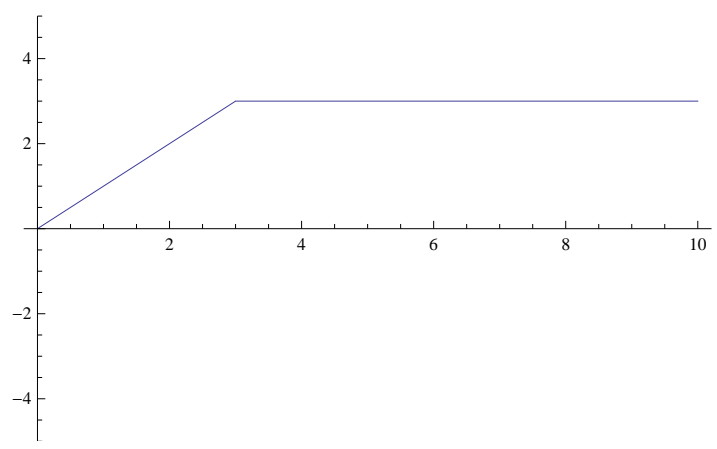

Figure 5.3: Form of the one-dimensional $k_{\min }(x, 3)$ kernel function. Note the hinged (non-linear) pattern of the generated curve.

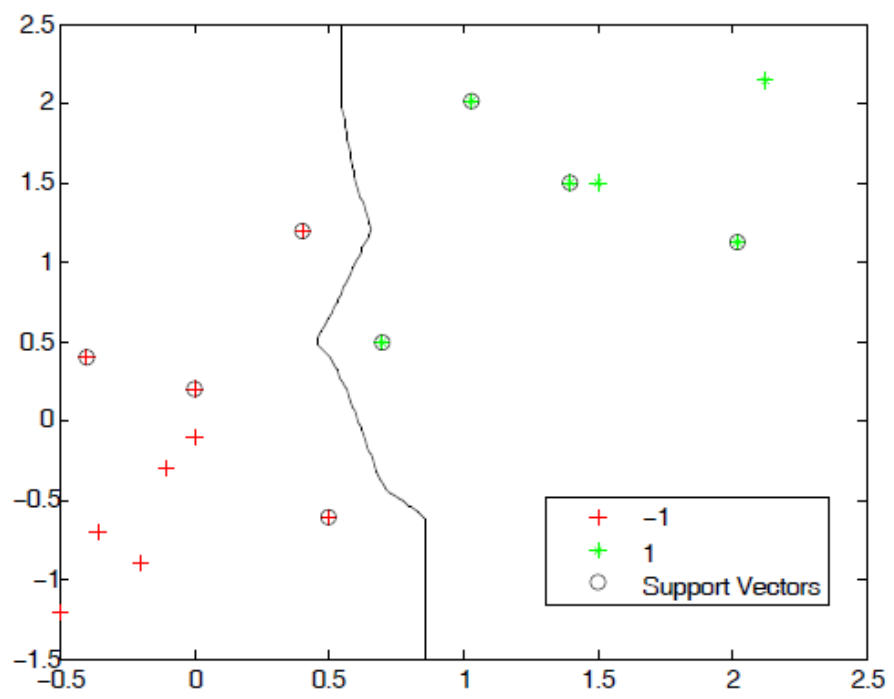

Figure 5.4: SVM classifier obtained using $k_{\min }^{d}$ as the kernel function.

construct. These are typically XOR-like problems - Figure 5.5(b). On the other hand, unlike the linear kernel and like INK-splines, the coordinate-wise min kernel is non-linear, which means that the min kernel (and INK-splines) will be able to handle classification problems like the one in Figure 5.5(a).

\subsection{Experiments}

The experiments in this section use the classification datasets described in Table 5.1. They come from popular online repositories, and were chosen based on whether they provide binary classification tasks with reasonable predictive power. Both challenging and easy classification tasks were considered, ranging from low to high-dimensional, from sparse to dense, and from linearly to non-linearly separable tasks. The datasets span several domains like marketing, physics, medicine, text, genetics, and chemistry.

For evaluation purposes, $30 \%$ of each dataset is randomly held out to form a test set, while the remaining instances form a training set. Both training and test sets retain the original class distribution. This split is performed 30 times for each dataset, each 


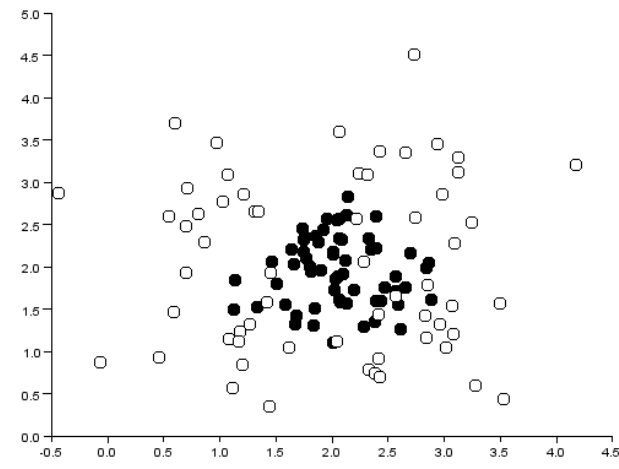

(a)

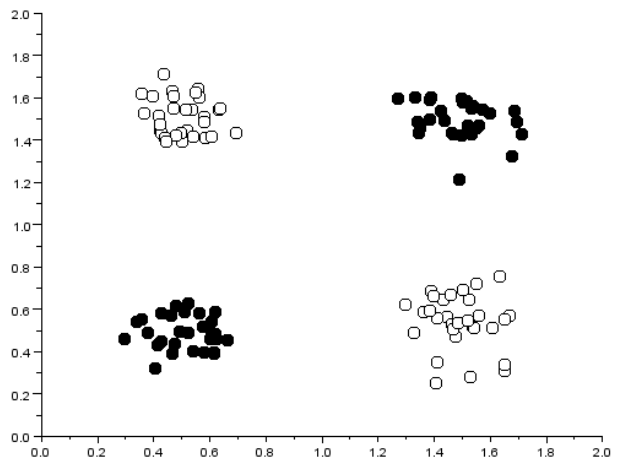

(b)

Figure 5.5: On the left, the binary classification problem is a good match for the additive min kernel. On the right, a classification problem (XOR-like) where the additive min kernel is expected to perform poorly.

time using a different random seed. To enable a stronger comparison, the 30 generated training/test pairs are kept the same throughout the experiments.

Apart from textual datasets, each feature was normalized in the training set to have zero mean and unit variance (standardization). The scale factors obtained for the training set were applied for normalization of the test set. Standardized values greater than 3 or less than -3 were set to 3 and -3 (resp.) in both training and test sets.

For a fixed training/test pair, the training set is used both for training and parameter selection. This is performed on the grounds of the procedure described in Algorithm 5.1 for the selection of $C$ and the procedure described in Section 5.3 for the selection of $\sigma$ and $C$. The best parameter (combination) found by these procedures is then used along with the training set to generate the classifier used for classifying the test set. The function evaluatePerformanceSVM in Algorithm 5.1 is implemented here as a 10-fold cross-validation procedure that returns a cross-validated evaluation performance. The SVM solver we use is the SMO implementation in the WEKA API (version 3.6.10).

We choose the balanced error measure for evaluating classification performance given the occurrence of unbalanced datasets in our experiments. Table 5.2 derives the balanced error from a typical confusion matrix. This measure is used both in the parameter selection and test stages. However, the original SVM formulation does not optimize for the balanced error. To fix this, we have to allow for a different parameter of generalization $C_{i}$ for each point $\left(\boldsymbol{x}_{i}, y_{i}\right)$. Without loss of generality, assume that the negative class $\left(y_{i}=-1\right)$ is the majority class, and let $n_{\ominus}$ and $n_{\oplus}$ be the number of training points within the respective class. In order to approximately minimize the balanced error in SVM, we set $C_{i}=C$ for all points of the majority class and $C_{i}=\frac{n_{\ominus}}{n_{\oplus}} C$ for all points of the minority class. The rationale behind these assignments is to weigh up training errors from the minority class. 


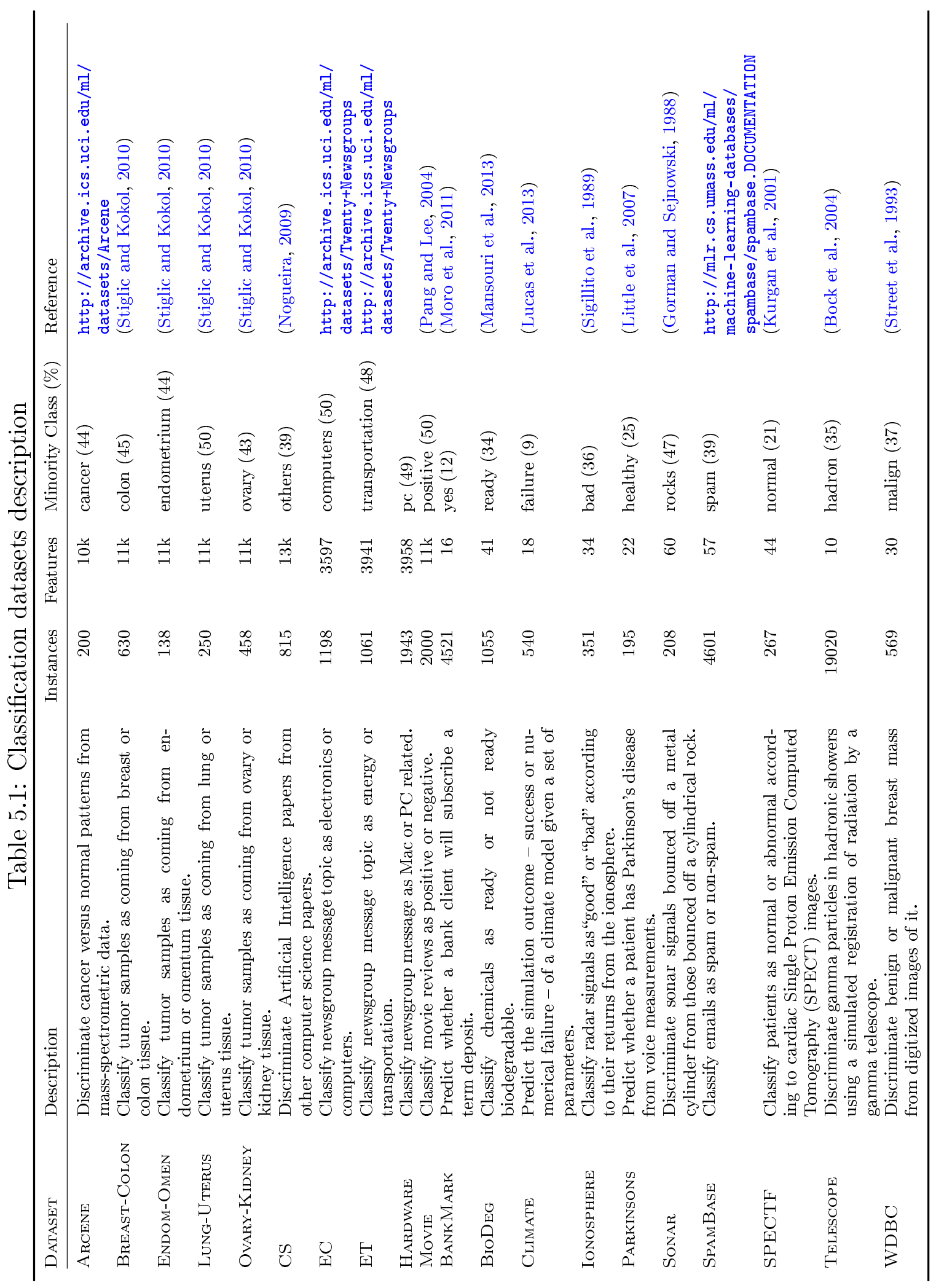


Table 5.2: Balanced error evaluation measure

\begin{tabular}{|c|c|c|c|}
\hline \multicolumn{3}{|r|}{ classified } & \multirow{3}{*}{ Balanced error $=0.5 \times\left(\frac{f p}{f p+t n}+\frac{f n}{f n+t p}\right)$} \\
\hline$\stackrel{0}{ٍ}$ & $\oplus$ & $\begin{array}{ll}\oplus & \ominus \\
t p & f n\end{array}$ & \\
\hline 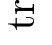 & $\theta$ & $f p \quad t n$ & \\
\hline
\end{tabular}

As to the input parameters of Algorithm 5.1, max_it is set to the minimum of 10 or the number of first phase iterations needed to exceed the upper bound $C_{u p}$. The step size $s$ is set to 10, as a smaller step, say 2, would amount to more SVM calls and probably magnify the cross-validation oscillation issue - Section 5.2.

As kernels, we use $\tilde{k}_{l i n}, k_{r b f}, \tilde{k}_{i n k}^{d}$, and $\tilde{k}_{m i n}^{d}$. Note that RBF does not need be normalized because its values are already in the range $[0,1]$, no matter the chosen value of $\sigma$. Using normalized kernels allows us to use the value of $C_{\text {low }}$ in Expression (5.8). We use:

- the linear kernel for high-dimensional datasets, SONAR, and SPECTF;

- RBF for all datasets except the ones derived from text ${ }^{6}$;

- INK-splines for low-dimensional datasets;

- the min kernel for all datasets.

Next, we analyze the experimental results regarding the proposed procedures for choosing $C$ and $\sigma$, where the concern is to verify the effectiveness of these procedures independent of the kernel function. After that, we focus on the performance of the min kernel. At the end of this section, we explore situations where only 20 or 100 training points are used. In these situations, the experimental protocol is slightly different from the one described so far. We leave the explanation of that protocol to the end of this section.

\subsubsection{Selection of $C$}

We first analyze whether the lower bound for $C$ obtained in Section 5.2 really works as such. By monitoring the evolution of the cross-validation error along with the candidates of $C$, it was possible to ascertain the effectiveness of $C_{\text {low }}$ in Expression (5.8) as a lower bound on $C_{\text {best }}$. According to the results, the average value of $C_{\text {best }}$ tends to be 5 to 6 orders of magnitude greater than $C_{\text {low }}$, which shows that this lower bound is quite loose.

Regarding the cross-validation oscillation issue, the experiments showed that it arises for candidates of $C$ close to $C_{\text {low }}$. This means that the classifier obtained with $C=$ $C_{\text {low }} \times 10$ may get a worse evaluation than the one with $C=C_{\text {low }}$, which constrains the

\footnotetext{
${ }^{6}$ In the case of sparse datasets, standardization is not desirable. Hence, the proposed procedure for $\sigma$ selection is not applicable. Still, the linear kernel is usually preferred over RBF in textual domains.
} 
choice of $C$ to candidates around $C_{\text {low }}$ according to Algorithm 5.1. In these situations, the chosen candidate of $C$ is not expected to provide good performance.

Taking these initial results into account, we also experimented with $C_{l o w}=\frac{1}{2 \sqrt{n}}$. Here we do not consider $C_{\text {low }}=\frac{1}{2 \log n}$ because it was verified from the initial results that such a $C_{\text {low }}$ would be too tight for a couple of datasets. Nevertheless, $C_{l o w}=\frac{1}{2 \log n}$ could be considered when a specific dataset is under investigation.

Tables 5.3 through 5.5 report some of the results of the experiments conducted using $C_{\text {low }}=\frac{1}{2 \sqrt{n}}$. The values shown in these tables are averages over the 30 training/test pairs of each dataset. The columns under "\# SVM Calls" report the number of calls made to the SVM solver, i.e the number of investigated candidates. The columns under "Selected" report the frequency in which $C_{\text {best }}$ : was selected in the second phase (Ph. 2); is less than $C_{\text {low }}\left(<C_{\text {low }}\right)$; and is larger than $C_{u p}\left(>C_{u p}\right)$. The columns under "Balanced Error" report this evaluation measure for the classifier obtained using $C_{\text {best }}$ after the second phase $(\mathrm{S})$, right after the first phase $(\mathrm{F})$, and the difference of the last two.

Note that $C_{\text {low }}=\frac{1}{2 \sqrt{n}}$ also works as a lower bound for $C_{\text {best }}$. Regardless of the kernel function, it contributes to saving 1.5 calls to SVM on average in the first phase. Also, note the small observed difference in the average number of SVM calls for each kernel.

Another advantage over the previous $C_{\text {low }}$ is the decrease in the number of times that the cross-validation oscillation issue appeared. It did happen a couple of times for the ENDOMETRIUM-OMENTUM dataset, which is the smallest dataset in this experimental evaluation. Nevertheless, it is easy to identify such situation by the cross-validation balanced error, which will be close to $50 \%$ in these situations. Another way to prevent it is to make sure that a minimum number of calls is made, say 4, irrespective of the cross-validation error going down.

The results on the last column of Tables 5.3 through 5.5 allow us to answer whether it is worthwhile to conduct the second phase in Algorithm 5.1. With the exception of the linear kernel, which was benefited slightly from the second phase, mainly in the case of the EC and ET datasets, both the INK-splines and min kernels do not seem to benefit from it. The few cases it benefits, such as the EC dataset using the min kernel, are overshadowed by the cases where performing the second phase actually hurts classification performance. This phenomenon is in accordance with the idea that the more candidates evaluated the greater the risk of overfitting in cross-validation, especially for more diverse sets of functions. The linear kernel, being the one which induces the less diverse set of functions, is the only kernel which did not suffer too much from investigating more candidates. This way, we recommend that the second phase in Algorithm 5.1 be skipped. 
Table 5.3: Linear kernel results

\begin{tabular}{|c|c|c|c|c|c|c|c|c|}
\hline \multirow{2}{*}{ Dataset } & \multicolumn{2}{|c|}{ \# SVM Calls } & \multicolumn{3}{|c|}{ Selected $(\%)$} & \multicolumn{3}{|c|}{ Balanced Error (\%) } \\
\hline & Ph. 1 & Ph. 2 & Ph. 2 & $<C_{\text {low }}$ & $>C_{u p}$ & $\mathrm{~S}$ & $\mathrm{~F}$ & $S-F$ \\
\hline ArCEnE & 4.1 & 1.1 & 0.0 & 0.0 & 93.3 & 15.9 & 15.9 & 0.0 \\
\hline Breast-Colon & 5.0 & 1.6 & 16.7 & 0.0 & 40.0 & 3.6 & 3.6 & 0.0 \\
\hline ENDOM-OMEN & 3.8 & 1.1 & 10.0 & 0.0 & 80.0 & 12.0 & 12.0 & -0.1 \\
\hline LUNG-UTERUS & 4.9 & 1.2 & 0.0 & 0.0 & 80.0 & 6.0 & 6.0 & 0.0 \\
\hline OVARY-KIDNEY & 4.3 & 1.5 & 0.0 & 0.0 & 53.3 & 2.2 & 2.2 & 0.0 \\
\hline CS & 3.8 & 1.6 & 40.0 & 0.0 & 33.3 & 3.1 & 3.3 & -0.2 \\
\hline $\mathrm{EC}$ & 3.3 & 1.3 & 96.7 & 0.0 & 0.0 & 24.5 & 25.8 & -1.3 \\
\hline $\mathrm{ET}$ & 3.5 & 1.5 & 96.7 & 0.0 & 0.0 & 8.0 & 9.3 & -1.2 \\
\hline HARDWARE & 4.1 & 1.8 & 23.3 & 0.0 & 20.0 & 7.1 & 7.1 & 0.0 \\
\hline Movie & 4.0 & 2.0 & 0.0 & 0.0 & 0.0 & 18.2 & 18.2 & 0.0 \\
\hline SONAR & 4.5 & 1.8 & 26.7 & 0.0 & 10.0 & 31.4 & 31.6 & -0.2 \\
\hline SPECTF & 4.5 & 1.8 & 33.3 & 0.0 & 0.0 & 31.5 & 31.2 & 0.4 \\
\hline Avg. & 4.1 & 1.5 & 28.6 & 0.0 & 34.2 & 13.6 & 13.8 & -0.2 \\
\hline
\end{tabular}

Table 5.4: INK-splines kernel results

\begin{tabular}{|c|c|c|c|c|c|c|c|c|}
\hline \multirow{2}{*}{ Dataset } & \multicolumn{2}{|c|}{ \# SVM Calls } & \multicolumn{3}{|c|}{ Selected $(\%)$} & \multicolumn{3}{|c|}{ Balanced Error (\%) } \\
\hline & Ph. 1 & Ph. 2 & Ph. 2 & $<C_{\text {low }}$ & $>C_{u p}$ & $\mathrm{~S}$ & $\mathrm{~F}$ & $S-F$ \\
\hline BANKMARK & 3.5 & 1.7 & 60.0 & 0.0 & 0.0 & 17.9 & 18.0 & -0.1 \\
\hline BIODEG & 4.6 & 1.8 & 46.7 & 0.0 & 0.0 & 13.7 & 13.8 & -0.1 \\
\hline Climate & 3.0 & 2.0 & 23.3 & 0.0 & 0.0 & 13.2 & 12.6 & 0.6 \\
\hline IONOSPHERE & 4.0 & 1.7 & 16.7 & 0.0 & 16.7 & 7.8 & 8.0 & -0.2 \\
\hline PARKINSONS & 3.6 & 1.2 & 33.3 & 0.0 & 76.7 & 10.5 & 9.9 & 0.5 \\
\hline SONAR & 3.0 & 1.0 & 16.7 & 0.0 & 100.0 & 14.1 & 13.8 & 0.3 \\
\hline SPAMBASE & 5.0 & 1.7 & 36.7 & 0.0 & 0.0 & 5.6 & 5.6 & 0.0 \\
\hline SPECTF & 3.0 & 1.8 & 30.0 & 0.0 & 10.0 & 22.8 & 22.7 & 0.1 \\
\hline Telescope & 5.8 & 1.8 & 53.3 & 0.0 & 0.0 & 15.0 & 15.0 & 0.0 \\
\hline WDBC & 4.0 & 1.8 & 13.3 & 0.0 & 10.0 & 2.7 & 2.8 & -0.2 \\
\hline Avg. & 4.0 & 1.7 & 33.0 & 0.0 & 21.3 & 12.3 & 12.2 & 0.1 \\
\hline
\end{tabular}

Table 5.5: Min kernel results

\begin{tabular}{|c|c|c|c|c|c|c|c|c|}
\hline \multirow{2}{*}{ DAtaset } & \multicolumn{2}{|c|}{ \# SVM Calls } & \multicolumn{3}{|c|}{ Selected $(\%)$} & \multicolumn{3}{|c|}{ Balanced Error (\%) } \\
\hline & Ph. 1 & Ph. 2 & Ph. 2 & $<C_{\text {low }}$ & $>C_{u p}$ & $\mathrm{~S}$ & $\mathrm{~F}$ & $S-F$ \\
\hline ArCEnE & 4.0 & 1.0 & 13.3 & 0.0 & 100.0 & 14.7 & 14.4 & 0.3 \\
\hline Breast-Colon & 4.0 & 1.0 & 6.7 & 0.0 & 100.0 & 3.6 & 3.5 & 0.0 \\
\hline ENDOM-OMEN & 3.5 & 1.2 & 3.3 & 0.0 & 73.3 & 17.1 & 17.1 & 0.0 \\
\hline LUNG-UTERUS & 4.0 & 1.3 & 10.0 & 0.0 & 70.0 & 6.6 & 6.6 & 0.0 \\
\hline Ovary-Kidney & 3.9 & 1.3 & 0.0 & 0.0 & 66.7 & 2.4 & 2.4 & 0.0 \\
\hline $\mathrm{CS}$ & 3.0 & 1.0 & 20.0 & 0.0 & 100.0 & 1.4 & 1.4 & 0.0 \\
\hline $\mathrm{EC}$ & 4.0 & 2.0 & 83.3 & 0.0 & 0.0 & 17.5 & 18.9 & -1.4 \\
\hline $\mathrm{ET}$ & 4.0 & 2.0 & 20.0 & 0.0 & 0.0 & 5.8 & 5.6 & 0.1 \\
\hline HARDWARE & 4.0 & 1.6 & 3.3 & 0.0 & 40.0 & 6.8 & 6.8 & 0.0 \\
\hline Movie & 4.0 & 1.9 & 16.7 & 0.0 & 13.3 & 16.3 & 16.3 & 0.0 \\
\hline BANKMARK & 5.2 & 1.7 & 53.3 & 0.0 & 0.0 & 17.7 & 17.8 & -0.1 \\
\hline BIODEG & 4.9 & 1.9 & 30.0 & 0.0 & 0.0 & 14.4 & 14.2 & 0.2 \\
\hline Climate & 3.0 & 1.6 & 36.7 & 0.0 & 3.3 & 12.7 & 12.4 & 0.2 \\
\hline IONOSPHERE & 4.2 & 1.7 & 13.3 & 0.0 & 26.7 & 9.5 & 9.5 & 0.0 \\
\hline PARKINSONS & 4.0 & 1.0 & 40.0 & 0.0 & 93.3 & 9.1 & 9.3 & -0.3 \\
\hline Sonar & 4.0 & 1.0 & 13.3 & 0.0 & 96.7 & 15.8 & 15.6 & 0.2 \\
\hline SpamBase & 5.9 & 1.9 & 46.7 & 0.0 & 0.0 & 5.7 & 5.7 & 0.0 \\
\hline SPECTF & 3.9 & 1.9 & 56.7 & 0.0 & 3.3 & 23.1 & 23.6 & -0.5 \\
\hline Telescope & 5.0 & 1.9 & 30.0 & 0.0 & 0.0 & 17.1 & 17.1 & 0.0 \\
\hline WDBC & 4.5 & 1.4 & 26.7 & 0.0 & 43.3 & 2.9 & 2.9 & 0.0 \\
\hline Avg. & 4.2 & 1.5 & 26.2 & 0.0 & 41.5 & 11.0 & 11.1 & -0.1 \\
\hline
\end{tabular}




\subsubsection{Selection of $\sigma$}

Here we investigate whether the proposed candidates of $\sigma$ - Section 5.3.1 - provide a good frame for the best value of $\sigma$. For that, the squared distance $\rho\left(\boldsymbol{x}_{i}, \boldsymbol{x}_{j}\right)=\left\|\boldsymbol{x}_{i}-\boldsymbol{x}_{j}\right\|_{2}^{2}$ of all pairs of training points were calculated. Table 5.6 reports the average over the 30 trials of the 5- and 95-percentiles of the distribution of squared distances. Along with the percentiles, the table shows the endpoint candidates calculated as described in Section 5.3.1. Ideally, the smaller candidate should be close to or less than the 5-percentile, whereas the larger candidate should be close to or larger than the 95-percentile. The columns under "Average" report the calculated average of $\rho\left(\boldsymbol{x}_{i}, \boldsymbol{x}_{j}\right)$ and the average as given by $2 d$.

Note that the largest candidate was always large than the 95-percentile. For the smallest candidate, though, it was sometimes larger than the 5-percentile, yet not much larger - cf. Biodeg, Ionosphere, Parkinsons, SpamBase, SPECTF, Telescope, and WDBC. As to the averages, note that the approximation tends to be quite good in some cases - cf. Climate, Ionosphere, and Telescope - and in the same order of magnitude for most cases. Only for the SPAMBASE dataset have the approximations gone a little awry, though they cover a good portion of the distribution of $\rho\left(\boldsymbol{x}_{i}, \boldsymbol{x}_{j}\right)$ for this dataset.

In order to evaluate the performance of the classifiers obtained through the procedure for selecting $\sigma$, we set $C_{\text {low }}=\frac{1}{2 \sqrt{n}}$. As the $\sigma$ selection procedure is divided into three phases, Table 5.7 reports the classification performance considering the best parameter combination in each phase. The column under $\sigma_{\min }\left(\sigma_{\max }\right)$ reports the frequency with which the smallest (largest) candidate of $\sigma$ was selected.

Note that, on average, there is a balance in the frequency with which the endpoint candidates are selected $-17.1 \%$ for $\sigma_{\min }$ and $20.9 \%$ for $\sigma_{\max }$. Regarding classification performance, it is important to observe that the performance gains brought by considering $\sigma$ candidates in phases 2 and 3 tend to occur consistently. In other words, it was worthwhile to consider these candidates.

Another way of verifying whether we are considering a good range of $\sigma$ candidates is to compare the performance of the classifiers with the RBF kernel to the other kernels. This way, Figures 5.6(a) and 5.6(b) show scatter plots of the balanced error for each training/test pair of each dataset. Note that the INK-splines results are compatible to those of RBF, whereas the linear kernel has an advantage over RBF in high-dimensional datasets, which is in accordance with previous observations (Hsu et al., 2003). It is worth observing that the few circles indicating a bad performance of the linear kernel correspond to the cases where the cross-validation oscillation problem occurred. 
Table 5.6: RBF inner squared distance distribution

\begin{tabular}{|c|c|c|c|c|c|c|}
\hline \multirow{2}{*}{ Dataset } & \multicolumn{2}{|c|}{$\underline{\left\|\boldsymbol{x}_{i}-\boldsymbol{x}_{j}\right\|^{2} \text { Percentiles }}$} & \multicolumn{2}{|c|}{ Endpoint Candidates } & \multicolumn{2}{|c|}{ Average } \\
\hline & 5 & 95 & Smaller & Larger & Sample & Formula \\
\hline Arcene & $6.5 \mathrm{E}+3$ & $2.6 \mathrm{E}+4$ & $5.0 \mathrm{E}+3$ & $8.0 \mathrm{E}+4$ & $1.6 \mathrm{E}+4$ & $2.0 \mathrm{E}+4$ \\
\hline Breast-Colon & $9.2 \mathrm{E}+3$ & $2.5 \mathrm{E}+4$ & $5.5 \mathrm{E}+3$ & $8.7 \mathrm{E}+4$ & $1.7 \mathrm{E}+4$ & $2.2 \mathrm{E}+4$ \\
\hline ENDOM-OMEN & $1.0 \mathrm{E}+4$ & $2.6 \mathrm{E}+4$ & $5.5 \mathrm{E}+3$ & $8.7 \mathrm{E}+4$ & $1.7 \mathrm{E}+4$ & $2.2 \mathrm{E}+4$ \\
\hline Lung-Uterus & $9.6 \mathrm{E}+3$ & $2.5 \mathrm{E}+4$ & $5.5 \mathrm{E}+3$ & $8.7 \mathrm{E}+4$ & $1.7 \mathrm{E}+4$ & $2.2 \mathrm{E}+4$ \\
\hline OVARY-KIDNEy & $7.1 \mathrm{E}+3$ & $2.5 \mathrm{E}+4$ & $5.5 \mathrm{E}+3$ & $8.7 \mathrm{E}+4$ & $1.6 \mathrm{E}+4$ & $2.2 \mathrm{E}+4$ \\
\hline BANKMARK & 32.1 & 100.1 & 24 & 384 & 65.7 & 96 \\
\hline BIODEG & 12.3 & 124.4 & 20.5 & 328 & 54.7 & 82 \\
\hline Climate & 20.9 & 53.1 & 9 & 144 & 36.0 & 36 \\
\hline IONOSPHERE & 4.8 & 149.4 & 17 & 272 & 66.0 & 68 \\
\hline PARKINSONS & 6.1 & 119.5 & 11 & 176 & 37.5 & 44 \\
\hline SONAR & 38.7 & 217.7 & 30 & 480 & 112.9 & 120 \\
\hline SpamBase & 12.1 & 84.3 & 28.5 & 456 & 41.1 & 114 \\
\hline SPECTF & 18.5 & 220.4 & 22 & 352 & 76.4 & 88 \\
\hline Telescope & 2.4 & 52.7 & 5 & 80 & 17.9 & 20 \\
\hline WDBC & 9.1 & 140.6 & 15 & 240 & 52.9 & 60 \\
\hline
\end{tabular}

Table 5.7: RBF kernel results

\begin{tabular}{|c|c|c|c|c|c|c|c|c|c|c|c|}
\hline \multirow{2}{*}{ DATASET } & \multirow{2}{*}{ \# SVM Calls } & \multicolumn{4}{|c|}{ Selected $(\%)$} & \multicolumn{6}{|c|}{ Balanced Error (\%) } \\
\hline & & Ph. 2 & Ph. 3 & $\sigma_{\min }$ & $\sigma_{\max }$ & $\mathrm{T}$ & $\mathrm{S}$ & $\mathrm{F}$ & $\mathrm{T}-\mathrm{S}$ & $\mathrm{T}-\mathrm{F}$ & $S-F$ \\
\hline Arcene & 22.8 & 53.3 & 16.7 & 16.7 & 0.0 & 14.1 & 14.1 & 14.4 & 0.0 & -0.3 & -0.3 \\
\hline Breast-Colon & 26.9 & 23.3 & 40.0 & 40.0 & 0.0 & 3.7 & 3.8 & 3.9 & -0.1 & -0.2 & -0.1 \\
\hline ENDOM-OMEN & 21.5 & 36.7 & 13.3 & 10.0 & 3.3 & 7.2 & 7.2 & 7.5 & 0.0 & -0.3 & -0.3 \\
\hline LuNG-UTERUS & 23.6 & 36.7 & 36.7 & 36.7 & 0.0 & 6.8 & 7.0 & 7.0 & -0.2 & -0.2 & 0.0 \\
\hline OVARY-KIDNEY & 24.1 & 23.3 & 13.3 & 13.3 & 0.0 & 2.3 & 2.3 & 2.3 & -0.1 & -0.1 & 0.0 \\
\hline BANKMARK & 26.9 & 56.7 & 26.7 & 23.3 & 3.3 & 18.2 & 18.2 & 18.1 & 0.1 & 0.2 & 0.1 \\
\hline BioDeg & 32.8 & 36.7 & 63.3 & 0.0 & 63.3 & 12.9 & 13.1 & 13.6 & -0.2 & -0.7 & -0.5 \\
\hline Climate & 22.9 & 36.7 & 40.0 & 40.0 & 0.0 & 12.3 & 12.7 & 12.0 & -0.3 & 0.3 & 0.7 \\
\hline IONOSPHERE & 30.4 & 13.3 & 70.0 & 0.0 & 70.0 & 7.0 & 7.7 & 7.7 & -0.7 & -0.7 & 0.0 \\
\hline PARKINSONS & 29.3 & 20.0 & 63.3 & 6.7 & 56.7 & 9.4 & 9.8 & 11.7 & -0.5 & -2.3 & -1.9 \\
\hline SONAR & 26.1 & 53.3 & 36.7 & 0.0 & 36.7 & 14.4 & 14.8 & 17.1 & -0.4 & -2.6 & -2.2 \\
\hline SpamBase & 37.0 & 20.0 & 60.0 & 33.3 & 26.7 & 6.3 & 6.3 & 6.4 & 0.0 & 0.0 & 0.0 \\
\hline SPECTF & 26.0 & 46.7 & 50.0 & 20.0 & 30.0 & 23.5 & 23.3 & 24.2 & 0.2 & -0.8 & -0.9 \\
\hline Telescope & 23.1 & 26.7 & 23.3 & 10.0 & 13.3 & 38.6 & 39.3 & 39.3 & -0.8 & -0.7 & 0.0 \\
\hline WDBC & 30.7 & 63.3 & 16.7 & 6.7 & 10.0 & 2.7 & 2.7 & 2.6 & 0.0 & 0.2 & 0.2 \\
\hline Avg. & 26.9 & 36.4 & 38.0 & 17.1 & 20.9 & 12.0 & 12.2 & 12.5 & -0.2 & -0.6 & -0.4 \\
\hline
\end{tabular}

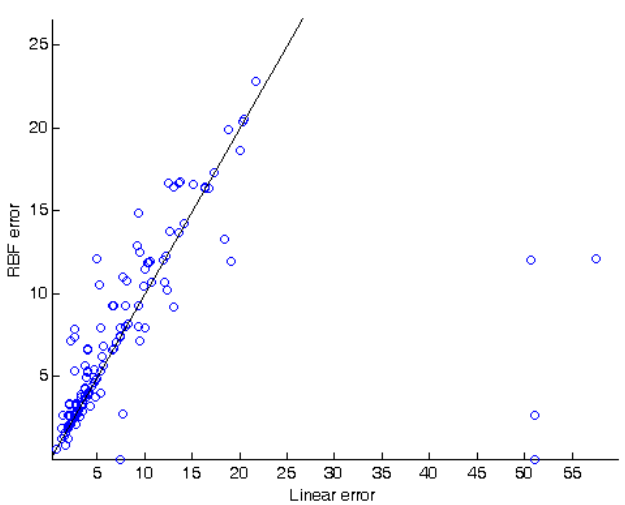

(a) Linear versus $\mathrm{RBF}$

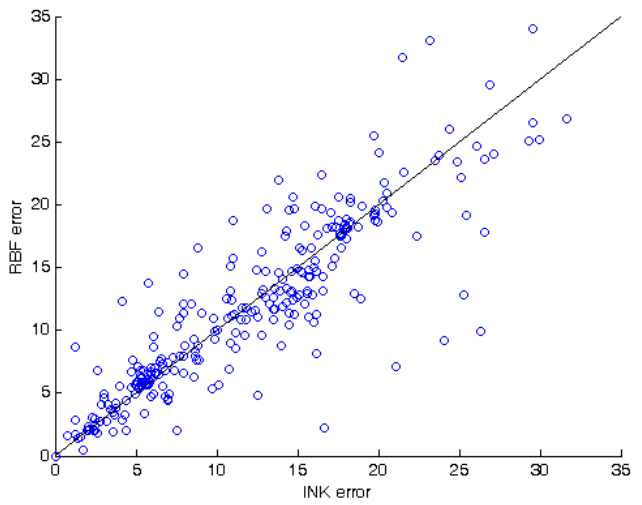

(b) INK-splines versus RBF

Figure 5.6: Scatter plots of the balanced error 


\subsubsection{Performance of the Min Kernel}

Here we compare the min kernel performance to the other kernels involved in this experimental evaluation. Once again, $C_{\text {low }}=\frac{1}{2 \sqrt{n}}$. Figures 5.7 through 5.9 show scatter plots of the balanced error for each training/test pair of each dataset. A comparison of the average balanced error per dataset is provided in Tables 5.8 through 5.10 in the columns under " $n=$ training".

For the non-textual high-dimensional datasets in Table 5.8, most of the results coming from the linear and min kernels are compatible. The exception is again due to the crossvalidation oscillation problem in the ENDOMETRIUM-OMENTUM dataset, which penalized the min more than the linear kernel. The textual datasets, however, clearly favored the min kernel - Table 5.9. Moreover, as it can be verified from the balanced error entries of SONAR and SPECTF in Tables 5.3 and 5.5, the min kernel performs much better than the linear one when dealing with low-dimensional datasets in which the training points can be linearly separable.

The comparison between the min kernel and INK-splines is confined to the lowdimensional datasets, as the INK-splines kernel does not work with high-dimensional problems at all. For RBF, all datasets but the textual ones are considered in this comparison. The average results show an advantage of the INK-splines and RBF kernels for some datasets - cf. IONOSPHERE and SONAR - , though in some others, the min kernel is slightly favored - cf. BANKMARK and PARKINSONS. According to the scatter plots, there is a tendency for better INK-splines and RBF classifiers in low-dimensional datasets. That is understandable because, as explained in Section 5.3.2, the min kernel has less capacity than INK-splines and RBF kernels.

Finally, we analyze the effect of the training set size on the relative performance of the investigated kernel functions. For that, each of the original 30 training sets of each dataset were randomly reduced to contain 20 or 100 points, once again retaining the original class distribution. The test sets were kept intact. As the training sets have a small number of points, and to avoid the cross-validation oscillation problem, we do not stop the first phase in Algorithm 5.1 when the cross-validation error goes up. Instead, the first phase runs until $C_{\text {curr }}>C_{u p}$.

Tables 5.8 through 5.10 report the balanced error results. Overall, the effect of decreasing the training set size is to increase the differences observed when using the entire training set. It was not clear whether cross-validation for small training set sizes was negatively affected by the number of parameters and the capacity of the set of functions, contrary to what is claimed in (Izmailov et al., 2013). 


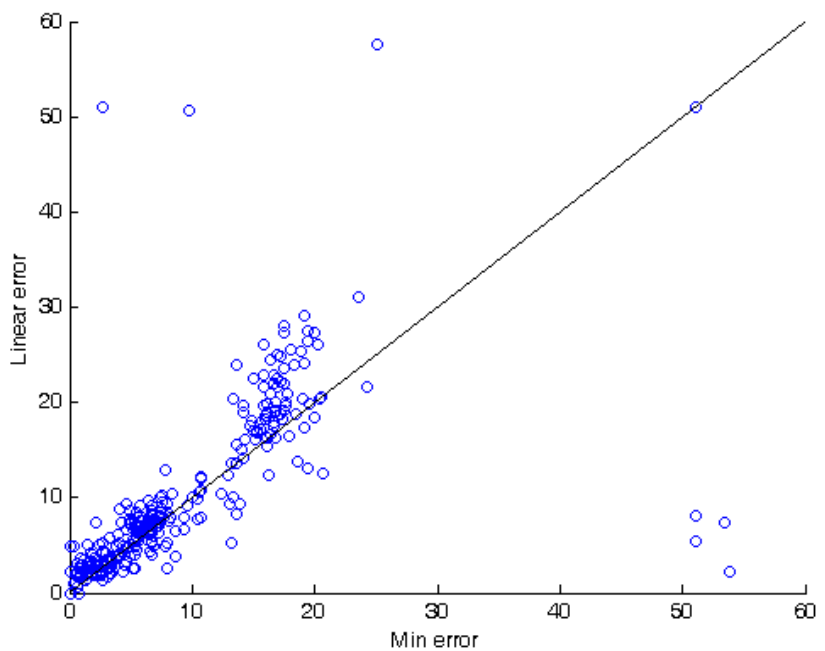

Figure 5.7: Min kernel versus Linear

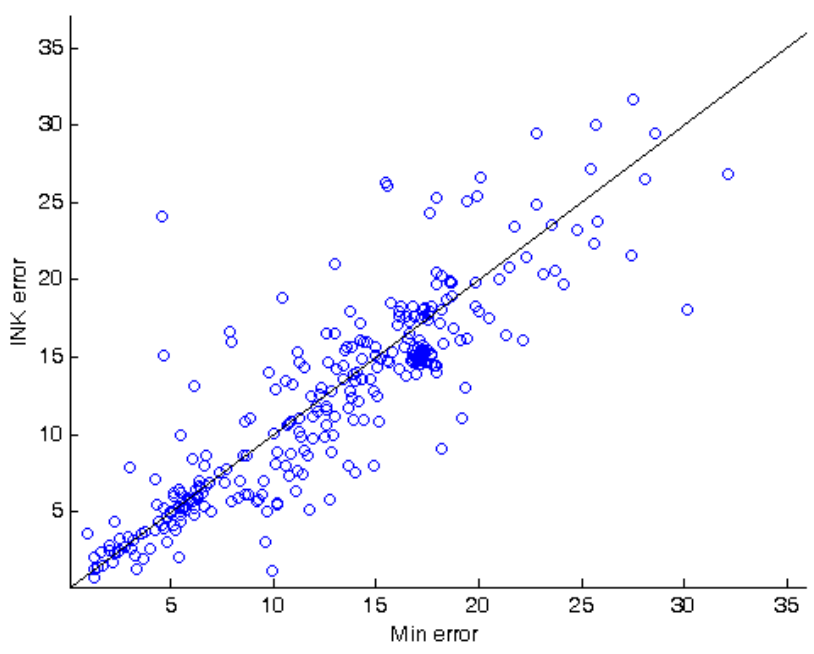

Figure 5.8: Min kernel versus INK-splines

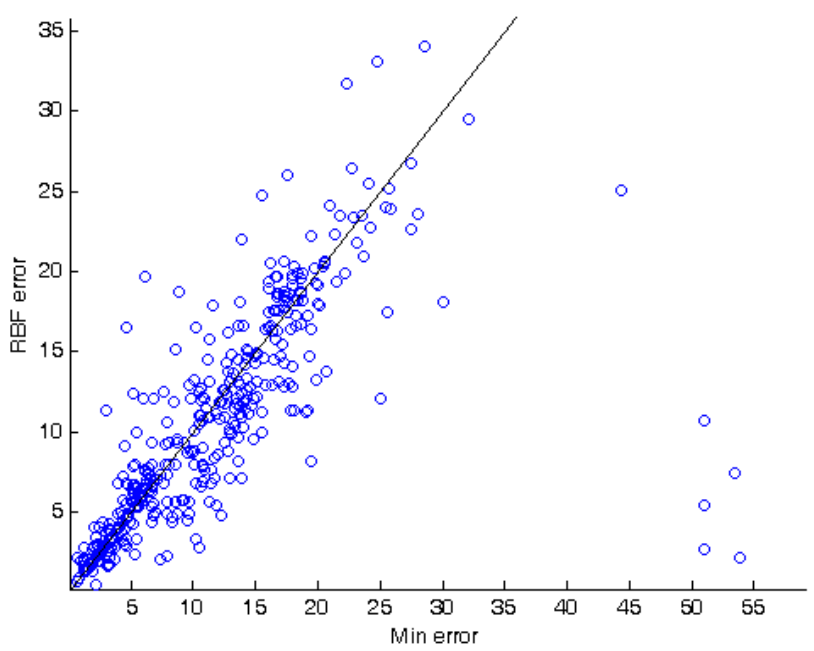

Figure 5.9: Min kernel versus RBF 

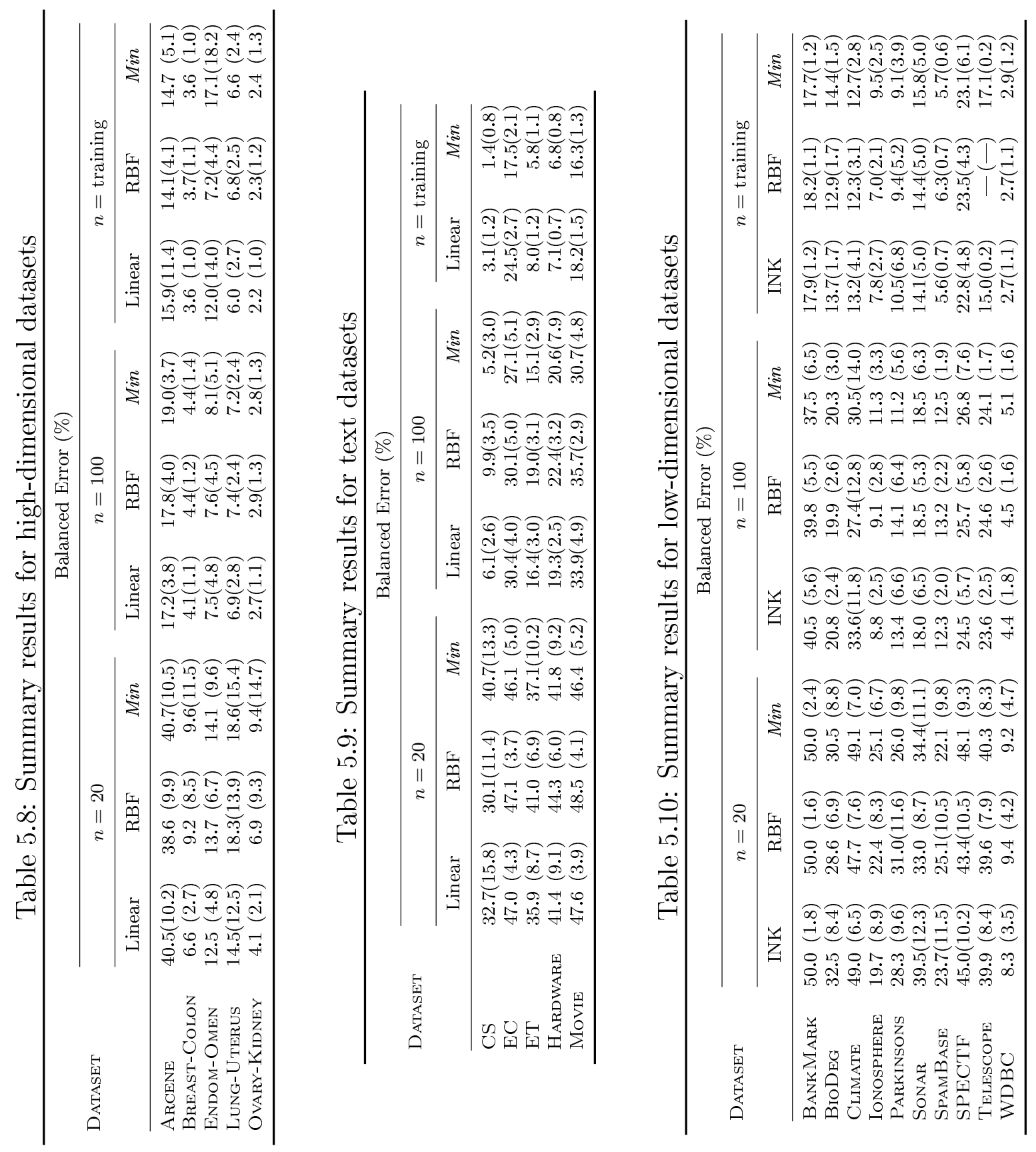


\subsection{Final Remarks}

To the best of our knowledge, the subject of obtaining a not-too-loose lower bound $C_{\text {low }}$ for the best parameter of generalization $C_{\text {best }}$ has not been explored in the literature. In this chapter, we obtained such a lower bound when the kernel function is normalized $\left(C_{l o w}=\frac{1}{2 n}\right)$, and were able to propose an improved one $\left(C_{l o w}=\frac{1}{2 \sqrt{n}}\right)$. We tested this improved lower bound experimentally, as demonstrating its effectiveness theoretically without further assumptions seems a difficult task.

Regarding the proposed procedure for the selection of $\sigma$ in RBF, its importance goes beyond the reduction in the number of SVM calls. It may also be responsible for alleviating the problems imputed to RBF when using cross-validation for parameter selection (Izmailov et al., 2013). As fewer candidates of $\sigma$ tend to be investigated, the RBF kernel provided classifiers that followed the performance of parameterless kernels in small training sets. In any case, using the RBF kernel continues to be more computationally expensive than the INK-splines and min kernels.

As to the additive min kernel, we reinforce that it was suggested in this chapter as a follow-up of Izmailov et al. (2013) work, which emphasized the importance of non-linear parameterless kernels and pointed to the class of INK-splines as important candidates for implementing such kernels. Still, a search through the literature revealed that this kernel was proposed before in the computer vision field under the name "histogram intersection kernel" (Maji et al., 2013). We also found that the idea of using hinged hyperplanes as decision functions goes back to Breiman (1993) work, although it was unrelated to SVM.

Despite previous work, the min kernel has gone practically unnoticed over the years. In this work we show that it has the caliber to be a useful kernel in a variety of domains, mainly the textual one. We recommend its use not only for its good performance, but also because it is a non-linear parameterless kernel that can be applied to both low and highdimensional problems. These desirable traits, which are not available in currently popular kernels, provides for an economic parameter selection procedure in SVM classification. The main problem with the min kernel is its capacity limitation, as it was pointed out in Section 5.3.2. However, this limitation did not prevent it from obtaining better or comparable results in most of the datasets we experimented with. Altogether, these arguments put the additive min kernel in a position to be a first-choice kernel for economic SVM classification. 


\section{Chapter 6}

\section{Statistical and Heuristic Parameter Selection in RLS}

Non-linear regression estimation is an important modeling tool throughout the sciences. Several methods have been proposed to tackle this estimation problem, with the most flexible and powerful ones falling into the category of kernel methods (Schölkopf and Smola, 2002). Among these is the Regularized Least Squares method (Wahba, 1990; Girosi, 1997; Rifkin, 2006), which enjoys good statistical and computational properties.

The RLS method bears some resemblance with Support Vector Machines (Cortes and Vapnik, 1995). It also tries to minimize the training error subject to constraints on the diversity of the set of admissible functions. Thus, it is not surprising that RLS has a parameter of regularization $\gamma$ that must be properly selected in order to control the tradeoff between training error and diversity of the set of functions. As RLS is a kernel method, the selection of a kernel function $k$ is also essential.

The choice of the regularization parameter in the density ratio algorithm DRE-V Section 3.4.2 - can be regarded as the problem of selecting the homonymous parameter in RLS. After an initial investigation in the realm of DRE-V, we expanded this research topic to the context of the RLS method.

The particular characteristics of RLS allow for a rich set of potential parameter selection procedures. Most of the time, however, only cross-validation is considered, usually along with Gaussian RBF kernels. In this chapter, we follow two lines of investigation that may enable faster and/or more accurate RLS parameter selection. First, we consider alternative, statistical or heuristic, parameter selection methods that have been shown to perform well for other regression methods and, to the best of our knowledge, have remained unexplored in the context of RLS. Then, we consider once again the idea of constructing parameterless non-linear kernels by summing one-dimensional spline kernels.

This chapter is organized as follows. Section 6.1 provides an overview of RLS and the challenges that arise in common procedures of RLS parameter selection. Sections 6.2 and 
6.3 describe statistical and heuristic procedures used in this work to perform parameter selection. Section 6.4 describes the issues surrounding the choice of a kernel function and presents formal arguments in defense of the additive INK-splines kernel. Section 6.5 reports experimental evidence in favor of the additive INK-splines kernel and also the results of the experimental evaluation of the considered parameter selection procedures.

\subsection{Overview of RLS}

In a nutshell, the regularized least squares method works as follows. Using a sequence of training data

$$
\left(\boldsymbol{x}_{1}, y_{1}\right), \ldots,\left(\boldsymbol{x}_{n}, y_{n}\right), \quad \boldsymbol{x} \in \boldsymbol{R}^{d}, y \in \boldsymbol{R}
$$

drawn i.i.d from a fixed but unknown probability distribution $P(\boldsymbol{x}, y)$, a function $f_{k, \gamma}$ is obtained as the solution of the minimization problem

$$
f_{k, \gamma}=\underset{f \in \mathcal{F}_{K}}{\arg \min }\left[\frac{1}{n} \sum_{i=1}^{n}\left(y_{i}-f\left(\boldsymbol{x}_{i}\right)\right)^{2}+\gamma\|f\|_{K}^{2}\right],
$$

where $\gamma>0$ is a real-valued regularization parameter and $\mathcal{F}_{K}$ is a Reproducing Kernel Hilbert Space (RKHS) induced by a kernel function $k:\left(\boldsymbol{R}^{d} \times \boldsymbol{R}^{d}\right) \mapsto \boldsymbol{R}$. A function $f \in \mathcal{F}_{K}$ with bounded $\|f\|_{K}$ satisfies some regularity properties, hence the use of the term "regularized" to name the method.

In order to apply RLS successfully, that is, to use $f_{k, \gamma}(\boldsymbol{x})$ to predict the output $y$ of unseen $\boldsymbol{x}$, we must find a $f_{k, \gamma}$ that 1 ) fits the training data well (i.e minimizes the squared loss) and 2) is a reasonably smooth function (i.e minimizes the norm $\|\cdot\|_{K}$ ). As one can always minimize one of these factors at the expense of the other, proper selection of both the kernel function $k$ and regularization parameter $\gamma$ is indispensable for the generalization performance of RLS.

\subsubsection{Solving the RLS Minimization Problem}

Here we assume that the kernel function $k$ has already been chosen, including its eventual parameters. By the representer theorem (Kimeldorf and Wahba, 1970), the minimizer in Expression (6.2) has an expansion of the form:

$$
f(\boldsymbol{x})=\sum_{i=1}^{n} \alpha_{i} k\left(\boldsymbol{x}_{i}, \boldsymbol{x}\right), \quad \alpha_{i} \in R
$$

Hereafter, we denote by $\boldsymbol{y}$ the $n \times 1$ vector $\left[y_{1}, \ldots, y_{n}\right]^{\top}$ and by $K$ the $n \times n$ matrix with entries $k_{i j}=k\left(\boldsymbol{x}_{i}, \boldsymbol{x}_{j}\right)$. We also denote by $\boldsymbol{\alpha}$ the $n \times 1$ vector $\left[\alpha_{1}, \ldots, \alpha_{n}\right]^{\top}$. 
Plugging Expression (6.3) into Expression (6.2) yields the following expression for calculating the squared loss:

$$
\frac{1}{n} \sum_{i=1}^{n}\left(y_{i}-f\left(\boldsymbol{x}_{i}\right)\right)^{2}=\frac{1}{n} \boldsymbol{\alpha}^{\top} K K \boldsymbol{\alpha}-\frac{2}{n} \boldsymbol{\alpha}^{\top} K \boldsymbol{y}+\text { const } .
$$

Moreover, by considering the definition of the norm in an RKHS, we have that $\|f\|_{K}=$ $\boldsymbol{\alpha}^{\top} K \boldsymbol{\alpha}$. Ignoring the constant term in Expression (6.4), we arrive at the following quadratic minimization problem for Expression (6.2):

$$
\boldsymbol{\alpha}_{\gamma}=\underset{\boldsymbol{\alpha} \in R^{n}}{\arg \min }\left[\frac{1}{n} \boldsymbol{\alpha}^{\top} K K \boldsymbol{\alpha}-\frac{2}{n} \boldsymbol{\alpha}^{\top} K \boldsymbol{y}+\gamma \boldsymbol{\alpha}^{\top} K \boldsymbol{\alpha}\right] .
$$

A necessary and sufficient condition for the solution of this minimization problem is obtained by taking the derivative of the functional in Expression (6.5) with respect to each $\alpha_{i}$ and equating it to zero. By doing this, we arrive at the following system of linear equations:

$$
\frac{2}{n} K K \boldsymbol{\alpha}_{\gamma}-\frac{2}{n} K \boldsymbol{y}+2 \gamma K \boldsymbol{\alpha}_{\gamma}=0
$$

Denoting by $I$ the $n \times n$ identity matrix, extracting $\frac{1}{n}$ from $\gamma$, and solving for $\boldsymbol{\alpha}_{\gamma}$ in Expression (6.6), we arrive at the solution of the minimization problem in Expression (6.5):

$$
\boldsymbol{\alpha}_{\gamma}=(K+\gamma I)^{-1} \boldsymbol{y}
$$

Plugging Expression (6.7) into Expression (6.3) yields the closed-form expression for the minimizer in Expression (6.2).

Most parameter selection procedures require the calculation of $\boldsymbol{\alpha}_{\gamma}$ for a fair number of $\gamma$ candidates. In order to avoid solving one system of linear equations for each new $\gamma$, one can take advantage of the eigendecomposition of the kernel matrix: $K=U \Lambda U^{\top}$, where $U$ is the $n \times n$ matrix formed by the eigenvectors of $K$ and $\Lambda$ is the $n \times n$ diagonal matrix containing the eigenvalues $\lambda_{1}>\lambda_{2}>\ldots>\lambda_{n}$ of $K$. Denoting by $\Lambda_{\gamma}$ the $n \times n$ diagonal matrix with entries $\lambda_{i i}=\frac{1}{\lambda_{i}+\gamma}, \boldsymbol{\alpha}_{\gamma}$ can be calculated by performing matrix multiplications:

$$
\boldsymbol{\alpha}_{\gamma}=U \Lambda_{\gamma} U^{\top} \boldsymbol{y}
$$

Both the eigendecomposition of a matrix and a typical algorithm for solving a dense system of linear equations can be carried out in $O\left(n^{3}\right)$ time, with smaller constants for the latter. However, the eigendecomposition may still be preferable depending on $n$ and the number of $\gamma$ candidates considered.

Expression (6.8) contributes to establish a frame for effective values of $\gamma$ candidates. Note that

$$
\boldsymbol{\alpha}_{\gamma} \approx \begin{cases}\frac{1}{\gamma} \boldsymbol{y}, & \gamma \gg \lambda_{1} \\ K \boldsymbol{y}, & \gamma \ll \lambda_{n}\end{cases}
$$


This means that it is not worth considering candidates of $\gamma$ outside of the interval $\left[\lambda_{1}, \lambda_{n}\right]$. In fact, when $\gamma \in\left[\lambda_{1}, \lambda_{n}\right]$ approaches either $\lambda_{1}$ or $\lambda_{n}$, the performance of RLS is likely to suffer from under- or overfitting, respectively. It is worth mentioning that there are ways of bounding $\lambda_{1}$ and $\lambda_{n}$ when calculating them is prohibitive (see Wolkowicz and Styan, 1980, and references therein).

\subsubsection{Challenges in RLS Parameter Selection}

Formally, the best choice of $k$ and $\gamma$ is the one that yields in Expression (6.2) a function $f_{k, \gamma}$ that minimizes the risk of prediction error as measured by the expected squared loss, which is given by

$$
\mathcal{R}(f)=\int(y-f(\boldsymbol{x}))^{2} d P(\boldsymbol{x}, y)
$$

The minimum of the functional $\mathcal{R}(f)$ is attained at the regression function (Vapnik, 1998, Chapter 1). Thus, the closer $\mathcal{R}\left(f_{k, \gamma}\right)$ is to the minimum of $\mathcal{R}(f)$, the closer $f_{k, \gamma}$ is to the real regression function in the norm $L^{2}(P)$.

The choice of suitable $k$ and $\gamma$ belongs to the category of problems known as parameter (or model) selection. In contrast with the related category of model assessment, parameter selection does not require the estimation of the value of the prediction error $\mathcal{R}(f)$. It suffices to indicate the function with the smallest $\mathcal{R}\left(f_{i}\right)$ among a set of candidate functions $f_{1}, f_{2}, \ldots, f_{N}$.

In practice, the value of $\mathcal{R}(f)$ cannot be calculated because $P(\boldsymbol{x}, y)$ is unknown. A widely employed workaround in this case is to use available data in a validation setting, that is, to use some portion of the data to perform the minimization of Expression (6.2) for several candidates of $k$ and $\gamma$, and to hold out the other portion for approximating $\mathcal{R}(f)$ and selecting the best $k$ and $\gamma$.

Implementing leave-one-out cross-validation (LOOCV) in RLS is relatively inexpensive compared to other learning methods, and this corresponds to the most interesting property of the method. Nevertheless, conducting LOOCV in RLS is not a panacea. For small training sets, the combination of cross-validation procedures and large sets of admissible functions may lead to overfitting.

In order to tackle this issue, we investigate alternative, statistical or heuristic, procedures for parameter selection. The procedures we consider were shown to perform well for other regression methods, and, to the best of our knowledge, have not been applied to RLS before. In another front, we follow Izmailov et al. (2013) and investigate the use of the INK-splines kernel in regression estimation. We go one step further in this work and propose the use of additive INK-splines kernels instead of multiplicative ones, what may help improve performance for small training sets. 


\subsection{Statistical Parameter Selection}

In this section, we focus on statistical procedures of parameter selection. These methods can be used to bound or estimate the value of the prediction error $R(f)$ - Expression (6.9). Although these statistical procedures can be used to perform model assessment, remind that only their parameter selection capabilities are of interest in this work.

\subsubsection{Leave-One-Out Cross-Validation}

Cross-validation (CV) is one of the earliest parameter selection/assessment techniques that appeared in machine learning (Luntz and Brailovsky, 1969) and is still the most used one. The idea is to allow the same dataset to be used for learning and evaluation.

Here we focus on the special case of leave-one-out cross-validation, which in its $j$-th iteration uses all training points except the $j$-th one to learn a function $f^{j}(\boldsymbol{x})$, and after that evaluates the prediction error of $f^{j}(\boldsymbol{x})$ on the $j$-th point. After iterating over all $n$ training points, the following estimate of the prediction error of the learning algorithm can be calculated:

$$
\mathcal{R}_{\text {loo }}(f)=\frac{1}{n} \sum_{j=1}^{n}\left(y_{j}-f^{j}(\boldsymbol{x})\right)^{2} .
$$

Under the assumption that the functions $f^{j}(\boldsymbol{x})$ are not very different from the function $f(\boldsymbol{x})$ learned using all training data (i.e, the learning algorithm is stable), then $\mathcal{R}_{\text {loo }}(f)$ is an "almost" unbiased estimate of $\mathcal{R}(f)$ (Luntz and Brailovsky, 1969; Elisseeff and Pontil, 2002). This way, among a set of candidate functions, the leave-one-out procedure selects the function which minimizes $\mathcal{R}_{l o o}(f)$.

In general, the major drawback of leave-one-out $\mathrm{CV}$ is the requirement to call the learning algorithm as many times as the number of training points. Fortunately, the special structure of the regularized least squares method allows the calculation of $\mathcal{R}_{\text {loo }}\left(f_{k, \gamma}\right)$ practically for free after finding the solution $\boldsymbol{\alpha}_{\gamma}$ in Expression (6.8) using all training data and the kernel matrix $K$.

The following derivations are carried out when the $j$-th example is held-out. Let $f_{k, \gamma}^{j}$ be the function obtained through Expression (6.8) using the $n-1$ remaining points

$$
f_{k, \gamma}^{j}(\boldsymbol{x})=\sum_{i=1}^{n} \alpha_{\gamma, i}^{j} k\left(\boldsymbol{x}_{i}, \boldsymbol{x}\right)
$$

The following proposition regarding $f_{k, \gamma}^{j}$ is true (Wahba, 1990; Rifkin, 2006).

Proposition 6.1. Let $f_{k, \gamma}^{j}$ be the minimizer of the $R L S$ problem

$$
\min _{f \in \mathcal{F}_{K}}\left[\frac{1}{n} \sum_{i=1, i \neq j}^{n}\left(y_{i}-f\left(\boldsymbol{x}_{i}\right)\right)^{2}+\gamma\|f\|_{K}^{2}\right] .
$$


Then, $f_{k, \gamma}^{j}$ is also the minimizer of the RLS problem

$$
\begin{aligned}
\min _{f \in \mathcal{F}_{K}}\left[\frac{1}{n} \sum_{i=1, i \neq j}^{n}\left(y_{i}-f\left(\boldsymbol{x}_{i}\right)\right)^{2}\right. & \\
& \left.+\frac{1}{n}\left(f_{k, \gamma}^{j}\left(\boldsymbol{x}_{j}\right)-f\left(\boldsymbol{x}_{j}\right)\right)^{2}+\gamma\|f\|_{K}^{2}\right]
\end{aligned}
$$

Denoting by $\boldsymbol{y}^{j}$ the $n \times 1$ vector $\left[y_{1}^{j}, \ldots, y_{n}^{j}\right]^{\top}$ in which

$$
y_{i}^{j}=\left\{\begin{array}{cc}
y_{i} & i \neq j \\
f_{k, \gamma}^{j}\left(\boldsymbol{x}_{j}\right) & i=j
\end{array},\right.
$$

and using Expression (6.12), the coefficients $\boldsymbol{\alpha}_{\gamma}^{j}$ in Expression (6.11) would be calculated by solving $\boldsymbol{\alpha}_{\gamma}^{j}=(K+\gamma I)^{-1} \boldsymbol{y}^{j}$. However, $\boldsymbol{y}^{j}$ is not fully specified because it depends on the knowledge of $f_{k, \gamma}^{j}$ (and $\boldsymbol{\alpha}_{\gamma}^{j}$ thereof). Fortunately, it is possible to avoid this circular dependence. For this, let $f_{k, \gamma}\left(\boldsymbol{x}_{j}\right)$ be the function obtained through Expression (6.8) using all training points. Denoting $G=(K+\gamma I)$, the following chain of equalities is true:

$$
\begin{aligned}
f_{k, \gamma}^{j}\left(\boldsymbol{x}_{j}\right)-f_{k, \gamma}\left(\boldsymbol{x}_{j}\right) & =\sum_{i=1}^{n}\left(K G^{-1}\right)_{j i}\left(y_{i}^{j}-y_{i}\right) \\
& =\left(K G^{-1}\right)_{j j}\left(f_{k, \gamma}^{j}\left(\boldsymbol{x}_{j}\right)-y_{j}\right) .
\end{aligned}
$$

Solving for $f_{k, \gamma}^{j}\left(\boldsymbol{x}_{j}\right)$ in Expression (6.13) and letting $h_{j j}=\left(K G^{-1}\right)_{j j}$, the following expression for $f_{k, \gamma}^{j}\left(\boldsymbol{x}_{j}\right)$ is obtained:

$$
f_{k, \gamma}^{j}\left(\boldsymbol{x}_{j}\right)=\frac{f_{k, \gamma}\left(\boldsymbol{x}_{j}\right)-h_{j j} y_{j}}{1-h_{j j}} .
$$

Denoting by $g_{i j}^{-1}$ the entries of $G^{-1}$, and after some algebra in Expression (6.14), we arrive at the following expression for the difference between the target value $y_{j}$ and the predicted value by the leave-one-out function $f_{k, \gamma}^{j}\left(\boldsymbol{x}_{j}\right)$ :

$$
y_{j}-f_{k, \gamma}^{j}\left(\boldsymbol{x}_{j}\right)=\frac{\alpha_{j, \gamma}}{g_{j j}^{-1}}
$$

Plugging Expression (6.15) into Expression (6.10), we arrive at the leave-one-out estimator for RLS:

$$
\mathcal{R}_{\text {loo }}\left(f_{k, \gamma}\right)=\frac{1}{n} \sum_{j=1}^{n}\left(\frac{\alpha_{j, \gamma}}{g_{j j}^{-1}}\right)^{2} .
$$

Note that the above expression should be minimized over different candidate pairs of kernel functions and $\gamma$ values. 


\subsubsection{Complexity Penalization}

Another direction of research in statistical parameter selection is based on the idea of bounding the prediction error in Expression (6.9). Let us suppose that a learning algorithm selects from a set of functions $\mathcal{F}$ a function $f$ which minimizes the empirical prediction error (squared error) over $\mathcal{F}$, which is given by

$$
\mathcal{R}_{\text {emp }}(f)=\frac{1}{n} \sum_{i=1}^{n}\left(y_{i}-f\left(\boldsymbol{x}_{i}\right)\right)^{2} .
$$

Moreover, suppose there is a quantity $\mathcal{P}(n, p)$ which measures the degree of capacity (or diversity) of the set of functions $\mathcal{F}$. This function $\mathcal{P}(n, p)$ depends on the size of the training data $n$ and on a parameter of complexity $p$. For instance, in polynomial regression, $p$ would be set to the degree of the polynomial used to fit a set of data.

In what follows, we consider bounds of the form:

$$
\mathcal{R}(f) \leq \mathcal{R}_{e m p}(f) \mathcal{P}(n, p)
$$

This expression captures the idea that the more complex a set of functions $\mathcal{F}$ is, the less we can trust the empirical prediction error $\mathcal{R}_{e m p}(f)$.

In the 1970's, several attempts were made at defining $\mathcal{P}(n, p)$, resulting in parameter selection procedures that were proven to work asymptotically $(n \rightarrow \infty)$. In Table 6.1, the most common definitions are listed ${ }^{1}$. The parameter $p$ present in these definitions is meant to quantify the degrees of freedom of the set of functions $\mathcal{F}$ from which $f$ is selected. We will come back to $p$ at the end of this section.

In the 1990's, a non-asymptotic expression for $\mathcal{P}(n, p)$ was derived based on the results of the VC-Theory (Vapnik, 1998). The general form of this expression is ${ }^{2}$

$$
\mathcal{P}(n, p)=\left(1-c \sqrt{\frac{p\left(1+\ln \frac{a n}{p}\right)-\ln \eta}{n}}\right)_{+}
$$

where $p$ corresponds to the VC-dimension of the set of functions $\mathcal{F}$ and $c, a, \eta$ are constants that need to be set to reflect the particularities of the learning method being used. In (Cherkassky et al., 1999), the assignment $c=a=1$ and $\eta=1 / \sqrt{n}$ was shown to perform well in a polynomial regression task.

The Effective Number of Parameters in RLS. One of the tenets of Statistical Learning Theory (Vapnik, 1998) is that the VC-dimension is the characterization of the complexity

\footnotetext{
${ }^{1}$ Unlike its name suggests, the GCV criterion is not a cross-validation procedure, but actually an approximation to the leave-one-out estimator given by Expression (6.10)

${ }^{2}(a)_{+}=\max (0, a)$
} 
Table 6.1: Classical complexity penalization (Vapnik, 1998, Section 13.1.2)

\begin{tabular}{lc}
\hline Name & $\mathcal{P}(n, p)$ \\
\hline Final Prediction Error (FPE) (Akaike, 1970) & $\frac{1+\frac{p}{n}}{1-\frac{p}{n}}$ \\
Generalized Cross-Validation (GCV) (Wahba and Craven, 1979) & $\frac{1}{\left(1-\frac{p}{n}\right)^{2}}$ \\
Schwartz Criterion (SC) (Schwarz, 1978) & $1+\frac{\frac{p}{n} \ln n}{2\left(1-\frac{p}{n}\right)}$ \\
Shibata's Model Selector (SMS) (Shibata, 1981) & $1+2 \frac{p}{n}$ \\
\hline
\end{tabular}

(capacity) of a set of functions $\mathcal{F}$. However, in the case of the sets of functions in RLS, it is not known how to efficiently calculate their VC-dimension. When $\mathcal{F}$ is a set of functions linear in their parameters, which is the case of RLS, the heuristic method for calculating the effective number of parameters (Hastie and Tibshirani, 1990) can be used as a proxy for the VC-dimension.

Let $\lambda_{1}>\lambda_{2}>\ldots>\lambda_{n}$ be the eigenvalues of the kernel matrix $\mathrm{K}$ used for solving the RLS problem in Expression (6.8). The value

$$
p_{\gamma}=\sum_{i=1}^{n} \frac{\lambda_{i}}{\lambda_{i}+\gamma}, \quad 0<p_{\gamma} \leq n
$$

quantifies the effective degrees of freedom of the set of functions from where the solution $f_{k, \gamma}$ is selected (Vapnik, 1998). Using $p=p_{\gamma}$ in any of the expressions for $\mathcal{P}(n, p)$ shown in this section, we arrive at the following complexity penalization estimator in RLS:

$$
\mathcal{R}_{c p}\left(f_{k, \gamma}\right)=\mathcal{R}_{e m p}\left(f_{k, \gamma}\right) \mathcal{P}\left(n, p_{\gamma}\right)
$$

\subsection{Heuristic Parameter Selection}

In this section we present two metric-based heuristic procedures that have been found to perform well in certain parameter selection tasks. It is not clear how these procedures relate to our goal in Expression (6.9). Hence, their performance need to be experimentally evaluated to have an idea of how promising they are in RLS parameter selection.

Both procedures, called TRI and ADJ, use geometric ideas and unlabeled data to perform parameter selection (Schuurmans, 1997; Schuurmans et al., 2006). In particular, the ADJ heuristic was found to be a state-of-the-art parameter selection procedure for selecting the degree parameter in polynomial regression (Chapelle et al., 2002; Schuurmans et al., 2006). 


\subsubsection{TRI}

Suppose the real regression function $f_{\text {reg }}(x)$ is known and belongs to a metric space. Then, given any two functions $f_{1}(x)$ and $f_{2}(x)$ in the same metric space and the distance (metric) $\rho(\cdot, \cdot)$, the triangle inequality applies:

$$
\rho\left(f_{1}, f_{\text {reg }}\right)+\rho\left(f_{2}, f_{\text {reg }}\right) \geq \rho\left(f_{1}, f_{2}\right) \text {. }
$$

Given a training sequence $\left(\boldsymbol{x}_{1}, y_{1}\right), \ldots,\left(\boldsymbol{x}_{n}, y_{n}\right)$ and a large set of unlabeled data $\boldsymbol{x}_{1}^{*}, \ldots, \boldsymbol{x}_{m}^{*}$ which comes from the same distribution as the training sequence, we can approximately verify the validity of the triangle inequality using

$$
\rho_{t r}\left(f_{1}, \boldsymbol{y}\right)+\rho_{t r}\left(f_{2}, \boldsymbol{y}\right) \geq \rho_{u n}\left(f_{1}, f_{2}\right),
$$

where $\rho_{t r}(f, \boldsymbol{y})=\sqrt{\mathcal{R}_{e m p}(f)}$ and $\rho_{u n}\left(f_{1}, f_{2}\right)$ is the disagreement of functions $f_{1}$ and $f_{2}$ on the unlabeled data:

$$
\rho_{u n}\left(f_{1}, f_{2}\right)=\sqrt{\frac{1}{n} \sum_{i=1}^{m}\left(f_{1}\left(\boldsymbol{x}_{i}^{*}\right)-f_{2}\left(\boldsymbol{x}_{i}^{*}\right)\right)^{2}} .
$$

Now, let us use RLS and a fixed kernel $K$ to obtain two functions $f_{\gamma_{1}}(x)$ and $f_{\gamma_{2}}(x)$ such that $\gamma_{1}>\gamma_{2}$. Consequently, while $f_{\gamma_{2}}(x)$ will potentially have a smaller empirical error than $f_{\gamma_{1}}(x)$, there is a risk that its empirical error is a rough estimate of $\rho\left(f_{1}, f_{\text {reg }}\right)$, and it may happen that:

$$
\rho_{t r}\left(f_{\gamma_{1}}, \boldsymbol{y}\right)+\rho_{t r}\left(f_{\gamma_{2}}, \boldsymbol{y}\right)<\rho_{u n}\left(f_{\gamma_{1}}, f_{\gamma_{2}}\right) .
$$

In this situation, the TRI heuristic blames the function $f_{\gamma_{2}}$ for the violation of the triangle inequality. This gives rise to the TRI procedure for parameter selection: given a sequence of functions $f_{\gamma_{1}}, f_{\gamma_{2}}, \ldots$ such that $\gamma_{1}>\gamma_{2}>\ldots$, choose the last function in the sequence that does not violate the triangle inequality along with any preceding function.

As the TRI method was developed for estimating an ordered parameter, it applies only to the selection of the parameter $\gamma$ of RLS. That is, this method cannot be used for choosing a kernel function.

\subsubsection{ADJ}

This other heuristic is based on the assumption that as the training and unlabeled sets come from the same distribution, the following relation should be observed:

$$
\frac{\rho_{u n}\left(f_{1}, f_{r e g}\right)}{\rho_{u n}\left(f_{1}, f_{2}\right)} \approx \frac{\rho_{t r}\left(f_{1}, f_{r e g}\right)}{\rho_{t r}\left(f_{1}, f_{2}\right)}
$$


If the functions $f_{1}$ and $f_{2}$ are given along with the training and unlabeled sets, the values $\rho_{t r}\left(f_{1}, f_{2}\right)$ and $\rho_{u n}\left(f_{1}, f_{2}\right)$ can be readily calculated. The value $\rho_{t r}\left(f_{1}, f_{\text {reg }}\right)$ can be estimated using $\rho_{t r}\left(f_{1}, \boldsymbol{y}\right)$. The only remaining unknown in Expression $(6.17)$ is $\rho_{u n}\left(f_{1}, f_{\text {reg }}\right)$. Thus, it can be estimated using

$$
\rho_{u n}\left(f_{1}, f_{\text {reg }}\right)=\rho_{t r}\left(f_{1}, \boldsymbol{y}\right) \frac{\rho_{u n}\left(f_{1}, f_{2}\right)}{\rho_{t r}\left(f_{1}, f_{2}\right)} .
$$

This means that, if the assumption in Expression (6.17) is valid, the prediction error of function $f_{1}$ on unseen data, which is a good approximation to the true prediction error, can be estimated from its empirical error $\rho_{t r}\left(f_{1}, \boldsymbol{y}\right)=\sqrt{\mathcal{R}_{e m p}\left(f_{1}\right)}$ and the ratio $\frac{\rho_{u n}\left(f_{1}, f_{2}\right)}{\rho_{t r}\left(f_{1}, f_{2}\right)}$.

This gives rise to the ADJ procedure, which for parameter selection in RLS reads: given a fixed kernel function $k$ and a sequence of RLS functions $f_{\gamma_{1}}=f_{k, \gamma_{1}}, f_{\gamma_{2}}=f_{k, \gamma_{2}}, \ldots$ such that $\gamma_{1}>\gamma_{2}>\ldots$, first multiply $\rho_{t r}\left(f_{\gamma_{i}}, \boldsymbol{y}\right)$ by

$$
\mu\left(f_{\gamma_{i}}\right)=\max _{\{j: j<i\}} \frac{\rho_{u n}\left(f_{\gamma_{i}}, f_{\gamma_{j}}\right)}{\rho_{t r}\left(f_{\gamma_{i}}, f_{\gamma_{j}}\right)},
$$

and then choose the function $f_{\gamma_{i}}$ that has the smallest value of

$$
\mathcal{R}_{a d j}\left(f_{\gamma_{i}}\right)=\sqrt{\mathcal{R}_{e m p}\left(f_{\gamma_{i}}\right)} \mu\left(f_{\gamma_{i}}\right) .
$$

Note from Expression (6.19) that the ADJ method resembles a complexity penalization procedure. Unlike TRI, the ADJ method can be used for selecting both $\gamma$ and $k$ by taking the minimum of $\mathcal{R}_{a d j}\left(f_{\gamma_{i}}\right)$ over $k$.

\subsection{Choosing a Kernel Function for RLS}

The choice of a kernel function for regularized least squares defines the set of functions over which the minimization in Expression (6.2) takes place. For example, if a linear kernel $k_{\text {lin }}(\boldsymbol{x}, \boldsymbol{z})=\boldsymbol{x}^{\top} \boldsymbol{z}$ is chosen, the function obtained by RLS will be a hyperplane in the input space, which is enough for learning a linear regression. However, the regression function is not linear in the input space for a variety of practical problems. This is why we have to choose among kernel functions that generate non-linear functions in the input space.

As already mentioned, a typical non-linear kernel is the widely used Gaussian RBF kernel:

$$
k_{r b f}(\boldsymbol{x}, \boldsymbol{z})=\exp \left(-\sigma\|\boldsymbol{x}-\boldsymbol{z}\|_{2}^{2}\right) .
$$

In fact, this expression defines a family of kernel functions parameterized by $\sigma$, the socalled width parameter. By controlling $\sigma$, it is possible to achieve universal approximation of continuous functions (Steinwart, 2001). However, at least two problems occur when 
working with RBF kernels due to this extra parameter: 1) the need for selecting it properly for obtaining good generalization; and 2) the potential for overfitting in small sample situations, as the set of induced functions is very flexible. These problems also affect polynomial kernels, since by choosing to use high-order polynomials (by controlling the degree parameter), one can easily overfit the training data.

The issues plaguing RBF and polynomial kernels have led researchers to carry out regression estimation based on splines (Wahba, 1990). The underlying idea is to approximate a complicated regression locally, using a low-order polynomial in each piece of the function. By doing that, we avoid working with higher-order polynomials, decreasing the risk of overfitting, while retaining good approximation properties.

It is possible to define a kernel for generating splines in one dimension (Vapnik, 1998, Chapter 11). Denoting $x \wedge z=\min (x, z)$, the expression

$$
k_{i n k}(x, z)=1+x z+\frac{1}{2}|x-z|(x \wedge z)^{2}+\frac{1}{3}(x \wedge z)^{3}
$$

defines a spline kernel for positive values of $x$ and $z$, which is named the INK-splines kernel in (Izmailov et al., 2013). Using this kernel, the functions generated by RLS are twice differentiable at the training points.

The problem now is how to extend the one-dimensional INK-splines to the multidimensional case. In (Izmailov et al., 2013), a multidimensional INK-splines kernel was proposed as a multiplication over one-dimensional kernels corresponding to each dimension of the data ${ }^{3}$. The authors also propose normalizing it to ease (but not avoid) numerical difficulties. The expression for the kernel proposed in (Izmailov et al., 2013) is then:

$$
\tilde{k}_{i n k}^{d}(\boldsymbol{x}, \boldsymbol{z})=\prod_{k=1}^{d} \frac{k_{i n k}\left(x^{d}, z^{d}\right)}{\sqrt{k_{i n k}\left(x^{d}, x^{d}\right) k_{i n k}\left(z^{d}, z^{d}\right)}} .
$$

In (Izmailov et al., 2013), the kernel in Expression 6.20 was experimentally evaluated only on SVM classification. In this work, we evaluate it on RLS regression. We also investigate the additive normalized INK-splines kernel, which is a sum over the normalized one-dimensional kernels corresponding to each dimension of the data:

$$
k_{a d d}^{d}\left(\boldsymbol{x}_{i}, \boldsymbol{x}_{j}\right)=\sum_{k=1}^{d} \frac{k_{i n k}\left(x^{d}, z^{d}\right)}{\sqrt{k_{i n k}\left(x^{d}, x^{d}\right) k_{i n k}\left(z^{d}, z^{d}\right)}} .
$$

Using an additive INK-splines kernel as in Expression (6.21) instead of a multiplicative one as in Expression (6.20) is numerically safer. In particular, Expression (6.21) enables us to use a spline kernel in high-dimensions, whereas the value in Expression (6.20) would easily fall out of the precision range of standard floating-point arithmetic.

\footnotetext{
${ }^{3}$ The product of one-dimensional kernels is itself a kernel function (Vapnik, 1998, Section 11.5.2).
} 
Like the additive min kernel of Section 5.3.2, the additive INK-splines kernel has less capacity than the multiplicative INK-splines. This fact may be advantageous when dealing with small training sets. In the next section, we experimentally verify the performance of the additive INK-splines kernel.

\subsection{Experiments}

The experiments reported in this section aim at answering the following questions regarding parameter selection in regularized least squares:

Q1: How the performance of the multiplicative INK-splines kernel compares with that of the RBF kernel? How the performance of these two kernels compares with the additive INK-splines kernel?

Q2: The complexity penalization methods described in Section 6.2.2 were evaluated mainly for polynomial regression in artificial datasets, where they were found to be comparable to cross-validation (Cherkassky et al., 1999). Does this result hold for RLS in real datasets?

Q3: Can the constants in the VC complexity penalization in Expression (6.16) for $P(n, p)$ given in (Cherkassky et al., 1999) be improved for parameter selection in RLS?

Q4: The heuristic approach ADJ was shown to give state-of-the-art results in polynomial regression, even outperforming cross-validation, while TRI has been less successful than ADJ (Chapelle et al., 2002; Schuurmans et al., 2006). Do these results hold for RLS in real datasets?

\subsubsection{Experimental Setup}

We use 10 regression datasets from two repositories: UCI ${ }^{4}$ and Keel ${ }^{5}$ (AlcaláFernández et al., 2011). Table 6.2 provides a description of the datasets.

In what follows, we describe a single trial of our experiments for a fixed dataset. Two-thirds of the dataset were randomly held-out to form a test set $\left(\boldsymbol{x}_{1}^{\prime}, y_{1}^{\prime}\right), \ldots,\left(\boldsymbol{x}_{\ell}^{\prime}, y_{\ell}^{\prime}\right)$. From the remaining one-third, $n$ points are randomly selected to compose the training set $\left(\boldsymbol{x}_{1}, y_{1}\right), \ldots,\left(\boldsymbol{x}_{n}, y_{n}\right)$. The points not selected in the one-third to compose the training set are used without their labels as the unlabeled set $\boldsymbol{x}_{1}^{*}, \ldots, \boldsymbol{x}_{m}^{*}$ for both TRI and ADJ methods.

The training set thus created is used along with a fixed kernel $k$ and a value of $\gamma$ to obtain a function $f_{k, \gamma}$ in accordance to Expression (6.8). The root mean squared error

\footnotetext{
${ }^{4}$ http://archive.ics.uci.edu/ml/

${ }^{5}$ http://sci2s.ugr.es/keel/datasets.php
} 
Table 6.2: Regression datasets description

\begin{tabular}{|c|c|c|c|c|}
\hline DATASET & Description & Instances & Features & Reference \\
\hline Abalone & $\begin{array}{l}\text { Predict the age of abalone from } \\
\text { physical measurements. }\end{array}$ & 4177 & 8 & $\begin{array}{l}\text { http://archive.ics. } \\
\text { uci.edu/ml/datasets/ } \\
\text { Abalone }\end{array}$ \\
\hline COMPACTIV & $\begin{array}{l}\text { Predict the portion of time that } \\
\text { a CPU runs in user mode from } \\
\text { system activity measures }\end{array}$ & 8192 & 21 & $\begin{array}{l}\text { http://www.cs. } \\
\text { toronto.edu/ delve/ } \\
\text { data/comp-activ/ } \\
\text { compActivDetail.html }\end{array}$ \\
\hline Concrete & $\begin{array}{l}\text { Predict concrete compressive } \\
\text { strength from concrete age and } \\
\text { ingredients }\end{array}$ & 1030 & 8 & (Yeh, 1998) \\
\hline FRIEDMAN & $\begin{array}{l}\text { Friedman's benchmark data } \\
\text { set. }\end{array}$ & 1200 & 5 & (Friedman, 1991) \\
\hline MorTGage & $\begin{array}{l}\text { Model weekly mortgage rate us- } \\
\text { ing economic data }\end{array}$ & 1049 & 15 & $\begin{array}{l}\text { http://funapp.cs. } \\
\text { bilkent.edu.tr/ } \\
\text { DataSets/Data/MO. } \\
\text { names }\end{array}$ \\
\hline Stock & $\begin{array}{l}\text { Approximate the stock price } \\
\text { of a company given the stock } \\
\text { prices of other companies. }\end{array}$ & 950 & 9 & $\begin{array}{l}\text { http://sci2s.ugr.es/ } \\
\text { keel/dataset.php?cod= } \\
77\end{array}$ \\
\hline Treasury & $\begin{array}{l}\text { Model weekly rate of Certifi- } \\
\text { cate of Deposits from economic } \\
\text { data. }\end{array}$ & 1049 & 15 & $\begin{array}{l}\text { http://funapp.cs. } \\
\text { bilkent.edu.tr/ } \\
\text { DataSets/Data/TR. } \\
\text { names }\end{array}$ \\
\hline WANKARA & $\begin{array}{l}\text { Predict the mean daily temper- } \\
\text { ature in Ankara, Turkey from } \\
\text { weather information. }\end{array}$ & 321 & 9 & $\begin{array}{l}\text { http://funapp.cs. } \\
\text { bilkent.edu.tr/ } \\
\text { DataSets/Data/WA. } \\
\text { names }\end{array}$ \\
\hline WinE- R & $\begin{array}{l}\text { Model wine quality based on } \\
\text { physicochemical tests. }\end{array}$ & 1599 & 11 & (Cortez et al., 2009) \\
\hline Wine-W & $\begin{array}{l}\text { Model wine quality based on } \\
\text { physicochemical tests. }\end{array}$ & 4898 & 11 & (Cortez et al., 2009) \\
\hline
\end{tabular}

(RMSE) of $f_{k, \gamma(x)}$ in the test set

$$
\operatorname{rmse}\left(f_{\gamma}\right)=\sqrt{\frac{1}{\ell} \sum_{i=1}^{\ell}\left(f_{k, \gamma}\left(\boldsymbol{x}_{i}^{\prime}\right)-y_{i}^{\prime}\right)^{2}}
$$

is then recorded for evaluation purposes.

In order to try reasonable values of $\gamma$, the leading eigenvalue $\lambda_{1}$ of the kernel matrix $K$ is taken as a reference. Using the 50 values $v_{1}, \ldots, v_{50}$ equally spaced according to the logarithmic scale in the range $\left[10^{-6}, 10^{1}\right]$, we try the following 50 values: $\gamma_{1}=v_{1} \lambda_{1}, \ldots, \gamma_{50}=v_{50} \lambda_{1}$.

Thus, after obtaining $f_{k, \gamma_{1}}, \ldots, f_{k, \gamma_{50}}$, and calculating their respective RMSE on the test set, we find the function $f_{k, \gamma_{*}}$ with the minimum value of RMSE, which is the gold standard to our parameter selection experiments described ahead.

For the RBF kernel, we used only leave-one-out cross-validation for selecting $\gamma$ and $\sigma$. 
For selecting a good range of candidates of $\sigma$, we calculated the empirical distribution of the distance between all pairs of training points and then considered 5 percentiles of this distribution as candidates of $\sigma: 5,25,50,75$, and 95 .

For both the additive and the multiplicative INK-splines, we have implemented and run the following parameter selection procedures: leave-one-out cross-validation (LOOCV); complexity penalization: final prediction error (FPE), generalized cross-validation (GCV), Schwarz criterion (SC), Shibata's model selector (SMS), VC bound (VC1); and metricbased: TRI and ADJ. Each parameter selection procedure had access only to the training set, with the exception of TRI and ADJ, which had access to the unlabeled set as well. According to each parameter selection procedure, one function $f_{\text {chosen }}$ was selected among $f_{\gamma_{1}}, \ldots, f_{\gamma_{50}}$. We evaluated the quality of each procedure by the ratio:

$$
r=\frac{r m s e\left(f_{\text {chosen }}\right)}{r m s e\left(f_{\gamma^{*}}\right)}
$$

That is, the closer $r$ is to 1 , the better the parameter selection performance is.

So far, this description refers to a single trial. For a fixed size of the training set $n$, we conducted 20 such trials and report the mean and the standard deviation of each parameter selection procedure. This experiment was carried out for $n=20$ and $n=100$ on each dataset.

In order to address question Q2, the constants $c$ and $a$ in Expression (6.16) were experimentally optimized using only one of the datasets (randomly selected). The resulting constants $c=0.74$ and $a=1.35$ were used as another parameter selection procedure with the $\mathrm{VC}$ expression ( $\mathrm{VC} 2)$.

\subsubsection{Results and Discussion}

We start the analysis of the results by comparing the three kernel functions considered in this work: RBF, multiplicative INK-splines, and additive INK-splines. Table 6.3, in which Mul. Spl. (resp. Add. Spl.) refer to multiplicative (resp. additive) INK-splines, reports:

1. The best RMSE achieved on the test set among $f_{k, \gamma_{1}}, \ldots, f_{k, \gamma_{50}}$ for a fixed kernel $k$;

2. The value of RMSE achieved on the test set by the best LOOCV function among the $f_{k, \gamma_{1}}, \ldots, f_{k, \gamma_{50}}$ for a fixed kernel $k$.

For each dataset and $n$, we highlight in bold the best value of RMSE across the three kernels in the set of columns under "LOOCV", and do the same for the set of columns under "Best". 
Note that the additive INK-splines kernel in combination with LOOCV was the best performing regression estimation method in 8 out of 10 cases when $n=20$. When $n=100$, it was the best in 7 out of 10 cases. Note also the asymmetry of the results: in the few cases where the additive kernel did not provide the best results, the relative difference to the best result is not large; however, when the additive kernel is the best performing method, the relative gain with respect to the other kernels can be substantial. See, for example, the results for datasets COMPACTIV, MorTgage, and Treasury. Interestingly, these three datasets correspond to the ones with the highest number of dimensions among the 10 datasets considered.

Regarding the best possible selection of $\gamma$ (columns grouped under "Best"), observe there are few cases for which the best possible result for the RBF kernel is the best across the three kernels (e.g WANKARA dataset). However, when LOOCV is applied, these results may not hold (see COMPACTIV dataset for $n=20$ ). In the case of the additive INK-splines kernel, if its results happened to the best among the three kernels in "Best", the same could be said for the "LOOCV" results (the exception being the STOCK dataset). In the case of the multiplicative INK-splines kernel, we see that overfitting with LOOCV is not an issue like it is for RBF. However, the best possible result is not so

Table 6.3: Experimental results for the best possible function and the one selected by leave-one-out across different kernel functions.

\begin{tabular}{|c|c|c|c|c|c|c|c|}
\hline \multirow{2}{*}{ DATASET } & \multirow{2}{*}{$n$} & \multicolumn{3}{|c|}{ Best } & \multicolumn{3}{|c|}{ LOOCV } \\
\hline & & $\mathrm{RBF}$ & Mul. Spl. & Add. Spl. & $\mathrm{RBF}$ & Mul. Spl. & Add. Spl. \\
\hline \multirow{2}{*}{ ABALONE } & 20 & $2.61(0.22)$ & $2.56(0.22)$ & $2.49(0.17)$ & $3.18(0.91)$ & $3.07(1.12)$ & $\mathbf{2 . 9 1}(0.70)$ \\
\hline & 100 & $2.26(0.05)$ & $2.25(0.06)$ & $\mathbf{2 . 2 4}(0.05)$ & $2.35(0.18)$ & $\mathbf{2 . 2 7}(0.06)$ & $\mathbf{2 . 2 7}(0.06)$ \\
\hline \multirow[b]{2}{*}{ COMPACTIV } & 20 & $10.9(1.60)$ & $74.4(0.99)$ & $12.1(2.91)$ & $16.6(11.8)$ & $74.4(0.99)$ & $12.5(2.76)$ \\
\hline & 100 & $7.49(0.65)$ & $54.3(1.01)$ & $4.07(0.34)$ & $14.8(18.5)$ & $54.3(1.01)$ & $\mathbf{4 . 2 7}(0.43)$ \\
\hline \multirow[b]{2}{*}{ Concrete } & 20 & $11.1(1.42)$ & $16.0(3.08)$ & $\mathbf{9 . 6 3}(1.15)$ & $12.6(2.27)$ & $16.1(3.10)$ & $10.7(2.42)$ \\
\hline & 100 & $8.90(0.42)$ & $8.66(0.85)$ & $7.17(0.31)$ & $9.70(1.59)$ & $8.83(0.90)$ & $7.29(0.33)$ \\
\hline \multirow{2}{*}{ FRIEDMAN } & 20 & $2.84(0.24)$ & $2.90(0.23)$ & $\mathbf{2 . 2 8}(0.24)$ & $3.16(0.31)$ & $3.20(0.32)$ & $\mathbf{2 . 4 9}(0.32)$ \\
\hline & 100 & $\mathbf{1 . 5 5}(0.11)$ & $1.66(0.11)$ & $1.84(0.06)$ & $\mathbf{1 . 5 7}(0.12)$ & $1.68(0.12)$ & $1.88(0.11)$ \\
\hline \multirow{2}{*}{ MoRTGAGE } & 20 & $0.95(0.31)$ & $0.65(0.54)$ & $\mathbf{0 . 3 7}(0.25)$ & $1.49(0.84)$ & $0.67(0.54)$ & $\mathbf{0 . 3 8}(0.26)$ \\
\hline & 100 & $0.19(0.04)$ & $0.19(0.06)$ & $\mathbf{0 . 1 5}(0.02)$ & $0.21(0.04)$ & $0.20(0.07)$ & $\mathbf{0 . 1 6}(0.03)$ \\
\hline \multirow{2}{*}{ STOCK } & 20 & $2.29(0.49)$ & $2.28(0.44)$ & $\mathbf{2 . 2 3}(0.41)$ & $2.42(0.51)$ & $\mathbf{2 . 3 8}(0.52)$ & $2.40(0.42)$ \\
\hline & 100 & $1.22(0.10)$ & $\mathbf{1 . 2 1}(0.12)$ & $1.45(0.14)$ & $1.36(0.33)$ & $\mathbf{1 . 2 9}(0.18)$ & $1.49(0.16)$ \\
\hline \multirow{2}{*}{ TrEAsury } & 20 & $1.10(0.37)$ & $0.79(0.50)$ & $\mathbf{0 . 5 4}(0.25)$ & $1.70(0.75)$ & $0.82(0.49)$ & $\mathbf{0 . 5 8}(0.30)$ \\
\hline & 100 & $0.29(0.03)$ & $0.29(0.04)$ & $\mathbf{0 . 2 6}(0.03)$ & $0.32(0.05)$ & $0.30(0.04)$ & $\mathbf{0 . 2 7}(0.03)$ \\
\hline \multirow{2}{*}{ WANKARA } & 20 & $\mathbf{2 . 5 2}(0.62)$ & $4.16(0.70)$ & $2.69(0.51)$ & $2.89(1.00)$ & $4.20(0.70)$ & $2.91(0.67)$ \\
\hline & 100 & $\mathbf{1 . 4 5}(0.09)$ & $2.45(0.30)$ & $1.62(0.11)$ & $\mathbf{1 . 5 3}(0.15)$ & $2.52(0.33)$ & $1.66(0.13)$ \\
\hline \multirow{2}{*}{ WinE-R } & 20 & $0.91(0.16)$ & $0.89(0.05)$ & $\mathbf{0 . 7 7}(0.04)$ & $1.09(0.34)$ & $0.93(0.07)$ & $\mathbf{0 . 8 7}(0.16)$ \\
\hline & 100 & $0.74(0.03)$ & $0.74(0.03)$ & $\mathbf{0 . 6 9}(0.02)$ & $0.79(0.10)$ & $0.74(0.03)$ & $\mathbf{0 . 6 9}(0.02)$ \\
\hline \multirow{2}{*}{ WinE-W } & 20 & $0.94(0.09)$ & $0.94(0.08)$ & $\mathbf{0 . 8 6}(0.04)$ & $1.07(0.27)$ & $0.98(0.12)$ & $\mathbf{0 . 9 2}(0.11)$ \\
\hline & 100 & $0.81(0.02)$ & $0.81(0.02)$ & $\mathbf{0 . 7 8}(0.02)$ & $0.83(0.03)$ & $0.82(0.03)$ & $\mathbf{0 . 8 0}(0.02)$ \\
\hline \multirow{2}{*}{ Avg. } & 20 & $3.62(0.55)$ & $10.6(0.68)$ & $\mathbf{3 . 3 9}(0.59)$ & $4.62(1.90)$ & $10.7(0.80)$ & $\mathbf{3 . 6 7}(0.81)$ \\
\hline & 100 & $2.49(0.15)$ & $7.25(0.26)$ & $\mathbf{2 . 0 3}(0.11)$ & $3.35(2.11)$ & $7.29(0.28)$ & $2.08(0.13)$ \\
\hline
\end{tabular}


good compared to the additive INK-splines kernel. For the COMPACTIV dataset, we may attribute this result to numerical problems with the multiplicative INK-splines (given the catastrophical result in this dataset). For the other datasets, though, we believe that the better performance of the additive kernel stems from the fact that it provides a set of admissible functions with less capacity than the other kernels provide, yet with enough capacity to avoid underfitting.

We now proceed to the analysis for answering questions 2 through 4 . To this end, we compare $\gamma$ selection procedures individually for the multiplicative INK-splines kernel and then for the additive INK-splines kernel.

Table 6.4 shows the results for the multiplicative INK-splines kernel. The best results for each dataset and $n$ are highlighted in bold, and the second best results (provided they are less than 1.15) are underlined. Taking into account all the results, the following distribution can be observed for $(<$ best $>,<$ 2nd best $>)$ - see also Figure 6.1: LOOCV $(\mathbf{1 5}, \underline{2}) ; \operatorname{FPE}(\mathbf{2}, \underline{8}) ; \operatorname{SC}(\mathbf{2}, \underline{9}) ; \operatorname{GCV}(\mathbf{1}, \underline{3}) ; \operatorname{SMS}(\mathbf{4}, \underline{2}) ; \operatorname{VC} 1(\mathbf{0}, \underline{1}) ; \operatorname{VC} 2(\mathbf{4}, \underline{2}) ; \operatorname{TRI}(\mathbf{5}, \underline{2}) ;$ and ADJ $(\mathbf{4}, \underline{2})$. According to this criteria, it is clear that LOOCV is the best parameter selection procedure overall. However, observe that, for the ABALONE dataset with $n=20$, the leave-one-out procedure chose a parameter that performs much worse than the best one, confirming that this procedure might fail in small sample situations.

Table 6.5 shows the results for the additive INK-splines kernel. As previously, taking into account all the results, the following distribution of $(<$ best $>,<\underline{\text { nd best }}>$ ) can be observed, provided that the second best result is less than 1.15 - see also Figure 6.2: $\operatorname{LOOCV}(\mathbf{7}, \underline{9}) ; \operatorname{FPE}(\mathbf{9}, \underline{4}) ; \operatorname{SC}(\mathbf{6}, \underline{7}) ; \operatorname{GCV}(\mathbf{4}, \underline{1}) ; \operatorname{SMS}(\mathbf{5}, \underline{3}) ; \operatorname{VC} 1(\mathbf{0}, \underline{0}) ; \operatorname{VC} 2(\mathbf{4}, \underline{2}) ; \operatorname{TRI}(\mathbf{5}, \underline{0})$; and ADJ $(\mathbf{3}, \underline{3})$. According to this criteria, it is clear that LOOCV and FPE are the best parameter selection procedures, followed by SC. Again, for the ABALONE dataset with $n=20$, the leave-one-out procedure did not perform as well as it performed for the other datasets.

Table 6.6 summarizes the occurrence of poor results from both experiments (Tables 6.4 and 6.5 ), in the form $(N, r)$, where $N$ is the number of cases (maximum of 20) in which the ratio in Expression (6.22) is greater than or equal to 1.15, and $r$ is the worst ratio obtained among the $N$ cases. As can be observed, there is an improvement in LOOCV when using additive INK-splines. Although it continues showing one bad result, the value of $r$ decreased from 1.20 to 1.17. For FPE and SC the improvement is notorious. FPE goes from $(3,1.54)$ down to $(1,1.19)$, thus showing only one poor case when using the additive kernel and decreasing the worst ratio from 1.54 to 1.19. Similarly, SC goes from $(3,1.54)$ down to $(2,1.17)$. These figures show that not only does the additive spline kernel improve upon the other kernels, but it also helps improve the performance of $\gamma$ selection. 


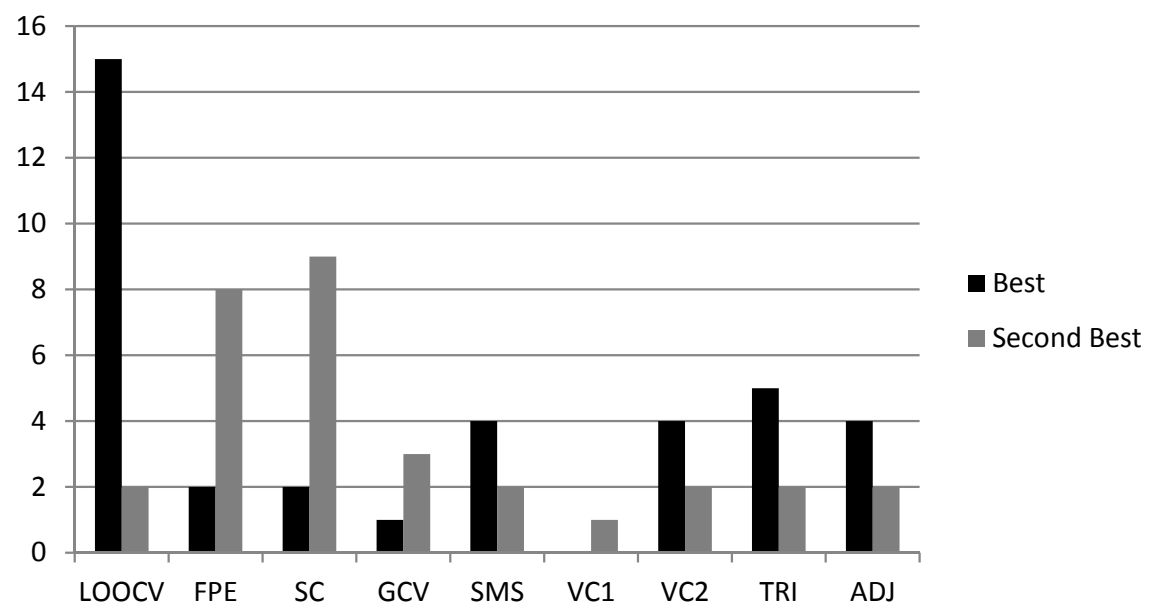

Figure 6.1: Distribution of best results for multiplicative INK-splines.

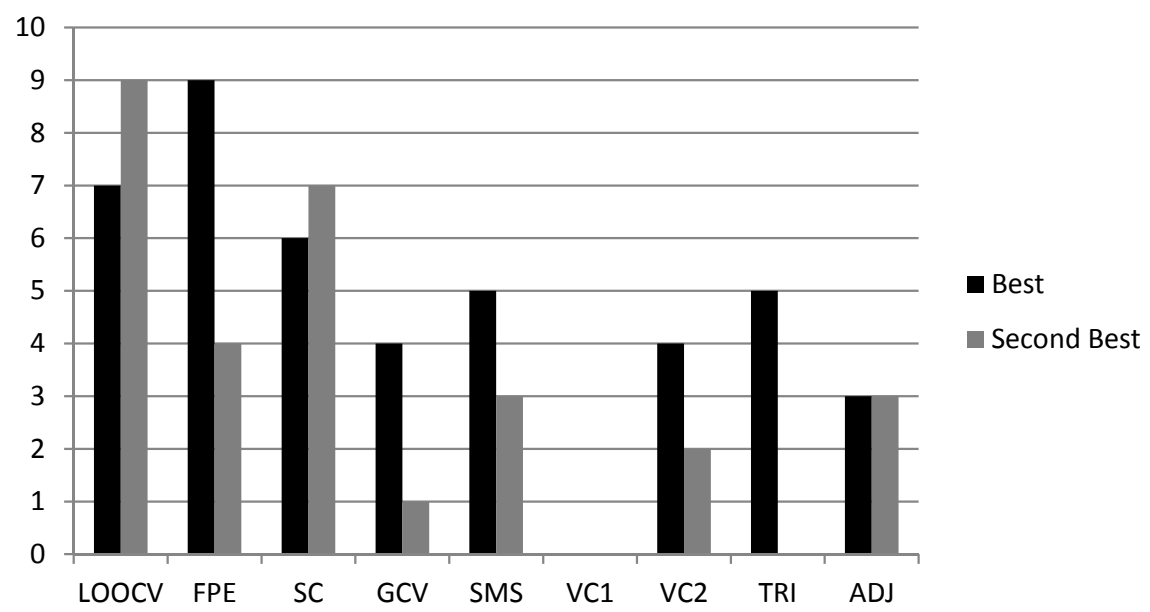

Figure 6.2: Distribution of best results for additive INK-splines. 


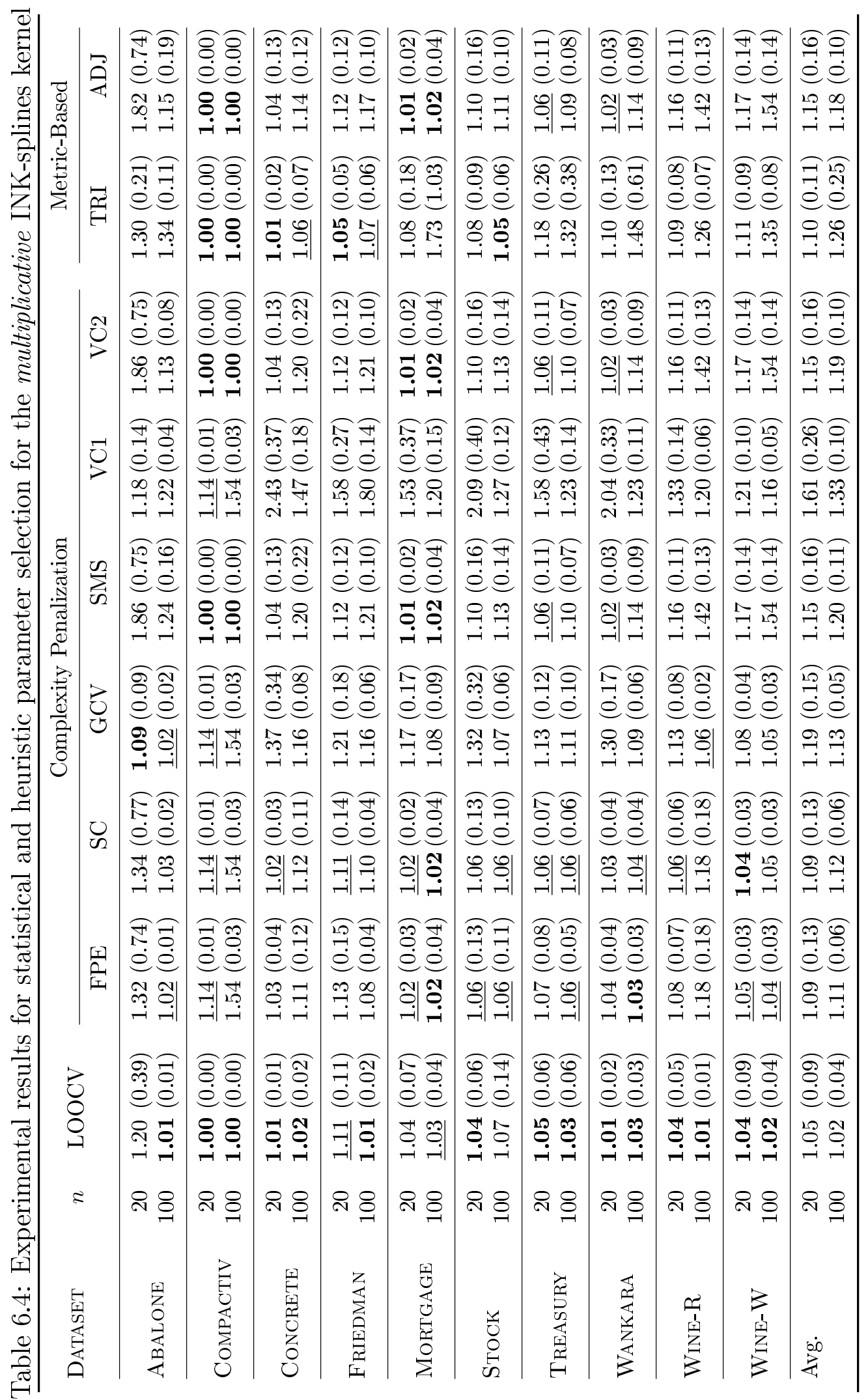




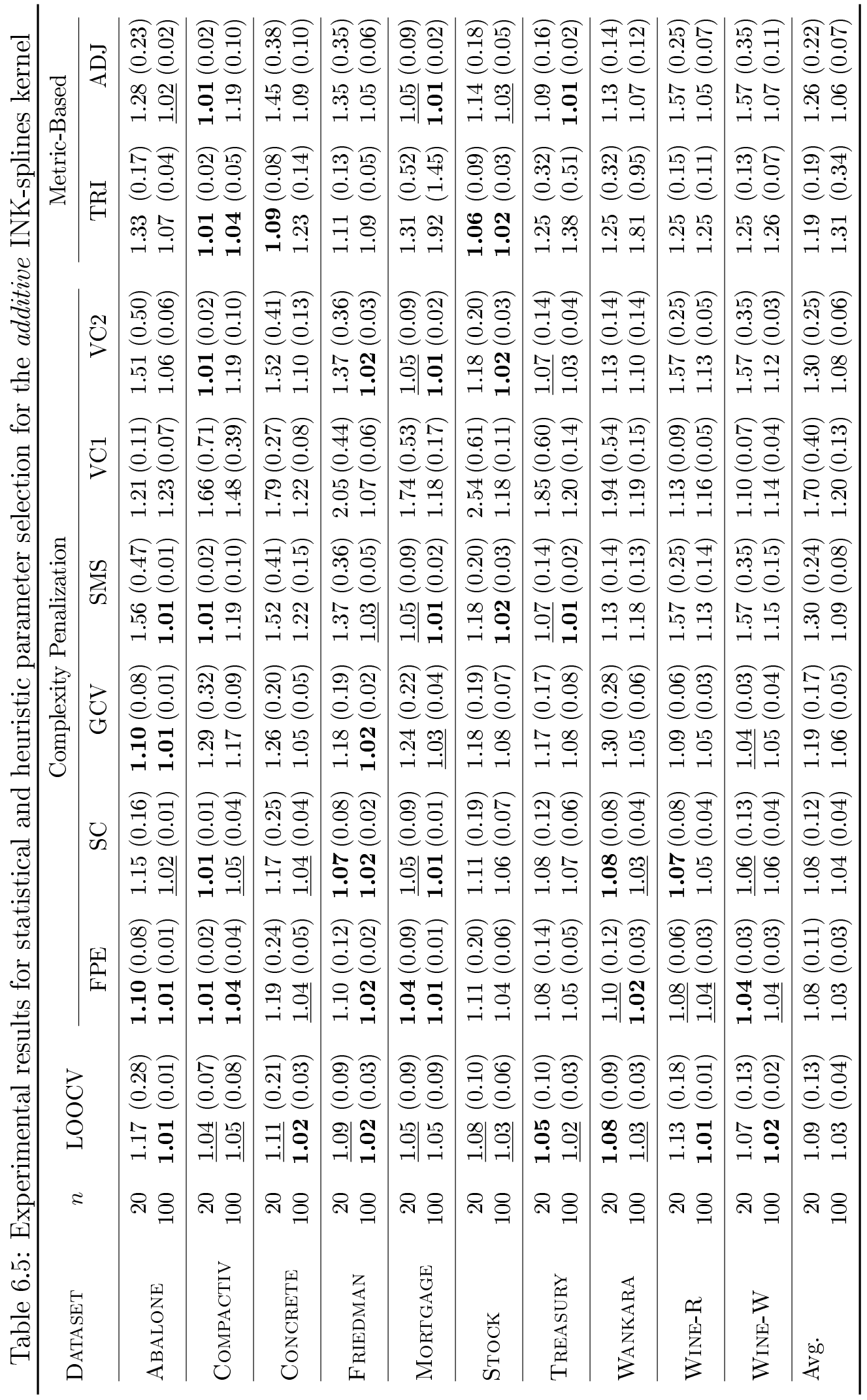


Table 6.6: Summary of poor results for each INK-splines kernels

\begin{tabular}{|c|c|c|c|c|c|c|c|c|c|}
\hline \multirow{2}{*}{ INK-Splines } & \multirow{2}{*}{ LOOCV } & \multicolumn{6}{|c|}{ Complexity Penalization } & \multicolumn{2}{|c|}{ Metric-Based } \\
\hline & & FPE & $\mathrm{SC}$ & $\mathrm{GCV}$ & SMS & $\mathrm{VC} 1$ & $\mathrm{VC} 2$ & TRI & ADJ \\
\hline Multiplicative & $(1,1.20)$ & $(3,1.54)$ & $(3,1.54)$ & $(8,1.54)$ & $(8,1.86)$ & $(18,2.43)$ & $(7,1.86)$ & $(8,1.73)$ & $(7,1.82)$ \\
\hline Additive & $(1,1.17)$ & $(1,1.19)$ & $(2,1.17)$ & $(7,1.26)$ & $(10,1.57)$ & $(16,2.54)$ & $(7,1.57)$ & $(11,1.33)$ & $(5,1.57)$ \\
\hline
\end{tabular}

In what follows we focus on the remaining questions posed in the beginning of this section.

Q2: Considering the complexity penalization procedures (except VC2 which will be treated latter), it can be observed that FPE and SC show similar results, with a slight advantage to the former. Both are better suited to RLS parameter selection than GCV, SMS and VC1. FPE is a competitive alternative to LOO only in conjunction with the additive INK-splines kernel.

Q3: Comparing the results obtained by $\mathrm{VC} 1$ and $\mathrm{VC} 2$, (the last with $c=0.74$ and $a=1.35$ ), better results are obtained by VC2 in most of the cases considered. Moreover, VC1 showed several cases of catastrophic performances. Thus, we can conclude that there is room for improving the constants of the $\mathrm{VC}$ expression for $P(n, p)$ given in (Cherkassky et al., 1999).

Q4: Comparing the results obtained by the two heuristic metric-based approaches, TRI and ADJ, there is no strong pattern of one heuristic outperforming the other for multiplicative INK-splines. For the additive INK-splines, the ADJ method is clearly better for $n=100$, whereas for $n=20$ there is no clear performance distinction between the methods. Thus, these results corroborate partially previous results in polynomial regression (Chapelle et al., 2002; Schuurmans et al., 2006). For the additive INK-splines in particular, the reverse seems to be the case. Comparing TRI and ADJ with LOOCV, worse results are obtained by TRI in most cases, while ADJ performs reasonably well against LOOCV only for $n=100$ when using the additive INK-splines kernel. Even then, the LOOCV method tends to show better performance than ADJ.

\subsection{Final Remarks}

In this chapter, we investigated the parameter selection problem in the regularized least squares method, which narrows down to the selection of a kernel function and a regularization parameter.

As for the selection of the kernel function, we proposed an additive multidimensional INK-splines kernel that outperformed the traditional RBF kernel, as well as the previously 
proposed multiplicative INK-splines kernel. This perceived advantage stems from the fact that the additive INK-splines kernel provides a set of admissible functions with less capacity than the other kernels provide, yet with enough capacity to avoid underfitting.

It is interesting to compare this result for additive INK-splines with the ones in classification using small training sets - Section 5.4.3. Recall from Chapter 5 that the additive min kernel does not always improve the results when using small training sets. In RLS regression, however, replacing multiplication with addition was shown to be very important for improving performance. We believe that the loss functions used in each learning task play a distinctive role in these results. As the RLS loss function, namely the squared error, is more susceptible to noise than the SVM loss function, the lack of training data in RLS highlighted the importance of using kernel functions that generate smaller (less diverse) sets of admissible functions.

Regarding the selection regularization parameter $\gamma$, alternative statistical and heuristic parameter selection procedures were investigated for its selection. These procedures, six of them based on complexity penalization (FPE, SC, GCV, SMS, VC1 and VC2) and two of them based on the geometry of metric spaces (TRI and ADJ), were experimentally evaluated in real datasets and compared to the traditional leave-one-out cross-validation procedure. The results showed that the considered procedures often perform worse than cross-validation. This outcome is different from previous findings in parameter selection for other regression methods.

Our results corroborated that cross-validation may perform poorly when data is scarce, mainly when used in conjunction with RBF kernels. Unfortunately, the best performing alternative method - FPE - does not provide improvements when cross-validation fails. In future work, we plan to investigate ways of combining FPE and LOO that may lead to an improved parameter selection method. 


\section{Chapter 7}

\section{Conclusion}

In this work, we have cast density ratio estimation as the problem of using data to solve the integral equation that defines the density ratio function:

$$
\int_{-\infty}^{x} r(t) d F_{2}(t)=F_{1}(x)
$$

As the left- and right-hand side of this equation are taken approximately, the solution is constructed using instances of the regularization method for stochastic ill-posed problems - Chapter 2.

Two of these instances were considered in Chapter 3: DRE-V and DREV-VK. Using synthetic data, both methods were shown to outperform previously proposed methods KMM (Gretton et al., 2009), uLSIF (Kanamori et al., 2009), and KLIEP (Sugiyama et al., 2008). Moreover, the computational complexity of the methods introduced in this work is not greater than the computational complexity of previous methods.

In the realm of applications - Chapter 4 - , the improvements on density ratio estimation brought by the new methods were also helpful on improving mutual information estimation in classification tasks. VMI, the estimator for mutual information proposed in this work and based on DRE-V, outperformed previous estimators such as Edgeworth (van Hulle, 2005), $k$-NN (Kraskov et al., 2004), and MLMI (Suzuki et al., 2009) on synthetic data.

The improvements in mutual information estimation resulted in improvements in feature selection as well. Our feature selection scheme based on mutual information estimation - VMI + JMI - outperformed previous methods in combination with JMI, with the exception of the $k$-NN estimator. However, comparing $k$-NN and VMI directly ended up being problematic: the parameter $k$ of $k$-NN was selected to optimize feature selection, whereas the regularization parameter $\gamma$ of VMI was selected to optimize density ratio and mutual information estimation. VMI also outperformed the popular feature selection algorithm Relief-F (Kononenko, 1994). 
From the aforementioned work on density ratio estimation and its application to feature selection, two technical questions spun off contributions of independent interest. Both contributions were in the area of parameter selection in learning algorithms employing kernel functions, namely Support Vector Machines (SVM) and Regularized Least Squares (RLS).

In Chapter 5, we developed economic parameter selection procedures for Support Vector Machines that are more reliable than using "default" parameters. The procedure for selecting the generalization parameter $C$ is applicable to any normalized parameterless kernel. For Gaussian RBF kernels, which add another parameter, $\sigma$, to be selected, we developed a specific procedure for considering only a few candidates of $\sigma$. Non-expert SVM users may extract reasonable performance from SVM by following the parameter selection procedures proposed in this work (see Appendix A.1 for a description on how to implement such a procedure in the WEKA framework).

Still in Chapter 5, we proposed the min kernel as a compromise solution to the kernel selection problem, which alleviates even more the burden on the user. The min kernel shares advantages with both linear and non-linear parameterless kernels: it is as suitable to high-dimensional classification as the linear kernel, with the advantage of being nonlinear, so it is also expected to work well in low-dimensional situations.

For the Regularized Least Squares regression method, we focused on parameter selection for small training sets — Chapter 6 - , a situation that may lead the leave-oneout cross-validation (LOOCV) procedure to select bad parameters. After searching and evaluating promising alternative procedures for parameter selection, we concluded that no single alternative procedure could always improve on LOOCV when the latter fails. However, by introducing the additive INK-splines kernel, we were able to mitigate this problem in other front, as this kernel was shown to outperform both the RBF and the multiplicative INK-splines kernel.

In order to further contribute to the topics covered in this work, we list important directions for future research within each chapter.

Chapter 2: it was a choice to follow Tikhonov's regularization method in this work, yet there are other regularization approaches available. For instance, adopting Morozov's regularization method would change the regularization focus to the distribution of the deviations between the left- and right-hand side of the integral equation defining the ratio. This requires theoretical investigation of limiting distributions (empirical processes), something akin to the $\omega^{2}$ square statistic or the functional central limit theorem (Donsker and related theorems). To be useful, such analysis needs to be tailored to the stochastic density ratio estimation problem.

Chapter 3: preliminary experiments with some advanced norms, regularizers, and $\gamma$ selection procedures did not provide consistent improvements over the basic instan- 
tiations. This is not to say that better instantiations do not exist. For example, it would be interesting to experiment with the $L^{1}$ norm for defining the $\rho_{2}$ distance, as well as verify whether a different objective function in the $\gamma$ selection procedure could provide better results. In the later case, it would be interesting to investigate how $\rho_{2}$ would fare in comparison to the least squares criterion.

Chapter 4: supposing we can improve MI estimation for low-dimensional projections of the data, the important question that remains to be answered is how we can best use it to leverage feature selection. In this regard, better selection strategies than JMI are in order. Another important vein is to make practical MI estimation based on density ratios when $y \in \boldsymbol{R}$ (regression case).

Chapter 5: the experimental results in this chapter show that there is room for improving the lower bound of $C_{\text {best }}$. A possible direction of research would be to assume once again that the variables are standardized, as in Section 5.3.1, and try to derive a tighter $C_{\text {low }}$ from a formula obtained from the distribution of kernel values $k\left(\boldsymbol{x}_{i}, \boldsymbol{x}_{j}\right)$. This would avoid the need to calculate the kernel value for all pairs of training points before running SVM.

In another front, the Principal Component Analysis technique may help alleviate the limitation of the min kernel illustrated in Figure 5.5(b). Moreover, the eigenvalues corresponding to the principal components may be useful in weighting the contribution of each transformed coordinate in the min sum.

Chapter 6: the LOOCV method is still problematic for small training sets. Future work could investigate whether there is a better practical proxy for complexity in RLS than the effective number of parameters. Combinations of parameter selection procedures are also welcome. For example, we could try to combine LOOCV with FPE when using the additive INK-splines kernel.

Finally, it is very important to extend the application of stochastic density ratio estimation methods to other application domains, like covariate-shift adaptation and changedetection in time series. However, high-dimensional estimation is more prevalent in these domains. We believe this challenge can be taken when the corresponding methods and algorithms are mature enough. The philosophy is to improve density ratio estimation for low dimensional and small sample situations, investigate the properties of the improved estimators, and try to come up with generalizations to high-dimensional cases. 


\section{Appendix A}

\section{Appendix}

\section{A.1 RBF Parameter Selection in WEKA}

The procedure for parameter selection in SVM with RBF kernels proposed in Section 5.3.1 can be carried out using the software package $\mathrm{WEKA}^{1}$ on a wide variety of datasets (including datasets with categorical features, which are automatically transformed into numerical ones by the SMO implementation in WEKA). All it takes for the user, after loading the training and test sets into WEKA, is to choose the meta-classifier GridSearch and set some options. Figure A.1 shows how to configure GridSearch to perform the parameter selection procedure for $\sigma$ and $C$ proposed in this chapter. Among these settings, there is an option that enables data standardization. The downside with GridSearch is that it is not possible to stop the procedure for selecting $C$ when the crossvalidation error goes up. This way, the user has to specify a number $a$ of candidates that should be investigated beyond $C_{l o w}$.

Alternatively, for WEKA 3.6.10, the user may download the configuration file at

"http://sites.labic.icmc.usp.br/igorab/gridSearchSMO . conf".

It can be loaded using the Open button on the configuration window of GridSearch. After that, it remains to set the options:

- YBASE to the value $\frac{1}{2 d}$, where $d$ is the number of features (excluding the class attribute) in the dataset of interest;

- XMin to the value $\log _{10} C_{l o w}$;

- XMax to the value $a+\log _{10} C_{\text {low }}$, where $a$ is the number of candidates that should be investigated beyond $C_{\text {low }}$.

\footnotetext{
${ }^{1}$ http://www.cs.waikato.ac.nz/ml/weka/
} 

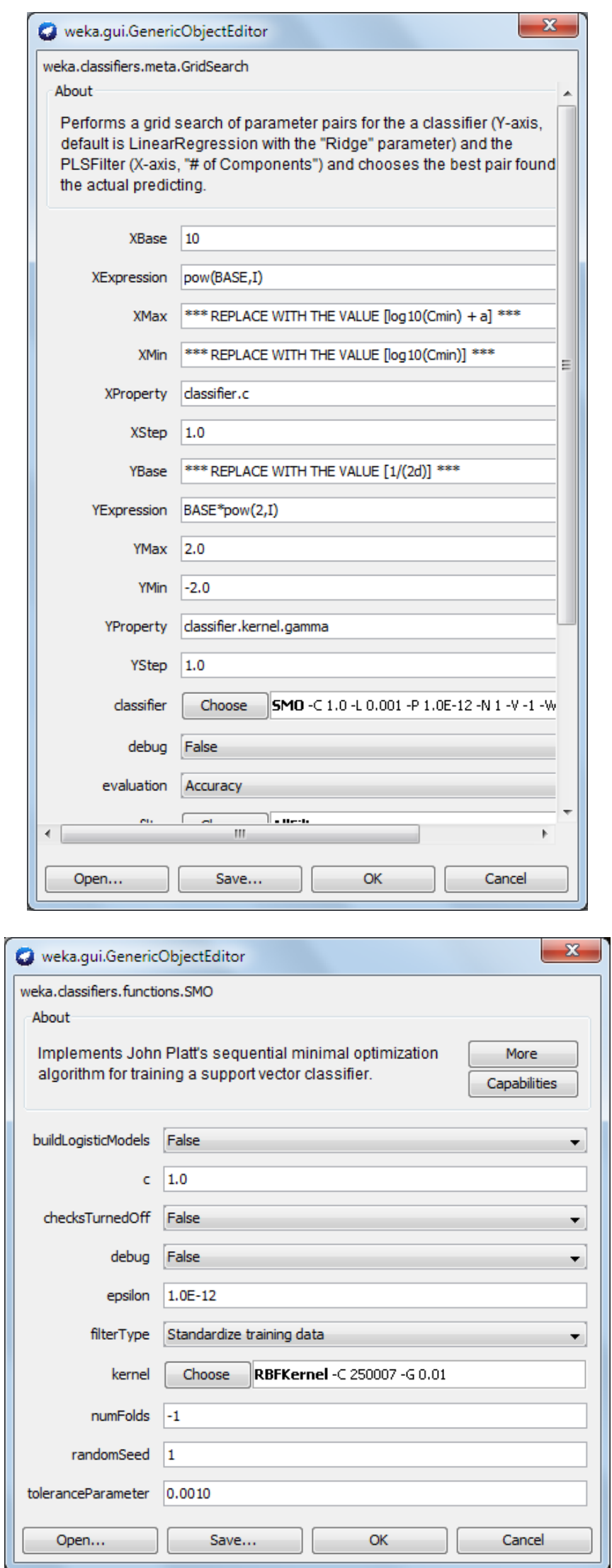

Figure A.1: Configuration options for instantiating the proposed procedure of SVMRBF parameter selection using WEKA's GridSearch meta-classifier. Note that both standardization and the RBF kernel should be enabled on the SMO configuration window. 


\section{A.2 Synthetic Models for the Mutual Information Ex- periments}

The following pictures are samples of the two-class data models described in Table 4.1.
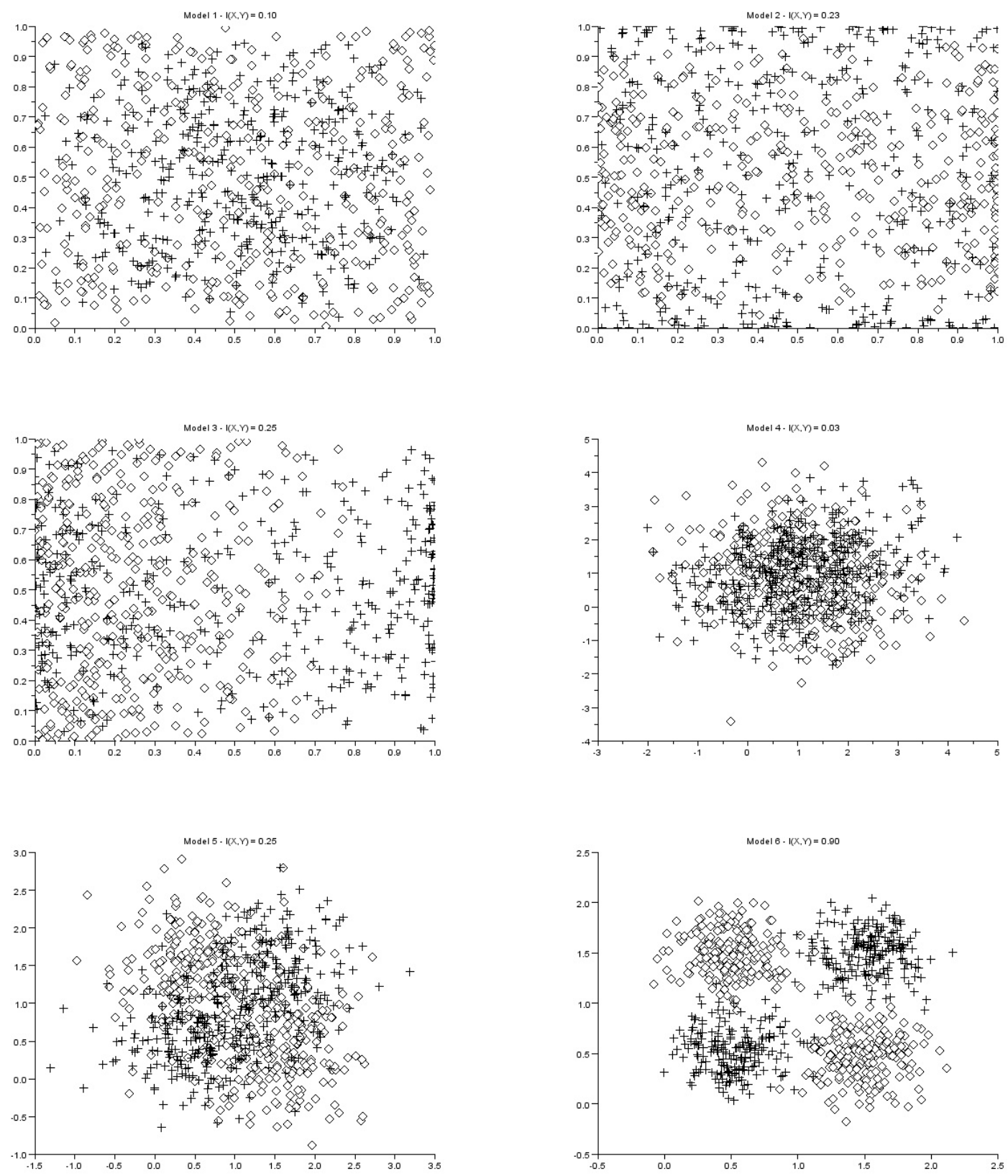

Figure A.2: Data models 1-6 

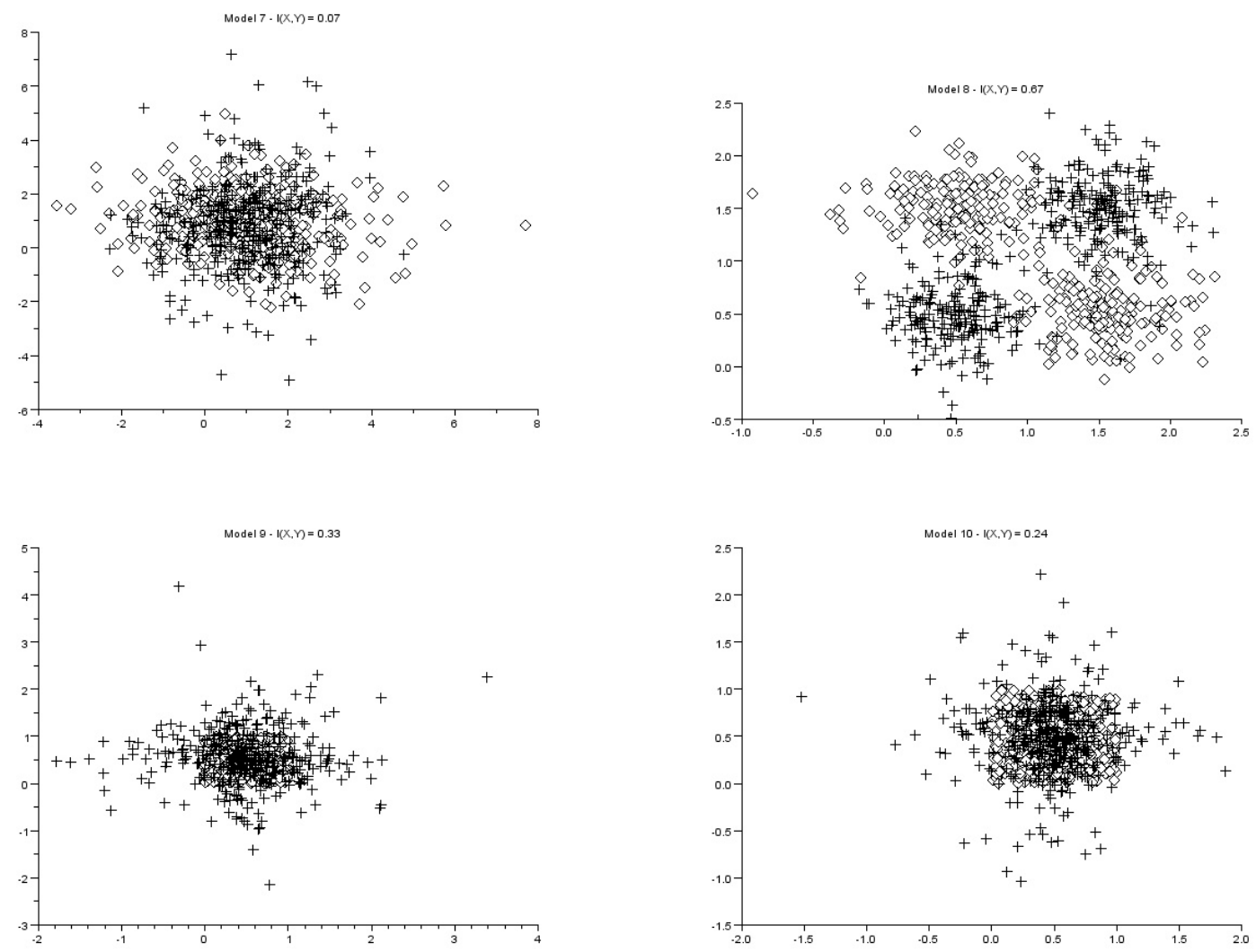

Figure A.3: Data models 7-10 


\section{References}

Akaike, H. (1970). Statistical predictor identification. Annals of The Institute of Statistical Mathematics, 22(1):203-217. Cited on page 110.

Alcalá-Fernández, J., Fernández, A., Luengo, J., Derrac, J., García, S., Sánchez, L., and Herrera, F. (2011). KEEL data-mining software tool: data set repository, integration of algorithms and experimental analysis framework. Journal of Multiple-Valued Logic and Soft Computing, 17(2-3):255-287. Cited on page 114.

Ben-Hur, A. and Weston, J. (2010). A user's guide to support vector machines. Methods in Molecular Biology, 609:223-239. Cited on page 82.

Bock, R. K., Chilingarian, A., Gaug, M., Hakl, F., Hengstebeck, T., Jirina, M., Klaschka, J., Kotrc, E., Savický, P., Towers, S., Vaiciulis, A., and Wittek, W. (2004). Methods for multidimensional event classification: a case study using images from a Cherenkov gamma-ray telescope. Nuclear Instruments and Methods in Physics Research A, 516:511-528. Cited on page 93.

Braga, I. (2013). The constructive density-ratio approach to mutual information estimation: An experimental comparison. In KDMile '13: Proceedings of 2013 Symposium on Knowledge Discovery, Mining and Learning, pages 1-4. Cited on page 27.

Braga, I. (2014). A constructive density-ratio approach to mutual information estimation: experiments in feature selection. Journal of Information and Data Management, 5(1):134-143. Cited on page 27.

Braga, I., do Carmo, L. P., Benatti, C. C., and Monard, M. C. (2013). A note on parameter selection for support vector machines. In MICAI '13: Proceedings of the 2013 Mexican International Conference on Artificial Intelligence, pages 233-244. Cited on pages 27 and 82 .

Braga, I. and Monard, M. C. (2013). Statistical and heuristic model selection in regularized least-squares. In BRACIS '13: Proceedings of the 2013 Brazilian Conference on Intelligent Systems, pages 231-236. Cited on page 27.

Braga, I. and Monard, M. C. (2015). Improving the kernel regularized least squares method for small-sample regression (in print). Neurocomputing. Cited on page 27. 
Bregman, L. (1967). The relaxation method of finding the common points of convex sets and its application to the solution of problems in convex programming. USSR Computational Mathematics and Mathematical Physics, 7(3):200-217. Cited on page 40.

Breiman, L. (1993). Hinging hyperplanes for regression, classification, and function approximation. IEEE Transactions on Information Theory, 39(3):999-1013. Cited on page 102 .

Brown, G., Pocock, A., Zhao, M.-J., and Luján, M. (2012). Conditional likelihood maximisation: A unifying framework for information theoretic feature selection. Journal of Machine Learning Research, 13(1):27-66. Cited on page 69.

Burges, C. J. C. (1998). A tutorial on support vector machines for pattern recognition. Data Mining and Knowledge Discovery, 2(2):121-167. Cited on page 80.

Chang, C.-C. and Lin, C.-J. (2011). LIBSVM: A library for support vector machines. ACM Transactions on Intelligent Systems and Technology, 2(3):27:1-27:27. Cited on page 82 .

Chapelle, O., Vapnik, V., and Bengio, Y. (2002). Model selection for small sample regression. Machine Learning, 48(1-3):9-23. Cited on pages 110, 114, and 122.

Cherkassky, V., Shao, X., Mulier, F., and Vapnik, V. (1999). Model complexity control for regression using VC generalization bounds. IEEE Transactions on Neural Networks, 10(5):1075-1089. Cited on pages 109, 114, and 122.

Cortes, C. and Vapnik, V. (1995). Support vector networks. Machine Learning, 20(3):273297. Cited on pages 25, 79, and 103.

Cortez, P., Cerdeira, A., Almeida, F., Matos, T., and Reis, J. (2009). Modeling wine preferences by data mining from physicochemical properties. Decision Support Systems, 47(4):547-553. Cited on page 115.

Cover, T. and Thomas, J. (2006). Elements of Information Theory. Wiley-Interscience. Cited on page 63.

Cristianini, N. and Shawe-Taylor, J. (2000). An Introduction to Support Vector Machines and Other Kernel-based Learning Methods. Cambridge University Press. Cited on page 80 .

Dvoretzky, A., Kiefer, J., and Wolfowitz, J. (1956). Asymptotic minimax character of the sample distribution function and of the classical multinomial estimator. The Annals of Mathematical Statistics, 27(3):642-669. Cited on page 34.

Elisseeff, A. and Pontil, M. (2002). Leave-one-out Error and Stability of Learning Algorithms with Applications. In Learning Theory and Practice. IOS Press. Cited on page 107 . 
Fornberg, B., Wright, G., and Larsson, E. (2004). Some observations regarding interpolants in the limit of flat radial basis functions. Computers $\&$ Mathematics with Applications, 47(1):37-55. Cited on page 88.

Friedman, J. (1991). Multivariate adaptative regression splines. Annals of Statistics, 19(1):1-141. Cited on page 115 .

Girosi, F. (1997). An equivalence between sparse approximation and support vector machines. Technical report, Massachusetts Institute of Technology. http://cbcl.mit. edu/publications/ai-publications/1500-1999/AIM-1606.ps. Cited on pages 25 and 103.

Gorman, R. P. and Sejnowski, T. J. (1988). Analysis of hidden units in a layered network trained to classify sonar targets. Artificial Intelligence in Medicine, 1(1):75-89. Cited on page 93.

Gretton, A., Smola, A., Huang, J., Schmittfull, M., Borgwardt, K., and Scholkopf, B. (2009). Covariate shift by kernel mean matching. In Dataset Shift in Machine Learning, pages 131-160. MIT Press. Cited on pages 25, 43, 54, 58, 59, and 125.

Guyon, I. and Elisseeff, A. (2003). An introduction to variable feature selection. Journal of Machine Learning Research, 3:1157-1182. Cited on pages 63 and 69.

Hastie, T. J. and Tibshirani, R. J. (1990). Generalized Additive Models. CRC Monographs on Statistics and Applied Probability. Chapman and Hall. Cited on page 110.

Hellman, M. E. and Raviv, J. (1970). Probability of error, equivocation, and the Chernoff bound. IEEE Transactions on Information Theory, 16(4):368-372. Cited on page 68 .

Hsu, C.-W., Chang, C.-C., and Lin, C.-J. (2003). A practical guide to support vector classification. Technical report, National Taiwan University. http://www.csie.ntu. edu.tw/ cjlin/papers/guide/guide.pdf. Cited on pages 86 and 97.

Hyvärinen, A. and Oja, E. (2000). Independent Component Analysis: algorithms and applications. Neural Networks, 13(4-5):411-430. Cited on page 63.

Ivanov, V. K. (1963). On ill-posed problems. Matematicheskii Sbornik, 61(2):211-223. (In Russian). Cited on page 37.

Izmailov, R., Vapnik, V., and Vashist, A. (2013). Multidimensional splines with infinite number of knots as SVM kernels. In IJCNN '13: Proceedings of the 2013 International Joint Conference on Neural Networks, pages 1-7. Cited on pages 58, 89, 90, 99, 102, 106, and 113.

Kanamori, T., Hido, S., and Sugiyama, M. (2009). A least-squares approach to direct importance estimation. Journal of Machine Learning Research, 10:1391-1445. Cited on pages $43,44,55,58,59$, and 125 . 
Kimeldorf, G. and Wahba, G. (1970). A correspondence between Bayesian estimation on stochastic processes and smoothing by splines. Annals of Mathematical Statistics, 41(2):495-502. Cited on pages 52, 54, and 104.

Kononenko, I. (1994). Estimating attributes: analysis and extensions of RELIEF. In ECML '94: Proceedings of the 1994 European Conference on Machine Learning, pages 171-182. Cited on pages 27, 72, and 125 .

Kraskov, A., Stögbauer, H., and Grassberger, P. (2004). Estimating mutual information. Physical Review E, 69(066138). Cited on pages 63, 65, and 125.

Kurgan, L., Cios, K., Tadeusiewicz, R., Ogiela, M., and Goodenday, L. (2001). Knowledge discovery approach to automated cardiac SPECT diagnosis. Artificial Intelligence in Medicine, 23(2):149-169. Cited on page 93.

Little, M. A., McSharry, P. E., Roberts, S. J., Costello, D. A. E., and Moroz, I. M. (2007). Exploiting nonlinear recurrence and fractal scaling properties for voice disorder detection. BioMedical Engineering OnLine, 6:1-23. Cited on page 93.

Lucas, D. D., Klein, R., Tannahill, J., Ivanova, D., Brandon, S., Domyancic, D., and Zhang, Y. (2013). Failure analysis of parameter-induced simulation crashes in climate models. Geoscientific Model Development, 6(4):1157-1171. Cited on page 93.

Luntz, A. and Brailovsky, V. (1969). On estimation of characters obtained in statistical procedure of recognition. Technicheskaya Kibernetica, 3. Cited on page 107.

Maji, S., , Berg, A., and Malik, J. (2013). Classification using intersection kernel support vector machines is efficient. In CVPR '08: Proceedings of the 2008 IEEE Conference on Computer Vision and Pattern Recognition, pages 1-8. Cited on page 102.

Mansouri, K., Ringsted, T., Ballabio, D., Todeschini, R., and Consonni, V. (2013). Quantitative structure-activity relationship models for ready biodegradability of chemicals. Journal of Chemical Information and Modeling, 53(4):867-878. Cited on page 93.

Massart, P. (1990). The tight constant in the Dvoretzky-Kiefer-Wolfowitz inequality. The Annals of Probability, 18(3):1269-1283. Cited on page 34.

Moro, S., Laureano, R., and Cortez, P. (2011). Using data mining for bank direct marketing: An application of the CRISP-DM methodology. In ESM '11: Proceedings of the European Simulation and Modelling Conference, pages 117-121. Cited on page 93.

Nguyen, X., Wainwright, M., and Jordan, M. (2008). Estimating divergence functionals and the likelihood ratio by penalized convex risk minimization. In NIPS '0\%: Advances in Neural Information Processing Systems 20, pages 1-8. Cited on pages 43 and 44. 
Nguyen, X., Wainwright, M., and Jordan, M. (2010). Estimating divergence functionals and the likelihood ratio by convex risk minimization. IEEE Transactions on Information Theory, 56:5847-5861. Cited on pages 25 and 43.

Nogueira, B. M. (2009). Evaluation of unsupervised feature selection methods for text mining (in portuguese). Master's thesis, Instituto de Ciências Matemáticas e de Computação, Universidade de São Paulo. http://www.teses.usp.br/teses/ disponiveis/55/55134/tde-06052009-154832/. Cited on page 93.

Pang, B. and Lee, L. (2004). A sentimental education: sentiment analysis using subjectivity. In ACL '04: Proceedings of the 2014 Conference of the Association for Computational Linguistics, pages 271-278. Cited on page 93.

Rifkin, R. M. (2006). Everything Old is New Again: a Fresh Look at Historical Approaches in Machine Learning. PhD thesis, MIT-Sloan School of Management. Cited on pages 25, 103, and 107.

Robnik-Sikonja, M. and Kononenko, I. (2003). Theoretical and empirical analysis of ReliefF and RReliefF. Machine Learning, 53(1-2):23-69. Cited on page 72.

Schölkopf, B. and Smola, A. J. (2002). Learning With Kernels: Support Vector Machines, Regularization, Optimization and Beyond. MIT Press. Cited on page 103.

Schuurmans, D. (1997). A new metric-based approach to model selection. In $A A A I$ '9\%: Proceedings of the Fourteenth National Conference on Artificial Intelligence, pages 552-558. Cited on page 110 .

Schuurmans, D., Southey, F., Wilkinson, D., and Guo, Y. (2006). Metric-based approaches for semi-supervised regression and classification. In Semi-Supervised Learning, pages 421-451. MIT Press. Cited on pages 110, 114, and 122.

Schwarz, G. (1978). Estimating the dimension of a model. The Annals of Statistics, 6(2):461-464. Cited on page 110.

Shibata, R. (1981). An optimal selection of regression variables. Biometrika, 68(1):45-54. Cited on page 110 .

Shiryayev, A. N. (1984). Probability. Springer Science+Bussiness Media. Cited on pages 31 and 32.

Sigillito, V. G., Wing, S. P., Hutton, L. V., and Baker, K. B. (1989). Classification of radar returns from the ionosphere using neural networks. Technical Report 10, Johns Hopkins Applied Physics Laboratory. Cited on page 93.

Silverman, B. W. (1978). Density ratios, empirical likelihood and cot death. Journal of the Royal Statistical Society, Series C (Applied Statistics), 27(1):26-33. Cited on page 44 . 
Stefanyuk, A. R. (1986). Estimation of the likelihood ratio function in the "disorder" problem of random processes. Automation and Remote Control, 9:53-59. Cited on pages 39 and 43 .

Steinwart, I. (2001). On the influence of the kernel on the consistency of support vector machines. Journal of Machine Learning Research, 2:67-93. Cited on pages 86 and 112.

Stiglic, G. and Kokol, P. (2010). Stability of ranked gene lists in large microarray analysis studies. Journal of Biomedicine and Biotechnology, 2010:1-9. Cited on page 93.

Street, N., Wolberg, W. H., and Mangasarian, O. L. (1993). Nuclear feature extraction for breast tumor diagnosis. In ISET/SPIE '93: Proceedings of the International Symposium on Electronic Imaging: Science and Technology, pages 861-870. Cited on page 93.

Stummer, W. (2007). Some Bregman distances between financial diffusion processes. Proceedings in Applied Mathematics and Mechanics, 7(1):503-504. Cited on page 41.

Sugiyama, M., Nakajima, S., Kashima, H., von Bünau, P., and Kawanabe, M. (2008). Direct importance estimation with model selection and its application to covariate shift adaptation. In NIPS '0\%: Advances in Neural Information Processing Systems 20, pages $1-8$. Cited on pages $43,55,58,66$, and 125 .

Sugiyama, M., Suzuki, T., and Kanamori, T. (2011). Density Ratio Estimation in Machine Learning. Cambridge University Press. Cited on pages 24, 25, and 63.

Sugiyama, M., Suzuki, T., and Kanamori, T. (2012). Density-ratio matching under the Bregman divergence: A unified framework of density-ratio estimation. Annals of the Institute of Statistical Mathematics, 64(5):1009-1044. Cited on pages 31, 40, and 42.

Suzuki, T., Sugiyama, M., and Tanaka, T. (2009). Mutual information approximation via maximum likelihood estimation of density ratio. In ISIT '09: Proceedings of the 2009 IEEE International Symposium on Information Theory, pages 463-467. Cited on pages $63,65,71$, and 125 .

Tikhonov, A. (1963). Solution of incorrectly formulated problems and the regularization method. Soviet Mathematics - Doklady, 4:1035-1038. (In Russian). Cited on pages 37 and 39 .

van Hulle, M. M. (2005). Edgeworth approximation of multivariate differential entropy. Neural Computation, 17(9):1903-1910. Cited on pages 63, 65, and 125.

Vapnik, V., Braga, I., and Izmailov, R. (2014). A constructive setting for the problem of density ratio estimation. In SDM '14: Proceedings of the 2014 SIAM International Conference on Data Mining, pages 434-442. Cited on pages 27, 63, and 66.

Vapnik, V. N. (1998). Statistical Learning Theory. Wiley-Interscience. Cited on pages 24, 31, 32, 34, 35, 36, 38, 39, 43, 79, 80, 81, 83, 106, 109, 110, and 113. 
Vapnik, V. N. and Stefanyuk, A. R. (1978). Nonparametric methods for estimating probability densities. Automation and Remote Control, 8:38-52. Cited on page 38.

Villalobos, M. and Wahba, G. (1987). Inequality-constrained multivariate smoothing splines with application to the estimation of posterior probabilities. Journal of the American Statistical Association, 82(397):239-248. Cited on page 43.

Wahba, G. (1990). Spline models for observational data. Society for Industrial and Applied Mathematics (SIAM). Cited on pages 25, 103, 107, and 113.

Wahba, G. and Craven, P. (1979). Smoothing noisy data with spline functions: Estimating the correct degree of smoothing by the method of generalized cross-validation. Numerische Mathematik, 31:377-404. Cited on page 110.

Witten, I. H., Eibe, F., and Hall, M. H. (2010). Data Mining: Practical Machine Learning Tools and Techniques. Morgan Kaufmann Publishers Inc., third edition. Cited on page 82 .

Wolkowicz, H. and Styan, G. P. H. (1980). Bounds for eigenvalues using traces. Linear Algebra and its Applications, 29:471-506. Cited on page 106.

Yang, H. H. and Moody, J. (1999). Data visualization and feature selection: new algorithms for nongaussian data. In NIPS '99: Advances in Neural Information Processing Systems, pages 687-693. Cited on page 69.

Yeh, I.-C. (1998). Modeling of strength of high performance concrete using artificial neural networks. Cement and Concrete Research, 28(12):1797-1808. Cited on page 115. 